\title{
ON THE LOCAL LANGLANDS CORRESPONDENCE AND ARTHUR CONJECTURE FOR EVEN ORTHOGONAL GROUPS
}

\author{
HIRAKU ATOBE AND WEE TECK GAN
}

\begin{abstract}
In this paper, we highlight and state precisely the local Langlands correspondence for quasi-split $\mathrm{O}_{2 n}$ established by Arthur. We give two applications: Prasad's conjecture and Gross-Prasad conjecture for $\mathrm{O}_{n}$. Also, we discuss the Arthur conjecture for $\mathrm{O}_{2 n}$, and establish the Arthur multiplicity formula for $\mathrm{O}_{2 n}$.
\end{abstract}

\section{Contents}

1. Introduction

2. Orthogonal groups

3. Local Langlands correspondence 362

4. Prasad's conjecture 378

5. Gross-Prasad conjecture 383

6. Arthur's multiplicity formula for $\mathrm{SO}\left(V_{2 n}\right) \quad 392$

7. Arthur's multiplicity formula for $\mathrm{O}\left(V_{2 n}\right) \quad 403$

Acknowledgments

References

\section{INTRODUCTION}

In his long-awaited book $\mathrm{Ar}$, Arthur obtained a classification of irreducible representations of quasi-split symplectic and special orthogonal groups over local fields of characteristic 0 (the local Langlands correspondence LLC) as well as a description of the automorphic discrete spectrum of these groups over number fields (the Arthur conjecture). He proved these results by establishing the twisted endoscopic transfer of automorphic representations from these classical groups to $\mathrm{GL}_{N}$ by exploiting the stabilization of the twisted trace formula of $\mathrm{GL}_{N}$ (which has now been completed by Waldspurger and Mœglin). However, for the quasi-split special even orthogonal groups $\mathrm{SO}_{2 n}$, the results are not as definitive as one hopes. More precisely, for a $p$-adic field $F$, Arthur only gave a classification of the irreducible representations of $\mathrm{SO}_{2 n}(F)$ up to conjugacy by $\mathrm{O}_{2 n}(F)$, instead of by $\mathrm{SO}_{2 n}(F)$. Likewise, over a number field $\mathbb{F}$, he does not distinguish between a square-integrable automorphic representation $\pi$ and its twist by the outer automorphism corresponding to an element of $\mathrm{O}_{2 n}(\mathbb{F}) \backslash \mathrm{SO}_{2 n}(\mathbb{F})$.

Received by the editors February 28, 2016, and, in revised form, March 5, 2017.

2010 Mathematics Subject Classification. Primary 11F70, 11R39. 
The reason for this less-than-optimal result for $\mathrm{SO}_{2 n}$ is that, for the purpose of the twisted endoscopic transfer, it is more natural to work with the orthogonal groups $\mathrm{O}_{2 n}$ instead of $\mathrm{SO}_{2 n}$. In fact, Arthur has obtained in $\mathrm{Ar}$, Theorems 2.2.1, 2.2.4] a full classification of the irreducible representations of $\mathrm{O}_{2 n}(F)$. It is from this that he deduced the weak LLC for $\mathrm{SO}_{2 n}(F)$ alluded to above. Indeed, the weak LLC for $\mathrm{SO}_{2 n}(F)$ is equivalent to the classification of irreducible representations of $\mathrm{O}_{2 n}(F)$ modulo twisting by the determinant character det.

Unfortunately, this rather complete result for $\mathrm{O}_{2 n}(F)$ was not highlighted in $\mathrm{Ar}$. One possible reason is that $\mathrm{O}_{2 n}$ is a disconnected linear algebraic group and so does not fit in the framework of the classical Langlands program; for example, one does not have a systematic definition of the $L$-group of a disconnected reductive group and so one does not have the notion of Langlands parameters. In choosing to stick to the context of the Langlands program, Arthur has highlighted the results for $\mathrm{SO}_{2 n}$ instead. However, it has been observed that a suitable $L$-group for $\mathrm{O}_{2 n}$ is the group $\mathrm{O}_{2 n}(\mathbb{C})$ and an $L$-parameter for $\mathrm{O}_{2 n}(F)$ should be an orthogonal representation of the Weil-Deligne group $W D_{F}$. A precise statement to this effect seems to be first formulated in the paper $[\mathrm{P} 2]$ of $\mathrm{D}$. Prasad.

The main goal of this paper is to highlight and state precisely the local Langlands correspondence for quasi-split $\mathrm{O}_{2 n}(F)$ established by Arthur in $\mathrm{Ar}$ using this notion of $L$-parameters, and to establish various desiderata of this LLC. We also formulate the natural extension of the LLC to the pure inner forms (using Vogan $L$-packets [V]). The statements can be found in Desiderata 3.9 and 3.12, We especially note the key role played by the local intertwining relation in Hypothesis 3.13. This local intertwining relation was established in $\mathrm{Ar}$ for quasi-split groups but is conjectural for pure inner forms.

Our main motivation for formulating a precise LLC for $\mathrm{O}_{2 n}$ is that the representations of $\mathrm{O}_{2 n}(F)$ arise naturally in various context, such as in the theory of theta correspondence. If one wants to describe the local theta correspondence for the dual pair $\mathrm{O}_{2 n}(F) \times \mathrm{Sp}_{2 m}(F)$, one would need a classification of irreducible representations of $\mathrm{O}_{2 n}(F)$. Thus, this paper lays the groundwork needed for our paper AG in which we determine the local theta liftings of tempered representations in terms of the local Langlands correspondence. Having described the LLC for $\mathrm{O}_{2 n}$, we give two applications:

- (Prasad's conjecture) We complete the results in the first author's paper At1, in which the local theta correspondences for the almost equal rank dual pairs $\mathrm{O}_{2 n}(F) \times \operatorname{Sp}_{2 n}(F)$ and $\mathrm{O}_{2 n}(F) \times \operatorname{Sp}_{2 n-2}(F)$ were determined in terms of the weak LLC for $\mathrm{O}_{2 n}(F)$. In particular, we describe these theta correspondences completely in terms of the LLC for $\mathrm{O}_{2 n}(F)$, thus completing the proof of Prasad's conjecture (Conjectures 4.2 and 4.6). The result is contained in Theorem 4.4 .

- (Gross-Prasad conjecture for $\mathrm{O}_{n}$ ) In At1, these theta correspondences were used to prove the Fourier-Jacobi case of the local Gross-Prasad (GP) conjecture for symplectic-metaplectic groups, by relating the Fourier-Jacobi case with the Bessel case of (GP) for $\mathrm{SO}_{n}$ (which has been established by Waldspurger). For this purpose, the weaker version of Prasad's conjecture (based on the weak LLC for $\mathrm{O}_{2 n}$ ) is sufficient. Now that we have the full Prasad's conjecture, we use the Fourier-Jacobi case of (GP) to prove a version of $(\mathrm{GP})$ for $\mathrm{O}_{n}$. In other words, we shall extend and establish the 
Gross-Prasad conjecture to the context of orthogonal groups (Conjecture 5.2). The result is contained in Theorem 5.6.

These results are related in a complicated manner. The following diagram is a summary of the situation:

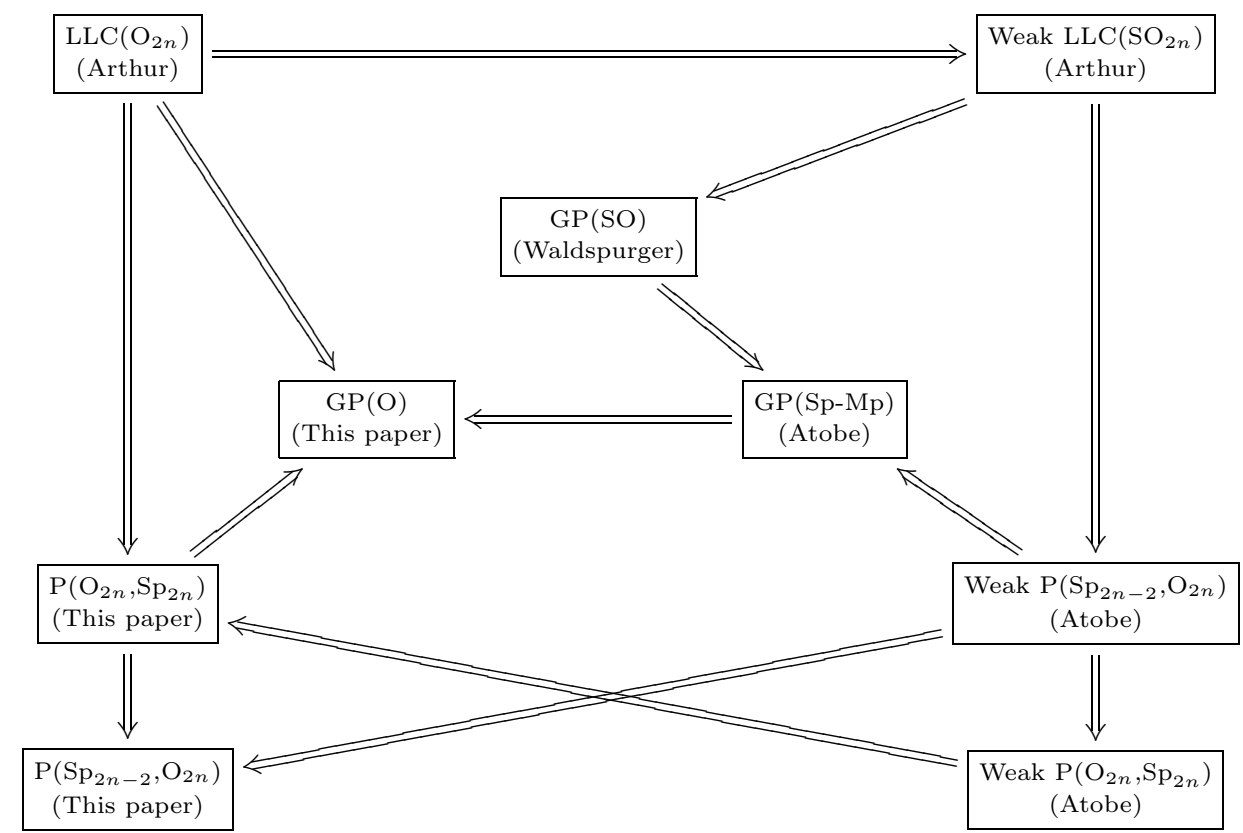

Here,

- $\operatorname{LLC}\left(\mathrm{O}_{2 n}\right)$ means the LLC for $\mathrm{O}_{2 n}$, which has been established by Arthur [Ar, Theorems 2.2.1, 2.2.4];

- Weak LLC $\left(\mathrm{SO}_{2 n}\right)$ means the weak LLC for $\mathrm{SO}_{2 n}$ or $\mathrm{O}_{2 n}$;

- Weak $\mathrm{P}\left(\mathrm{Sp}_{2 n-2}, \mathrm{O}_{2 n}\right)$ means the weaker version of Prasad's conjecture for $\left(\mathrm{Sp}_{2 n-2}, \mathrm{O}_{2 n}\right)$, which was proven in [At1, $\left.\$ 7\right]$;

- Weak $\mathrm{P}\left(\mathrm{O}_{2 n}, \mathrm{Sp}_{2 n}\right)$ means the weaker version of Prasad's conjecture for $\left(\mathrm{O}_{2 n}, \mathrm{Sp}_{2 n}\right)$, which follows from Weak $\mathrm{P}\left(\mathrm{Sp}_{2 n-2}, \mathrm{O}_{2 n}\right)$ (see [At1, §5.5]);

- $\mathrm{P}\left(\mathrm{O}_{2 n}, \mathrm{Sp}_{2 n}\right)$ means Prasad's conjecture for $\left(\mathrm{O}_{2 n}, \mathrm{Sp}_{2 n}\right)$ (Conjecture 4.2), which follows from Weak $\mathrm{P}\left(\mathrm{O}_{2 n}, \mathrm{Sp}_{2 n}\right)$ and Hypothesis 3.13 (see Theorem 4.4);

- $\mathrm{P}\left(\mathrm{Sp}_{2 n-2}, \mathrm{O}_{2 n}\right)$ means Prasad's conjecture for $\left(\mathrm{Sp}_{2 n-2}, \mathrm{O}_{2 n}\right)$ (Conjecture 4.6), which follows from Weak $\mathrm{P}\left(\mathrm{Sp}_{2 n-2}, \mathrm{O}_{2 n}\right)$ and $\mathrm{P}\left(\mathrm{O}_{2 n}, \mathrm{Sp}_{2 n}\right)$ (see Theorem 4.7);

- $\mathrm{GP}(\mathrm{SO})$ means the local Gross-Prasad (GP) conjecture for special orthogonal groups, which was established by Waldspurger [W2, W3, W5, W6];

- $\mathrm{GP}(\mathrm{Sp}-\mathrm{Mp})$ means the local Gross-Prasad (GP) conjecture for symplecticmetaplectic groups, which was proven in [At1, Theorem 1.3] by using $\mathrm{GP}(\mathrm{SO})$ and Weak $\mathrm{P}\left(\mathrm{Sp}_{2 n-2}, \mathrm{O}_{2 n}\right)$;

- GP(O) means the local Gross-Prasad (GP) conjecture for orthogonal groups (Conjecture 5.2), which follows from GP(Sp-Mp) and $\mathrm{P}\left(\mathrm{O}_{2 n}, \mathrm{Sp}_{2 n}\right)$ (see Theorem 5.6). 
Finally, we discuss how the Arthur conjecture for the automorphic discrete spectrum of $\mathrm{SO}_{2 n}$ over a number field $\mathbb{F}$ can be extended to an analogous statement for $\mathrm{O}_{2 n}$. This is implicitly in $\mathrm{Ar}$, but not precisely formulated. In particular, we describe the automorphic discrete spectrum of $\mathrm{O}_{2 n}$ in terms of local and global Arthur packets, and establish the Arthur multiplicity formula (Theorem 7.1). Also we show that the tempered part of the automorphic discrete spectrum of $\mathrm{O}_{2 n}$ has multiplicity 1 (Proposition 7.2). This should lay the groundwork for a more precise study of the global theta correspondence for symplectic-orthogonal dual pairs.

Notation. Let $F$ be a non-archimedean local field of characteristic 0 , o be the ring of integers of $F, \varpi$ be a uniformizer, $q$ be the number of elements in the residue class field $\mathfrak{o} / \varpi \mathfrak{o}$ and $|\cdot|_{F}$ be the normalized absolute value on $F$ so that $|\varpi|_{F}=q^{-1}$. We denote by $W_{F}$ and $W D_{F}=W_{F} \times \mathrm{SL}_{2}(\mathbb{C})$ the Weil and Weil-Deligne groups of $F$, respectively. Fix a non-trivial additive character $\psi$ of $F$. For $c \in F^{\times}$, we define an additive character $\psi_{c}$ or $c \psi$ of $F$ by

$$
\psi_{c}(x)=c \psi(x)=\psi(c x) .
$$

We set $\chi_{c}=(\cdot, c)$ to be the quadratic character of $F^{\times}$associated to $c \in F^{\times} / F^{\times 2}$. Here $(\cdot, \cdot)$ is the quadratic Hilbert symbol of $F$. For a totally disconnected locally compact group $G$, we denote the set of equivalence classes of irreducible smooth representations of $G$ by $\operatorname{Irr}(G)$. If $G$ is the group of $F$-points of a linear algebraic group over $F$, we denote by $\operatorname{Irr}_{\text {temp }}(G)\left(\operatorname{resp} \operatorname{Irr}_{\text {disc }}(G)\right)$ the subset of $\operatorname{Irr}(G)$ of classes of irreducible tempered representations (resp. irreducible discrete series representations). For a topological group $H$, we define the component group of $H$ by $\pi_{0}(H)=H / H^{\circ}$, where $H^{\circ}$ is the identity component of $H$. The Pontryagin dual (i.e., the character group) of a finite abelian group $A$ is denoted by $A^{D}$ or $\widehat{A}$.

\section{ORThOGONAL GROUPS}

In this section, we summarize facts about quasi-split orthogonal groups and their representations.

2.1. Orthogonal spaces. Let $V=V_{2 n}$ be a vector space of dimension $2 n$ over $F$ and

$$
\langle\cdot, \cdot\rangle_{V}: V \times V \rightarrow F
$$

be a non-degenerate symmetric bilinear form. We take a basis $\left\{e_{1}, \ldots, e_{2 n}\right\}$ of $V$, and define the discriminant of $V$ by

$$
\operatorname{disc}(V)=(-1)^{n} \operatorname{det}\left(\left(\left\langle e_{i}, e_{j}\right\rangle_{V}\right)_{i, j}\right) \bmod F^{\times 2} \in F^{\times} / F^{\times 2} .
$$

Let $\chi_{V}=(\cdot, \operatorname{disc}(V))$ be the character of $F^{\times}$associated with $F(\sqrt{\operatorname{disc}(V)})$. We call $\chi_{V}$ the discriminant character of $V$. The orthogonal group $\mathrm{O}(V)$ associated to $V$ is defined by

$$
\mathrm{O}(V)=\left\{g \in \mathrm{GL}(V) \mid\left\langle g v, g v^{\prime}\right\rangle_{V}=\left\langle v, v^{\prime}\right\rangle_{V} \text { for any } v, v^{\prime} \in V\right\} .
$$

Fix $c, d \in F^{\times}$. Let

$$
V_{(d, c)}=F[X] /\left(X^{2}-d\right)
$$

be a 2-dimensional vector space equipped with a bilinear form

$$
(\alpha, \beta) \mapsto\langle\alpha, \beta\rangle_{V_{(d, c)}}:=c \cdot \operatorname{tr}(\alpha \bar{\beta}),
$$


where $\beta \mapsto \bar{\beta}$ is the involution on $F[X] /\left(X^{2}-d\right)$ induced by $a+b X \mapsto a-b X$. This involution is regarded as an element $\epsilon \in \mathrm{O}\left(V_{(d, c)}\right)$. The images of $1, X \in F[X]$ in $V_{(d, c)}$ are denoted by $e, e^{\prime}$, respectively.

For $n>1$, we say that $V_{2 n}$ is associated to $(d, c)$ if

$$
V_{2 n} \cong V_{(d, c)} \oplus \mathbb{H}^{n-1}
$$

as orthogonal spaces, where $\mathbb{H}$ is the hyperbolic plane, i.e., $\mathbb{H}=F v_{i}+F v_{i}^{*}$ with $\left\langle v_{i}, v_{i}\right\rangle=\left\langle v_{i}^{*}, v_{i}^{*}\right\rangle=0$ and $\left\langle v_{i}, v_{i}^{*}\right\rangle=1$. Note that $\operatorname{disc}\left(V_{2 n}\right)=d \bmod F^{\times 2}$. The orthogonal group $\mathrm{O}\left(V_{2 n}\right)$ is quasi-split, and any quasi-split orthogonal group can be obtained in this way. Note that $V_{2 n}=V_{(d, c)} \oplus \mathbb{H}^{n-1} \cong V_{2 n}^{\prime}=V_{\left(d^{\prime}, c^{\prime}\right)} \oplus \mathbb{H}^{n-1}$ as orthogonal spaces if and only if $d \equiv d^{\prime} \bmod F^{\times 2}$ so that $E:=F(\sqrt{d})=F\left(\sqrt{d^{\prime}}\right)$ and $c \equiv c^{\prime} \bmod N_{E / F}\left(E^{\times}\right)$.

If we fix $(d, c)$, we set $V_{2 n}^{+}$to be the orthogonal space associated to $(d, c)$, and $V_{2 n}^{-}$to be an orthogonal space such that

$$
\operatorname{dim}\left(V_{2 n}^{+}\right)=\operatorname{dim}\left(V_{2 n}^{-}\right)=2 n \quad \text { and } \operatorname{disc}\left(V_{2 n}^{+}\right)=\operatorname{disc}\left(V_{2 n}^{-}\right)=d
$$

but $V_{2 n}^{+} \nRightarrow V_{2 n}^{-}$as orthogonal spaces. Such $V_{2 n}^{-}$exists uniquely up to isomorphisms unless $n=1$ and $d \in F^{\times 2}$. When $d \notin F^{\times 2}$, it is given so that $V_{2 n}^{-}$is the orthogonal space associated to $\left(d, c^{\prime}\right)$ with $c^{\prime} / c \notin N_{E / F}\left(E^{\times}\right)$where $E=F(\sqrt{d})$. When $d \in F^{\times 2}$ and $n>1$, it is given by $V_{2 n}^{-}=D \oplus \mathbb{H}^{n-2}$, where $D$ is a (unique up to isomorphism) division algebra over $F$ of dimension 4 , which is regarded as an orthogonal space with a bilinear form

$$
(\alpha, \beta) \mapsto \tau\left(\alpha \beta^{\iota}\right),
$$

where $\tau$ is the reduced trace and $\beta \mapsto \beta^{\iota}$ is the main involution. In this case, $\mathrm{O}\left(V_{2 n}^{-}\right)$is not quasi-split.

Similarly, let $V_{2 n+1}$ be an orthogonal space of dimension $2 n+1$. Its discriminant is defined by

$$
\operatorname{disc}\left(V_{2 n+1}\right)=2^{-1}(-1)^{n} \operatorname{det}\left(\left(\left\langle e_{i}, e_{j}\right\rangle_{V}\right)_{i, j}\right) \bmod F^{\times 2} \in F^{\times} / F^{\times 2},
$$

where $\left\{e_{1}, \ldots, e_{2 n+1}\right\}$ is a basis of $V_{2 n+1}$. When $n \geq 1$, for each $d \in F^{\times} / F^{\times 2}$, there are exactly two isomorphism classes $V_{2 n+1}^{+}$and $V_{2 n+1}^{-}$of orthogonal spaces of dimension $2 n+1$ and discriminant $d$. They are given as follows: Let $V_{1}=F$ be the orthogonal space of dimension 1 with a bilinear form

$$
(\alpha, \beta) \mapsto 2 d \cdot \alpha \beta \text {. }
$$

Then $\operatorname{disc}\left(V_{1}\right)=d$. For $n \geq 1$, we set $V_{2 n+1}^{+}=V_{1} \oplus \mathbb{H}^{n}$. On the other hand, let $D^{0}$ be the subspace of $D$ consisting of reduced trace zero elements. We regard $D^{0}$ as an orthogonal space of dimension 3 with a bilinear form

$$
(\alpha, \beta) \mapsto d \cdot \tau\left(\alpha \beta^{\iota}\right) .
$$

For $n \geq 1$, we set $V_{2 n+1}^{-}=D^{0} \oplus \mathbb{H}^{n-1}$. Note that $\mathrm{O}\left(V_{2 n+1}^{+}\right)$is split but $\mathrm{O}\left(V_{2 n+1}^{-}\right)$ is not quasi-split.

2.2. Generic representations. Suppose that $n>1$ in this subsection. Let $V=$ $V_{2 n}$ be the orthogonal space associated to $(d, c)$. We set

$$
X_{k}=F v_{1}+\cdots+F v_{k} \quad \text { and } \quad X_{k}^{*}=F v_{1}^{*}+\cdots+F v_{k}^{*}
$$


for $1 \leq k \leq n-1$. We denote by $B_{0}=T U_{0}$ the $F$-rational Borel subgroup of $\mathrm{SO}\left(V_{2 n}\right)$ stabilizing the complete flag

$$
0 \subset\left\langle v_{1}\right\rangle \subset\left\langle v_{1}, v_{2}\right\rangle \subset \cdots \subset\left\langle v_{1}, \ldots, v_{n-1}\right\rangle=X_{n-1},
$$

where $T$ is the $F$-rational torus stabilizing the lines $F v_{i}$ for $i=1, \ldots, n-1$. We identify $\mathrm{O}\left(V_{(d, c)}\right)$ as the subgroup of $\mathrm{O}\left(V_{2 n}\right)$ which fixes $\mathbb{H}^{n-1}$. Via the canonical embedding $\mathrm{O}\left(V_{(d, c)}\right) \hookrightarrow \mathrm{O}\left(V_{2 n}\right)$, we regard $\epsilon$ as an element in $\mathrm{O}\left(V_{2 n}\right)$. Note that $\epsilon$ depends on $(d, c)$. We define a generic character $\mu_{c}$ of $U_{0}$ by

$$
\mu_{c}(u)=\psi\left(\left\langle u v_{2}, v_{1}^{*}\right\rangle_{V}+\cdots+\left\langle u v_{n}, v_{n-2}^{*}\right\rangle_{V}+\left\langle u e, v_{n-1}^{*}\right\rangle_{V}\right) .
$$

Note that $\epsilon$ normalizes $U_{0}$ and fixes $\mu_{c}$.

Put $E=F(\sqrt{d})$. If $c^{\prime} \in c N_{E / F}\left(E^{\times}\right)$, then we have an isomorphism $V_{2 n}=$ $V_{(d, c)} \oplus \mathbb{H}^{n-1} \rightarrow V_{2 n}^{\prime}=V_{\left(d, c^{\prime}\right)} \oplus \mathbb{H}^{n-1}$, and so that we obtain an isomorphism

$$
f: \mathrm{O}\left(V_{2 n}\right) \rightarrow \mathrm{O}\left(V_{2 n}^{\prime}\right) .
$$

Moreover, we can take an isomorphism $f: \mathrm{O}\left(V_{2 n}\right) \rightarrow \mathrm{O}\left(V_{2 n}^{\prime}\right)$ such that $f\left(B_{0}\right)=B_{0}^{\prime}$, $f(T)=T^{\prime}$ and $f\left(\mathrm{O}\left(V_{(d, c)}\right)\right)=\mathrm{O}\left(V_{\left(d, c^{\prime}\right)}\right)$, where $B_{0}^{\prime}=T^{\prime} U_{0}^{\prime}$ and $T^{\prime}$ are the Borel subgroup and maximal torus of $\operatorname{SO}\left(V_{2 n}^{\prime}\right)$, respectively, defined as above. Let $\mathcal{F}$ be the set of such isomorphisms. Then the group $T^{\prime} \rtimes\left\langle\epsilon^{\prime}\right\rangle \cong \mathrm{O}\left(V_{\left(d, c^{\prime}\right)}\right) \times\left(F^{\times}\right)^{n-1}$ acts on $\mathcal{F}$ by

$$
\left(t^{\prime} \cdot f\right)(g)=t^{\prime} f(g) t^{\prime-1}
$$

for $t^{\prime} \in T^{\prime} \rtimes\left\langle\epsilon^{\prime}\right\rangle$ and $g \in \mathrm{O}\left(V_{2 n}\right)$. Here, $\epsilon^{\prime} \in \mathrm{O}\left(V_{\left(d, c^{\prime}\right)}\right)$ is an analogue to $\epsilon \in \mathrm{O}\left(V_{(d, c)}\right)$. Since $n>1$, this action of $T^{\prime} \rtimes\left\langle\epsilon^{\prime}\right\rangle$ is transitive.

Choosing $f \in \mathcal{F}$, we regard $\mu_{c^{\prime}}$ as a generic character of $U_{0}$ by

$$
U_{0} \stackrel{f}{\rightarrow} U_{0}^{\prime} \stackrel{\mu_{c^{\prime}}}{\longrightarrow} \mathbb{C}^{\times} .
$$

Note that the $T$-orbit of $\mu_{c^{\prime}}$ is independent of the choice of $f$ since $\epsilon^{\prime}$ fixes $\mu_{c^{\prime}}$.

We consider a 4-tuple $\left(V, B_{0}, T, \mu\right)$, where

- $V=V_{2 n}$ is the orthogonal space associated to some $(d, c)$;

- $B_{0}$ is an $F$-rational Borel subgroup of $\mathrm{SO}(V)$;

- $T$ is a maximal $F$-torus contained in $B_{0}$;

- $\mu$ is a generic character of $U_{0}$, where $U_{0}$ is the unipotent radical of $B_{0}$.

We say that two tuples $\left(V, B_{0}, T, \mu\right)$ and $\left(V^{\prime}, B_{0}^{\prime}, T^{\prime}, \mu^{\prime}\right)$ are equivalent if the following conditions hold:

(1) there exists an isomorphism $V \rightarrow V^{\prime}$ as orthogonal spaces, which induces a group isomorphism $f: \mathrm{O}(V) \rightarrow \mathrm{O}\left(V^{\prime}\right)$;

(2) $f\left(B_{0}\right)=B_{0}^{\prime}$ and $f(T)=T^{\prime}$ (so that $\left.f\left(U_{0}\right)=U_{0}^{\prime}\right)$;

(3) there exists $t \in T$ such that $\mu^{\prime} \circ f=\mu \circ \operatorname{Int}(t)$.

Proposition 2.1. Fix $d \in F^{\times} / F^{\times 2}$. For $c \in F^{\times}$, we associate the 4-tuple $\left(V, B_{0}, T, \mu\right)$, where

- $V=V_{2 n}$ is the orthogonal space associated to $(d, c)$;

- $B_{0}$ and $T$ are as above;

- $\mu=\mu_{c}$.

Then the map $c \mapsto\left(V, B_{0}, T, \mu\right)$ gives a canonical bijection (not depending on $\psi$ ) $F^{\times} / F^{\times 2} \rightarrow\left\{\right.$ equivalence classes of tuples $\left(V, B_{0}, T, \mu\right)$ with $\left.\operatorname{disc}(V)=d\right\}$. 
Proof. Let $V=V_{2 n}$ be the orthogonal space associated to $(d, c)$. By [GGP, §12], the map $c^{\prime} \mapsto \mu_{c^{\prime}} \circ f$ for $f \in \mathcal{F}$ gives a well-defined bijection

$$
c N_{E / F}\left(E^{\times}\right) / F^{\times 2} \rightarrow\left\{T \text {-orbits of generic characters of } U_{0}\right\},
$$

where $E=F(\sqrt{d})$. For $c^{\prime} \in c N_{E / F}\left(E^{\times}\right)$, let $V^{\prime}=V_{2 n}^{\prime}$ be the orthogonal space associated to $\left(d, c^{\prime}\right)$. Then two tuples $\left(V, B_{0}, T, \mu_{c^{\prime}} \circ f\right)$ and $\left(V^{\prime}, B_{0}^{\prime}, T^{\prime}, \mu_{c^{\prime}}\right)$ are equivalent to each other. This implies that the map

$$
\left.F^{\times} \rightarrow \text { \{equivalence classes of tuples }\left(V, B_{0}, T, \mu\right) \text { with } \operatorname{disc}(V)=d\right\}
$$

is surjective. Also, we note that for a generic character $\mu$, two tuples $\left(V, B_{0}, T, \mu_{c}\right)$ and $\left(V, B_{0}, T, \mu\right)$ are equivalent if and only if $\mu=\mu_{c} \circ \operatorname{Int}(t)$ for some $t \in T$. This implies that the above map induces the bijection

$$
F^{\times} / F^{\times 2} \rightarrow\left\{\text { equivalence classes of tuples }\left(V, B_{0}, T, \mu\right) \text { with } \operatorname{disc}(V)=d\right\},
$$

as desired.

Remark 2.2. (1) Let $\left(V,\langle\cdot, \cdot\rangle_{V}\right)$ be the orthogonal space associated to $(d, c)$. Fix $a \in F^{\times}$. We define a new orthogonal $\left(V^{\prime},\langle\cdot, \cdot\rangle_{V^{\prime}}\right)$ by $V^{\prime}=V$ as vector spaces and by

$$
\langle x, y\rangle_{V^{\prime}}=a \cdot\langle x, y\rangle_{V} .
$$

Then $\left(V^{\prime},\langle\cdot, \cdot\rangle_{V^{\prime}}\right)$ is associated to $(d, a c)$. As a subgroup of $\mathrm{GL}(V)=$ $\mathrm{GL}\left(V^{\prime}\right)$, we have identifications

$$
\mathrm{O}(V)=\mathrm{O}\left(V^{\prime}\right) \text { and } \mathrm{SO}(V)=\mathrm{SO}\left(V^{\prime}\right) .
$$

These identifications preserve $F$-rational Borel subgroups and maximal $F$ tori. Moreover, the generic character $\mu_{c}$ of a maximal unipotent subgroup of $\mathrm{SO}(V)$ is transferred to $\mu_{a c}$.

(2) Let $V_{2 n+1}$ and $W_{2 n}$ be an orthogonal space and the symplectic space over $F$ of dimension $2 n+1$ and $2 n$, respectively. If the associated special orthogonal group $\mathrm{SO}\left(V_{2 n+1}\right)$ is (quasi-)split, there is a unique conjugacy class of a pair $(B, T, \mu)$ of an $F$-rational Borel subgroup $B$ of $G=\mathrm{SO}\left(V_{2 n+1}\right)$, a maximal $F$-torus $T$ contained in $B$, and a generic character of $U$, where $U$ is the unipotent radical of $B$. On the other hand, for $G=\operatorname{Sp}\left(W_{2 n}\right)$, which is a split group, there is a canonical bijection (depending on $\psi$ )

$$
F^{\times} / F^{\times 2} \rightarrow\{\text { conjugacy classes of a pair }(B, T, \mu)\} .
$$

For more precisions, see [GGP, $\S 12]$. We denote the image of $c \in F^{\times} / F^{\times 2}$ by $\left(B, T, \mu_{c}\right)$.

Since $\epsilon^{\prime}$ stabilizes $\mu_{c^{\prime}}$, we can extend $\mu_{c^{\prime}}$ to $U^{\prime}=U_{0}^{\prime} \rtimes\left\langle\epsilon^{\prime}\right\rangle$. There are exactly two such extensions $\mu_{c^{\prime}}^{ \pm}: U^{\prime} \rightarrow \mathbb{C}^{\times}$which are determined by

$$
\mu_{c^{\prime}}^{ \pm}\left(\epsilon^{\prime}\right)= \pm 1 \text {. }
$$

We say that an irreducible smooth representation $\sigma$ of $\mathrm{O}\left(V_{2 n}\right)$ is $\mu_{c^{\prime}}^{ \pm}$-generic if

$$
\operatorname{Hom}_{f^{-1}\left(U^{\prime}\right)}\left(\sigma, \mu_{c^{\prime}}^{ \pm}\right) \neq 0
$$

for some $f \in \mathcal{F}$. For $\sigma_{0} \in \operatorname{Irr}\left(\mathrm{SO}\left(V_{2 n}\right)\right)$, the $\mu_{c^{\prime}}$-genericity is defined similarly. The $\mu_{c^{\prime}}^{ \pm}$-genericity and the $\mu_{c^{\prime}}$-genericity are independent of the choice of $f$. Note that $f^{-1}\left(U_{0}^{\prime}\right)=U_{0}$ for $f \in \mathcal{F}$, and

$$
\operatorname{dim}_{\mathbb{C}}\left(\operatorname{Hom}_{U_{0}}\left(\sigma_{0}, \mu_{c^{\prime}}\right)\right) \leq 1
$$

for $\sigma_{0} \in \operatorname{Irr}\left(\mathrm{SO}\left(V_{2 n}\right)\right)$. 
Lemma 2.3. Let $\sigma_{0} \in \operatorname{Irr}\left(\mathrm{SO}\left(V_{2 n}\right)\right)$.

(1) Assume that $\sigma_{0}$ can be extended to $\mathrm{O}\left(V_{2 n}\right)$. Then there are exactly two such extensions. Moreover, the following are equivalent:

(A) $\sigma_{0}$ is $\mu_{c^{\prime}}$-generic;

(B) exactly one of the two extensions is $\mu_{c^{\prime}}^{+}$-generic but not $\mu_{c^{\prime}}^{-}$-generic, and the other is $\mu_{c^{\prime}}^{-}$-generic but not $\mu_{c^{\prime}}^{+}$-generic.

(2) Assume that $\sigma_{0}$ cannot be extended to $\mathrm{O}\left(V_{2 n}\right)$. Then $\sigma=\operatorname{Ind}_{\mathrm{SO}\left(V_{2 n}\right)}^{\mathrm{O}\left(V_{2 n}\right)}\left(\sigma_{0}\right)$ is irreducible. Moreover, the following are equivalent:

(A) $\sigma_{0}$ is $\mu_{c^{\prime}}$-generic;

(B) $\sigma$ is both $\mu_{c^{\prime}}^{+}$-generic and $\mu_{c^{\prime}}^{-}$-generic.

Proof. The first assertions of (1) and (2) follow from the Clifford theory. It is easy that (B) implies (A) in both (1) and (2).

We show (A) implies (B). Let $\sigma_{0} \in \operatorname{Irr}\left(\mathrm{SO}\left(V_{2 n}\right)\right)$ be a $\mu_{c^{\prime}}$-generic representation, i.e.,

$$
\operatorname{dim}_{\mathbb{C}}\left(\operatorname{Hom}_{U_{0}}\left(\sigma_{0}, \mu_{c^{\prime}} \circ f\right)\right)=1
$$

for some $f \in \mathcal{F}$. By the Frobenius reciprocity, we have

$$
\begin{aligned}
& \operatorname{Hom}_{f-1}\left(U^{\prime}\right)\left(\operatorname{Ind}_{\mathrm{SO}\left(V_{2 n}\right)}^{\mathrm{O}\left(V_{2 n}\right)}\left(\sigma_{0}\right), \mu_{c^{\prime}}^{ \pm} \circ f\right) \\
& \cong \operatorname{Hom}_{\mathrm{O}\left(V_{2 n}\right)}\left(\operatorname{Ind}_{\mathrm{SO}\left(V_{2 n}\right)}^{\mathrm{O}\left(V_{2 n}\right)}\left(\sigma_{0}\right), \operatorname{Ind}_{f^{-1}\left(U^{\prime}\right)}^{\mathrm{O}\left(V_{2 n}\right)}\left(\mu_{c^{\prime}}^{ \pm} \circ f\right)\right) \\
& \cong \operatorname{Hom}_{\mathrm{SO}\left(V_{2 n}\right)}\left(\sigma_{0}, \operatorname{Ind}_{f^{-1}\left(U^{\prime}\right)}^{\mathrm{O}\left(V_{2 n}\right)}\left(\mu_{c^{\prime}}^{ \pm} \circ f\right) \mid \operatorname{SO}\left(V_{2 n}\right)\right) \\
& \cong \operatorname{Hom}_{\mathrm{SO}\left(V_{2 n}\right)}\left(\sigma_{0}, \operatorname{Ind}_{U_{0}}^{\mathrm{SO}\left(V_{2 n}\right)}\left(\mu_{c^{\prime}} \circ f\right)\right) \cong \operatorname{Hom}_{U_{0}}\left(\sigma_{0}, \mu_{c^{\prime}} \circ f\right) .
\end{aligned}
$$

In particular, if $\sigma=\operatorname{Ind}_{\mathrm{SO}\left(V_{2 n}\right)}^{\mathrm{O}\left(V_{2 n}\right)}\left(\sigma_{0}\right)$ is irreducible, then $\sigma$ is both $\mu_{c^{\prime}}^{+}$-generic and $\mu_{c^{\prime}}^{-}$-generic. This shows that (A) implies (B) in (2). If $\operatorname{Ind}_{\mathrm{SO}\left(V_{2 n}\right)}^{\mathrm{O}\left(V_{2 n}\right)}\left(\sigma_{0}\right) \cong \sigma_{1} \oplus \sigma_{2}$, then

$$
\begin{aligned}
& \operatorname{Hom}_{U_{0}}\left(\operatorname{Ind}_{\mathrm{SO}\left(V_{2 n}\right)}^{\mathrm{O}\left(V_{2 n}\right)}\left(\sigma_{0}\right) \mid \mathrm{SO}\left(V_{2 n}\right), \mu_{c^{\prime}} \circ f\right) \\
& \cong \operatorname{Hom}_{U_{0}}\left(\sigma_{1} \mid \mathrm{SO}\left(V_{2 n}\right), \mu_{c^{\prime}} \circ f\right) \oplus \operatorname{Hom}_{U_{0}}\left(\sigma_{2} \mid \mathrm{SO}\left(V_{2 n}\right), \mu_{c^{\prime}} \circ f\right),
\end{aligned}
$$

and $\operatorname{Hom}_{U_{0}}\left(\sigma_{i} \mid \mathrm{SO}\left(V_{2 n}\right), \mu_{c^{\prime}} \circ f\right)$ is $f^{-1}\left(\epsilon^{\prime}\right)$-stable for $i=1,2$. Hence this subspace is an eigenspace of $f^{-1}\left(\epsilon^{\prime}\right)$. Since both \pm 1 are eigenvalues of $f^{-1}\left(\epsilon^{\prime}\right)$ in $\operatorname{Hom}_{U_{0}}\left(\operatorname{Ind}_{\mathrm{SO}\left(V_{2 n}\right)}^{\mathrm{O}\left(V_{2 n}\right)}\left(\sigma_{0}\right), \mu_{c^{\prime}} \circ f\right)$, exactly one of $\sigma_{1}$ and $\sigma_{2}$ is $\mu_{c^{\prime}}^{+}$-generic, and the other is $\mu_{c}^{-}$-generic. This shows that (A) implies (B) in (1).

2.3. Unramified representations. Let $V=V_{2 n}$ be the orthogonal space associated to $(d, c)$. We say that $\mathrm{O}\left(V_{2 n}\right)$ (or $\mathrm{SO}\left(V_{2 n}\right)$ ) is unramified if $c, d \in \mathfrak{o}^{\times}$. In this subsection, we assume this condition. Recall that $V_{2 n}$ has a decomposition

$$
V_{2 n}=F v_{1}+\ldots F v_{n-1}+V_{(d, c)}+F v_{1}^{*}+\cdots+F v_{n-1}^{*}
$$

with $V_{(d, c)}=F e+F e^{\prime}$. We set

$$
\begin{cases}v_{0}=\frac{e+u^{-1} e^{\prime}}{2}, v_{0}^{*}=\frac{e-u^{-1} e^{\prime}}{2 c} & \text { if } d=u^{2}, \\ \mathfrak{o}_{E} \text { to be the ring of integers of } E=F(\sqrt{d}) \cong V_{(d, c)} & \text { if } d \notin \mathfrak{o}^{\times 2} .\end{cases}
$$


Note that $\left\langle v_{0}, v_{0}\right\rangle_{V}=\left\langle v_{0}^{*}, v_{0}^{*}\right\rangle_{V}=0$ and $\left\langle v_{0}, v_{0}^{*}\right\rangle_{V}=1$. Let $L_{2 n}$ be the $\mathfrak{o}$-lattice of $V_{2 n}$ defined by

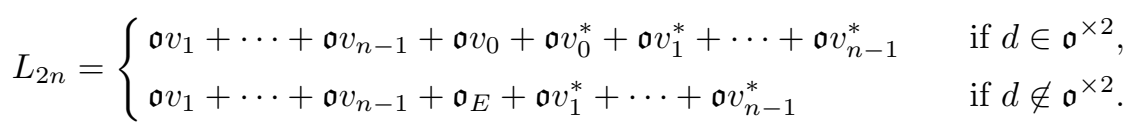

Let $K$ be the maximal compact subgroup of $\mathrm{O}\left(V_{2 n}\right)$ which preserves the lattice $L_{2 n}$. Note that $K$ contains $\epsilon$ and satisfies

$$
K=K_{0} \rtimes\langle\epsilon\rangle,
$$

where $K_{0}=K \cap \mathrm{SO}\left(V_{2 n}\right)$ is a maximal compact subgroup of $\mathrm{SO}\left(V_{2 n}\right)$.

Let $\sigma \in \operatorname{Irr}\left(\mathrm{O}\left(V_{2 n}\right)\right)$ and $\sigma_{0} \in \operatorname{Irr}\left(\mathrm{SO}\left(V_{2 n}\right)\right)$. We say that $\sigma$ (resp. $\left.\sigma_{0}\right)$ is unramified (with respect to $K$ (resp. $\left.K_{0}\right)$ ) if $\sigma$ (resp. $\sigma_{0}$ ) has a non-zero $K$-fixed (resp. $K_{0}$-fixed) vector. In this case, it is known that $\operatorname{dim}\left(\sigma^{K}\right)=\operatorname{dim}\left(\sigma_{0}^{K_{0}}\right)=1$.

Lemma 2.4. Let $\sigma_{0} \in \operatorname{Irr}\left(\mathrm{SO}\left(V_{2 n}\right)\right)$ be an unramified representation. Then $\operatorname{Ind}_{\mathrm{SO}\left(V_{2 n}\right)}^{\mathrm{O}\left(V_{2 n}\right)}\left(\sigma_{0}\right)$ has a unique irreducible unramified constituent.

Proof. Note that the map

$$
\operatorname{Ind}_{\mathrm{SO}\left(V_{2 n}\right)}^{\mathrm{O}\left(V_{2 n}\right)}\left(\sigma_{0}\right)^{K} \rightarrow \sigma_{0}^{K_{0}}, f \mapsto f(1)
$$

is a $\mathbb{C}$-linear isomorphism. Hence the assertion holds if $\operatorname{Ind}_{\mathrm{SO}\left(V_{2 n}\right)}^{\mathrm{O}\left(V_{2 n}\right)}\left(\sigma_{0}\right)$ is irreducible. Now suppose that $\operatorname{Ind}_{\mathrm{SO}\left(V_{2 n}\right)}^{\mathrm{O}\left(V_{2 n}\right)}\left(\sigma_{0}\right)$ is reducible. Then it decomposes into a direct sum

$$
\operatorname{Ind}_{\mathrm{SO}\left(V_{2 n}\right)}^{\mathrm{O}\left(V_{2 n}\right)}\left(\sigma_{0}\right) \cong \sigma_{1} \oplus \sigma_{2}
$$

We may assume that $\sigma_{1}$ and $\sigma_{2}$ are realized on the same space $\mathcal{V}$ as $\sigma_{0}$. Since $\sigma_{i}(\epsilon)$ preserve the one dimension subspace $\mathcal{V}^{K_{0}}$, we have $\sigma_{i}(\epsilon)= \pm$ id on $\mathcal{V}^{K_{0}}$. Since $\sigma_{1}(\epsilon)=-\sigma_{2}(\epsilon)$, exactly one $i \in\{1,2\}$ satisfies that $\sigma_{i}(\epsilon)=+1$. Then $\sigma_{i}$ is the unique irreducible unramified constituent of $\operatorname{Ind}_{\mathrm{SO}\left(V_{2 n}\right)}^{\mathrm{O}\left(V_{2 n}\right)}\left(\sigma_{0}\right)$.

\section{LOCAL LANGLANDS CORRESPONDENCE}

In this section, we explain the desideratum for the local Langlands correspondence for (special) orthogonal groups and symplectic groups.

3.1. Self-dual representations of $W D_{F}$ and its component groups. Let $M$ be a finite dimensional vector space over $\mathbb{C}$. We say that a homomorphism $\phi: W D_{F} \rightarrow \mathrm{GL}(M)$ is a representation of $W D_{F}=W_{F} \times \mathrm{SL}_{2}(\mathbb{C})$ if

- $\phi\left(\right.$ Frob $\left._{F}\right)$ is semi-simple, where Frob ${ }_{F}$ is a geometric Frobenius element in $W_{F}$;

- the restriction of $\phi$ to $W_{F}$ is smooth;

- the restriction of $\phi$ to $\mathrm{SL}_{2}(\mathbb{C})$ is algebraic.

We call $\phi$ tempered if the image of $W_{F}$ is bounded.

We say that $\phi$ is orthogonal (resp. symplectic) if there exists a non-degenerate bilinear form $B: M \times M \rightarrow \mathbb{C}$ such that

$$
\left\{\begin{array}{l}
B(\phi(w) x, \phi(w) y)=B(x, y), \\
B(y, x)=b \cdot B(x, y)
\end{array}\right.
$$

for $x, y \in M$ and $w \in W D_{F}$, where $b=+1$ (resp. $b=-1$ ). In this case, $\phi$ is selfdual, i.e., $\phi$ is equivalent to its contragredient $\phi^{\vee}$. More precisely, see [GGP, §3]. 
For an irreducible representation $\phi_{0}$ of $W D_{F}$, we denote the multiplicity of $\phi_{0}$ in $\phi$ by $m_{\phi}\left(\phi_{0}\right)$. Suppose that $\phi$ is orthogonal (resp. symplectic). We can decompose

$$
\phi=m_{1} \phi_{1}+\cdots+m_{s} \phi_{s}+\phi^{\prime}+\phi^{\prime \vee},
$$

where $\phi_{1}, \ldots, \phi_{s}$ are distinct irreducible orthogonal (resp. symplectic) representations of $W D_{F}, m_{i}=m_{\phi}\left(\phi_{i}\right)$, and $\phi^{\prime}$ is a sum of irreducible representations of $W D_{F}$ which are not orthogonal (resp. symplectic). We say that a representation $\phi$ is discrete if $m_{i}=1$ for any $i=1, \ldots, s$ and $\phi^{\prime}=0$, i.e., $\phi$ is a multiplicity-free sum of irreducible orthogonal (resp. symplectic) representations of $W D_{F}$.

For a representation $\phi$ of $W D_{F}$, the $L$-factor and the $\varepsilon$-factor associated to $\phi$, which are defined in [T], are denoted by $L(s, \phi)$ and $\varepsilon(s, \phi, \psi)$, respectively. If $(\phi, M)$ is an orthogonal (resp. symplectic) representation with a $W D_{F}$-invariant symmetric (resp. alternate) bilinear form $B$, then we define the adjoint $L$-function $L(s, \phi, \mathrm{Ad})$ associated to $\phi$ to be the $L$-function associated to

$$
\operatorname{Ad} \circ \phi: W D_{F} \rightarrow \operatorname{GL}(\operatorname{Lie}(\operatorname{Aut}(M, B))) \text {. }
$$

We say that $\phi$ is generic if $L(s, \phi, \mathrm{Ad})$ is regular at $s=1$. Since $B$ is symmetric (resp. alternate), we have $\mathrm{Ad} \circ \phi \cong \wedge^{2} \phi$ (resp. Ad $\left.\circ \phi \cong \operatorname{Sym}^{2} \phi\right)$. Hence the adjoint $L$-function $L(s, \phi, \mathrm{Ad})$ is equal to the exterior square $L$-function $L\left(s, \phi, \wedge^{2}\right)=$ $L\left(s, \wedge^{2} \phi\right)$ (resp. the symmetric square $L$-function $\left.L\left(s, \phi, \operatorname{Sym}^{2}\right)=L\left(s, \operatorname{Sym}^{2} \phi\right)\right)$.

Let $\phi$ be a representation of $W D_{F}=W_{F} \times \mathrm{SL}_{2}(\mathbb{C})$. We denote the inertia subgroup of $W_{F}$ by $I_{F}$. We say that $\phi$ is unramified if $\phi$ is trivial on $I_{F} \times \mathrm{SL}_{2}(\mathbb{C})$. In this case, $\phi$ is a direct sum of unramified characters of $W_{F}^{\mathrm{ab}} \cong F^{\times}$.

Let $(\phi, M)$ be an orthogonal (resp. symplectic) representation of $W D_{F}$ with an invariant bilinear form $B$. Set $C_{\phi}$ to be the centralizer of $\operatorname{Im}(\phi)$ in $\operatorname{Aut}(M, B) \cong$ $\mathrm{O}(\operatorname{dim}(M), \mathbb{C})(\operatorname{resp} . \operatorname{Aut}(M, B) \cong \operatorname{Sp}(\operatorname{dim}(M), \mathbb{C}))$. Namely, $C_{\phi}$ is the subgroup of $\mathrm{GL}(M)$ consisting of the elements $g \in \mathrm{GL}(M)$ such that $B(g x, g y)=B(x, y)$ for any $x, y \in M$, and $g \phi(w)=\phi(w) g$ for any $w \in W D_{F}$. Also we put

$$
C_{\phi}^{+}=C_{\phi} \cap \mathrm{SL}(M) .
$$

Finally, we define the large component group $A_{\phi}$ by

$$
A_{\phi}=\pi_{0}\left(C_{\phi}\right) .
$$

The image of $C_{\phi}^{+}$under the canonical map $C_{\phi} \rightarrow A_{\phi}$ is denoted by $A_{\phi}^{+}$, and called the component group of $\phi$. By GGP $\S 4], A_{\phi}$ and $A_{\phi}^{+}$are described explicitly as follows:

Let $\phi=m_{1} \phi_{1}+\cdots+m_{s} \phi_{s}+\phi^{\prime}+\phi^{\prime \vee}$ be the decomposition as above. Then we have

$$
A_{\phi}=\bigoplus_{i=1}^{s}(\mathbb{Z} / 2 \mathbb{Z}) a_{i} \cong(\mathbb{Z} / 2 \mathbb{Z})^{s} .
$$

Namely, $A_{\phi}$ is a free $\mathbb{Z} / 2 \mathbb{Z}$-module of rank $s$ and $\left\{a_{1}, \ldots, a_{s}\right\}$ is a basis of $A_{\phi}$ with $a_{i}$ associated to $\phi_{i}$. For $a=a_{i_{1}}+\cdots+a_{i_{k}} \in A_{\phi}$ with $1 \leq i_{1}<\cdots<i_{k} \leq s$, we put

$$
\phi^{a}=\phi_{i_{1}} \oplus \cdots \oplus \phi_{i_{k}} .
$$

Also, we put

$$
z_{\phi}:=\sum_{i=1}^{s} m_{\phi}\left(\phi_{i}\right) \cdot a_{i}=\sum_{i=1}^{s} m_{i} \cdot a_{i} \in A_{\phi} .
$$


This is the image of $-\mathbf{1}_{M} \in C_{\phi}$. We call $z_{\phi}$ the central element in $A_{\phi}$. The determinant map det: $\mathrm{GL}(M) \rightarrow \mathbb{C}^{\times}$gives a homomorphism

$$
\operatorname{det}: A_{\phi} \rightarrow \mathbb{Z} / 2 \mathbb{Z}, \quad \sum_{i=1}^{s} \varepsilon_{i} a_{i} \mapsto \sum_{i=1}^{s} \varepsilon_{i} \cdot \operatorname{dim}\left(\phi_{i}\right) \quad(\bmod 2),
$$

where $\varepsilon_{i} \in\{0,1\}=\mathbb{Z} / 2 \mathbb{Z}$. Then we have $A_{\phi}^{+}=\operatorname{ker}($ det $)$. Note that if $\phi$ is symplectic, then $A_{\phi}^{+}=A_{\phi}$.

By [GGP, §4], for each $c \in F^{\times}$, we can define a character $\eta_{\phi, c}$ of $A_{\phi}$ by

$$
\eta_{\phi, c}(a)=\operatorname{det}\left(\phi^{a}\right)(c) .
$$

Note that $\eta_{\phi, c}\left(z_{\phi}\right)=1$ if and only if $c \in \operatorname{ker}(\operatorname{det}(\phi))$.

3.2. $L$-groups and $L$-parameters. Let $V_{2 n}$ be the orthogonal space associated to $(d, c)$ for some $c, d \in F^{\times}$. We put $E=F(\sqrt{d})$. Then the Langlands dual group of $\mathrm{SO}\left(V_{2 n}\right)$ is the complex Lie group $\mathrm{SO}(2 n, \mathbb{C})$. We use

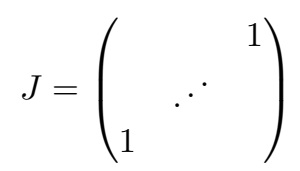

to define $\mathrm{O}(2 n, \mathbb{C})$, i.e., $\mathrm{O}(2 n, \mathbb{C})=\left\{g \in \mathrm{GL}_{2 n}(\mathbb{C}) \mid{ }^{t} g J g=J\right\}$. We denote the $L$-group of $\mathrm{SO}\left(V_{2 n}\right)$ by ${ }^{L}\left(\mathrm{SO}\left(V_{2 n}\right)\right)=\mathrm{SO}(2 n, \mathbb{C}) \rtimes W_{F}$. The action of $W_{F}$ on the dual group $\mathrm{SO}(2 n, \mathbb{C})$ factors through $W_{F} / W_{E} \cong \operatorname{Gal}(E / F)$. If $E \neq F$, i.e., $\mathrm{SO}\left(V_{2 n}\right)$ is not split, then the generator $\gamma \in \operatorname{Gal}(E / F)$ acts on $\mathrm{SO}(2 n, \mathbb{C})$ by the inner automorphism of

$$
\epsilon=\left(\begin{array}{llll}
\mathbf{1}_{n-1} & & & \\
& 0 & 1 & \\
& 1 & 0 & \\
& & & \mathbf{1}_{n-1}
\end{array}\right) \in \mathrm{O}(2 n, \mathbb{C}) .
$$

Hence by $\gamma \mapsto \epsilon$, we have the homomorphism

$$
{ }^{L}\left(\mathrm{SO}\left(V_{2 n}\right)\right)=\mathrm{SO}(2 n, \mathbb{C}) \rtimes W_{F} \rightarrow \mathrm{SO}(2 n, \mathbb{C}) \rtimes \mathrm{Gal}(E / F) \cong \mathrm{O}(2 n, \mathbb{C}) .
$$

On the other hand, if $E=F$, i.e., $\mathrm{SO}\left(V_{2 n}\right)$ is split, then $W_{F}$ acts on $\mathrm{SO}(2 n, \mathbb{C})$ trivially so that we have the homomorphism

$$
{ }^{L}\left(\mathrm{SO}\left(V_{2 n}\right)\right)=\mathrm{SO}(2 n, \mathbb{C}) \rtimes W_{F} \rightarrow \mathrm{SO}(2 n, \mathbb{C}) \hookrightarrow \mathrm{O}(2 n, \mathbb{C}) .
$$

We call the homomorphism

$$
{ }^{L}\left(\mathrm{SO}\left(V_{2 n}\right)\right) \rightarrow \mathrm{O}(2 n, \mathbb{C}) \hookrightarrow \mathrm{GL}_{2 n}(\mathbb{C})
$$

the standard representation of ${ }^{L}\left(\mathrm{SO}\left(V_{2 n}\right)\right)$.

Similarly, let $V_{2 n+1}$ (resp. $W_{2 n}$ ) be an orthogonal (resp. symplectic) space over $F$ of dimension $2 n+1$ (resp. $2 n$ ), and $\mathrm{SO}\left(V_{2 n+1}\right)$ (resp. $\operatorname{Sp}\left(W_{2 n}\right)$ ) be the associated special orthogonal (resp. symplectic) group. Its Langlands dual group is the complex Lie group $\operatorname{Sp}(2 n, \mathbb{C})$ (resp. $\mathrm{SO}(2 n+1, \mathbb{C})$ ), and its $L$-group is the direct product $\operatorname{Sp}(2 n, \mathbb{C}) \times W_{F}$ (resp. $\left.\mathrm{SO}(2 n+1, \mathbb{C}) \times W_{F}\right)$. In particular, the $L$-group has a standard representation ${ }^{L}\left(\mathrm{SO}\left(V_{2 n+1}\right)\right) \rightarrow \mathrm{Sp}(2 n, \mathbb{C}) \hookrightarrow \mathrm{GL}_{2 n}(\mathbb{C})$ $\left(\right.$ resp. $\left.{ }^{L}\left(\mathrm{Sp}\left(W_{2 n}\right)\right) \rightarrow \mathrm{SO}(2 n+1, \mathbb{C}) \hookrightarrow \mathrm{GL}_{2 n+1}(\mathbb{C})\right)$.

Let $G \in\left\{\mathrm{SO}\left(V_{2 n+1}\right), \mathrm{Sp}\left(W_{2 n}\right), \mathrm{SO}\left(V_{2 n}\right)\right\}$. Its Langlands dual group and $L$-group are denoted by $\widehat{G}$ and ${ }^{L} G$, respectively. As we have seen above, there is a standard 
representation ${ }^{L} G \rightarrow \mathrm{GL}_{N}(\mathbb{C})$ for some $N$. An admissible homomorphism of $G$ is a homomorphism

$$
\underline{\phi}: W D_{F} \rightarrow{ }^{L} G
$$

which commutes the projections $W D_{F} \rightarrow W_{F}$ and ${ }^{L} G \rightarrow W_{F}$. We put

$$
\Phi(G)=\{\widehat{G} \text {-conjugacy classes of admissible homomorphisms of } G\} .
$$

For an admissible homomorphism $\underline{\phi}: W D_{F} \rightarrow{ }^{L} G$, by composing with the standard representation ${ }^{L} G \rightarrow \mathrm{GL}_{N}(\mathbb{C})$, we obtain a representation

$$
\phi: W D_{F} \rightarrow \mathrm{GL}_{N}(\mathbb{C}) .
$$

This is orthogonal or symplectic. When $G=\operatorname{SO}\left(V_{2 n+1}\right)$ or $G=\operatorname{Sp}\left(W_{2 n}\right)$, we have $\operatorname{det}(\phi)=\mathbf{1}$, whereas, when $G=\mathrm{SO}\left(V_{2 n}\right)$, we have $\operatorname{det}(\phi)=\chi_{V}$, which is the discriminant character of $V_{2 n}$. The map $\underline{\phi} \mapsto \phi$ gives identifications

$$
\Phi(G)= \begin{cases}\left\{\phi: W D_{F} \rightarrow \mathrm{Sp}(2 n, \mathbb{C})\right\} /(\mathrm{Sp}(2 n, \mathbb{C}) \text {-conjugacy }) & \text { if } G=\mathrm{SO}\left(V_{2 n+1}\right), \\ \left\{\phi: W D_{F} \rightarrow \mathrm{SO}(2 n+1, \mathbb{C})\right\} /(\mathrm{SO}(2 n+1, \mathbb{C}) \text {-conjugacy }) & \text { if } G=\mathrm{Sp}\left(W_{2 n}\right), \\ \left\{\phi: W D_{F} \rightarrow \mathrm{O}(2 n, \mathbb{C}) \mid \operatorname{det}(\phi)=\chi_{V}\right\} /(\mathrm{SO}(2 n, \mathbb{C}) \text {-conjugacy }) & \text { if } G=\mathrm{SO}\left(V_{2 n}\right) .\end{cases}
$$

We denote the subset of $\Phi(G)$ consisting of tempered (resp. discrete, generic) representations by $\Phi_{\text {temp }}(G)$ (resp. $\Phi_{\text {disc }}(G), \Phi_{\text {gen }}(G)$ ). Then we have a sequence

$$
\Phi_{\text {disc }}(G) \subset \Phi_{\text {temp }}(G) \subset \Phi_{\text {gen }}(G) .
$$

When $G=\mathrm{SO}\left(V_{2 n}\right)$, we define $\Phi^{\epsilon}\left(\mathrm{SO}\left(V_{2 n}\right)\right)$ to be the subset of $\Phi\left(\mathrm{SO}\left(V_{2 n}\right)\right)$ consisting of $\phi$ which contains an irreducible orthogonal representation of $W D_{F}$ of odd dimension. We put $\Phi_{*}^{\epsilon}\left(\mathrm{SO}\left(V_{2 n}\right)\right)=\Phi^{\epsilon}\left(\mathrm{SO}\left(V_{2 n}\right)\right) \cap \Phi_{*}\left(\mathrm{SO}\left(V_{2 n}\right)\right)$ for $* \epsilon$ \{disc, temp, gen $\}$.

3.3. Desideratum. Fix $(d, c) \in\left(F^{\times}\right)^{2}$. As in 2.1 there are two spaces $V_{2 n}^{+}$and $V_{2 n}^{-}$(resp. $V_{2 n+1}^{+}$and $V_{2 n+1}^{-}$) of $2 n$ dimensional (resp. $2 n+1$ dimensional) orthogonal spaces unless $n=1$ and $d \in F^{\times 2}$ (resp. unless $n=0$ ). When $V=V_{m}$, we write $V^{+}=V_{m}^{+}$and $V^{-}=V_{m}^{-}$, and we set $G\left(V^{\delta}\right)=\mathrm{SO}\left(V_{m}^{\delta}\right)$. On the other hand, there is only one symplectic space $W_{2 n}$ of dimension $2 n$. When $V=W_{2 n}$, we write $V^{+}=V^{-}=W_{2 n}$, and we set $G\left(V^{+}\right)=G\left(V^{-}\right)=\operatorname{Sp}\left(W_{2 n}\right)$.

Now we describe the desiderata for the local Langlands correspondence for special orthogonal groups and symplectic groups.

Desideratum 3.1 (LLC for $G(V))$. Fix $(d, c) \in\left(F^{\times}\right)^{2}$, and let

$$
V \in\left\{V_{2 n}, V_{2 n+1}, W_{2 n}\right\} .
$$

(1) There exists a "canonical" surjection

$$
\bigsqcup_{\delta \in\{ \pm 1\}} \operatorname{Irr}\left(G\left(V^{\delta}\right)\right) \rightarrow \Phi(G(V))
$$

which is a finite-to-one map. For $\phi \in \Phi(G)$, we denote by $\Pi_{\phi}$ the inverse image of $\phi$ under this map, and call $\Pi_{\phi}$ the L-packet of $\phi$.

(2) For each $c^{\prime} \in F^{\times}$, there exists a "canonical" bijection

$$
\iota_{c^{\prime}}: \Pi_{\phi} \rightarrow \widehat{A_{\phi}^{+}} \text {. }
$$


(3) We have

$$
\bigsqcup_{\delta \in\{ \pm 1\}} \operatorname{Irr}_{*}\left(G\left(V^{\delta}\right)\right)=\bigsqcup_{\phi \in \Phi_{*}(G(V))} \Pi_{\phi}
$$

for $* \in\{$ temp, disc $\}$.

(4) When $V$ is orthogonal, $\sigma \in \Pi_{\phi}$ is a representation of $G\left(V^{+}\right)$if and only if

$$
\iota_{c^{\prime}}(\sigma)\left(z_{\phi}\right)= \begin{cases}1 & \text { if } G(V)=\mathrm{SO}\left(V_{2 n+1}\right), \\ \chi_{V}\left(c^{\prime} / c\right) & \text { if } G(V)=\mathrm{SO}\left(V_{2 n}\right) .\end{cases}
$$

(5) Assume that $\phi=\phi_{\tau}+\phi_{0}+\phi_{\tau}^{\vee}$, where $\phi_{0}$ is an element in $\Phi_{\text {temp }}\left(G\left(V_{0}\right)\right)$ and $\phi_{\tau}$ is an irreducible tempered representation of $W D_{F}$ which corresponds to $\tau \in \operatorname{Irr}_{\text {temp }}\left(\mathrm{GL}_{k}(F)\right)$ with $V=V_{0} \oplus \mathbb{H}^{k}$. Then $G\left(V^{\delta}\right)$ has a parabolic subgroup $P$ whose Levi subgroup $M_{P}$ is of the form $\mathrm{GL}_{k}(F) \times G\left(V_{0}^{\delta}\right)$. Moreover, for $\sigma_{0} \in \Pi_{\phi_{0}} \cap \operatorname{Irr}_{\text {temp }}\left(G\left(V_{0}^{\delta}\right)\right)$, the induced representation

$$
\operatorname{Ind}_{P}^{G\left(V^{\delta}\right)}\left(\tau \otimes \sigma_{0}\right)
$$

is a multiplicity-free direct sum of tempered representations of $G\left(V^{\delta}\right)$. The L-packet $\Pi_{\phi}$ is given by

$$
\Pi_{\phi}=\left\{\sigma \mid \sigma \subset \operatorname{Ind}_{P}^{\mathrm{SO}\left(V_{2 n}\right)}\left(\tau \otimes \sigma_{0}\right) \text { for some } \sigma_{0} \in \Pi_{\phi_{0}}\right\} .
$$

Moreover if $\sigma \subset \operatorname{Ind}_{P}^{G\left(V^{\delta}\right)}\left(\tau \otimes \sigma_{0}\right)$, then $\iota_{c^{\prime}}(\sigma) \mid A_{\phi_{0}}^{+}=\iota_{c^{\prime}}\left(\sigma_{0}\right)$ for $c^{\prime} \in F^{\times}$.

(6) Assume that

$\phi=\left(\phi_{\tau_{1}}|\cdot|{ }_{F}^{s_{1}}+\cdots+\phi_{\tau_{r}}|\cdot|_{F}^{s_{r}}\right)+\phi_{0}+\left(\phi_{\tau_{1}}|\cdot|{ }_{F}^{s_{1}}+\cdots+\phi_{\tau_{r}}|\cdot|{ }_{F}^{s_{r}}\right)^{\vee}$,

where $\phi_{0}$ is an element in $\Phi_{\text {temp }}\left(G\left(V_{0}\right)\right), \phi_{\tau_{i}}$ is an irreducible tempered representation of $W D_{F}$ which corresponds to $\tau_{i} \in \operatorname{Irr}_{\text {temp }}\left(\mathrm{GL}_{k_{i}}(F)\right)$ with

$$
V=V_{0} \oplus \mathbb{H}^{k_{1}} \oplus \cdots \oplus \mathbb{H}^{k_{r}}
$$

and $s_{i}$ is a real number with $s_{1} \geq \cdots \geq s_{r}>0$. Then $G\left(V^{\delta}\right)$ has a parabolic subgroup $P$ whose Levi subgroup $M_{P}$ is of the form $\mathrm{GL}_{k_{1}}(F) \times$ $\cdots \times \mathrm{GL}_{k_{r}}(F) \times G\left(V_{0}^{\delta}\right)$. The L-packet $\Pi_{\phi}$ consists of the unique irreducible quotients $\sigma$ of the standard modules

$$
\operatorname{Ind}_{P}^{G\left(V^{\delta}\right)}\left(\tau_{1}|\operatorname{det}|_{F}^{s_{1}} \otimes \cdots \otimes \tau_{r}|\operatorname{det}|_{F}^{s_{r}} \otimes \sigma_{0}\right),
$$

where $\sigma_{0}$ runs over elements of $\Pi_{\phi_{0}}$. Moreover $\iota_{c^{\prime}}(\sigma) \mid A_{\phi_{0}}^{+}=\iota_{c^{\prime}}\left(\sigma_{0}\right)$ for $c^{\prime} \in F^{\times}$.

In this paper, we take the position that the stabilization of the twisted trace formula used in $\mathrm{Ar}$ is complete. See also two books of Møglin-Waldspurger MW2] and papers of Chaudouard-Laumon CL1,CL2. Then the following theorem holds.

Theorem $3.2(\underline{\mathrm{Ar}}])$. The local Lagnlands correspondence for quasi-split $\mathrm{SO}\left(V_{2 n+1}\right)$ and $\operatorname{Sp}\left(W_{2 n}\right)$ holds. Namely, for $V=V^{+} \in\left\{V_{2 n+1}^{+}, W_{2 n}\right\}$, there exist a surjective map

$$
\operatorname{Irr}_{\text {temp }}\left(G\left(V^{+}\right)\right) \rightarrow \Phi_{\text {temp }}(G(V))
$$

with the inverse image $\Pi_{\phi}$ of $\phi \in \Phi_{\text {temp }}(G(V))$, and a bijection

$$
\iota_{c^{\prime}}: \Pi_{\phi} \rightarrow\left(A_{\phi}^{+} /\left\langle z_{\phi}\right\rangle \cap A_{\phi}^{+}\right)^{\wedge}
$$


for $c^{\prime} \in F^{\times}$satisfying Desideratum 3.1. Here, the "canonical" means that $\iota_{c^{\prime}}$ satisfies endoscopic character identities [Ar, Theorem 2.2.1] and local intertwining relations $\underline{\mathrm{Ar}}$, Theorem 2.4.1].

Remark 3.3. (1) When $G\left(V^{+}\right)=\mathrm{SO}\left(V_{2 n+1}^{+}\right)$, we see that $A_{\phi}^{+}=A_{\phi}$, so that $A_{\phi}^{+} /\left\langle z_{\phi}\right\rangle \cap A_{\phi}^{+}=A_{\phi} /\left\langle z_{\phi}\right\rangle$. On the other hand, when $G\left(V^{+}\right)=\operatorname{Sp}\left(W_{2 n}\right)$, we see that $z_{\phi} \notin A_{\phi}^{+}$and $A_{\phi}=A_{\phi}^{+} \oplus\left\langle z_{\phi}\right\rangle$. Hence $A_{\phi}^{+} /\left\langle z_{\phi}\right\rangle \cap A_{\phi}^{+}=A_{\phi}^{+} \cong$ $A_{\phi} /\left\langle z_{\phi}\right\rangle$. In any case, an image of $\iota_{c^{\prime}}$ can be regarded as a character of $A_{\phi}$.

(2) Mœglin [M2, §1.4, Theorem 1.4.1] and Mœglin-Renard [MR2 have partially extended Theorem 3.2 to pure inner forms, though we are not sure if all the statements of Desideratum 3.1 were verified in their works. In particular, we are not sure if these works are compatible with the general theory of T. Kaletha on the normalization of transfer factors for inner forms Ka2 , and if the local character relations of Arthur $\mathrm{Ar}$, Theorems 2.2.1, 2.2.4] were verified.

(3) Desideratum 3.1 (1) says that there is a surjection from a union of the sets of irreducible representations of several groups. In fact, as in Theorem 3.2 . this surjectivity is required when we restrict it on the set of irreducible representations of a quasi-split group.

There are some properties of $\Pi_{\phi}$.

Proposition 3.4. If $V=V^{+} \in\left\{V_{2 n+1}^{+}, W_{2 n}\right\}$, then the following hold:

(1) For $c_{1}, c_{2} \in F^{\times}$and $\sigma \in \Pi_{\phi}$, we have

$$
\iota_{c_{2}}(\sigma) \cdot \iota_{c_{1}}(\sigma)^{-1}= \begin{cases}1 & \text { if } G\left(V^{+}\right)=\mathrm{SO}\left(V_{2 n+1}^{+}\right), \\ \eta_{\phi, c_{2} / c_{1}} & \text { if } G\left(V^{+}\right)=\operatorname{Sp}\left(W_{2 n}\right),\end{cases}
$$

as a character of $A_{\phi}^{+}$.

(2) $\phi$ is generic, i.e., $L(s, \phi, \mathrm{Ad})$ is regular at $s=1$ if and only if $\Pi_{\phi}$ contains a $\mu$-generic representation $\sigma$ for each generic character $\mu$.

(3) Suppose that $\phi$ is generic. When $G\left(V^{+}\right)=\mathrm{SO}\left(V_{2 n+1}^{+}\right)$, then $\sigma \in \Pi_{\phi}$ is generic if and only if $\iota_{c^{\prime}}(\sigma)$ is the trivial representation of $A_{\phi}$ for any $c^{\prime} \in$ $F^{\times}$. When $G\left(V^{+}\right)=\operatorname{Sp}\left(W_{2 n}\right)$, then for each $c^{\prime} \in F^{\times}, \sigma \in \Pi_{\phi}$ is $\mu_{c^{\prime}}$ generic if and only if $\iota_{c^{\prime}}(\sigma)$ is the trivial representation of $A_{\phi}^{+}$.

(4) If both $G\left(V^{+}\right)$and $\phi$ are unramified, then $\Pi_{\phi}$ contains a unique unramified representation $\sigma$, and it corresponds to the trivial representation of $A_{\phi}^{+}$ under $\iota_{1}$.

Proof. (1) is given in [Ka1, Theorem 3.3]. (2) is a conjecture of Gross-Prasad and Rallis ([GP Conjecture 2.6]), and has been proven by Gan-Ichino ([GI2, Appen$\operatorname{dix} \mathrm{B}])$. For (3), it is shown in [Ar, Proposition 8.3.2 (a)] supplemented by some results of many others that the representation $\sigma$ corresponding to the trivial representation of $A_{\phi}^{+}$under $\iota_{c^{\prime}}$ is $\left(\mu_{c^{\prime}}\right)$ generic. A simple proof of the other direction is given by the first author [At2. Finally, (4) is proven by Mœglin [M1].

3.4. Local Langlands correspondence for $\mathrm{O}\left(V_{2 n+1}\right)$. In this subsection, we extend the local Langlands correspondence for $\mathrm{SO}\left(V_{2 n+1}\right)$ to the one for $\mathrm{O}\left(V_{2 n+1}\right)$.

Fix $d \in F^{\times}$. Let $V_{2 n+1}^{ \pm}$be the orthogonal space of dimension $2 n+1$, discriminant $d$, and such that $\mathrm{O}\left(V_{2 n+1}^{+}\right)$is split but $\mathrm{O}\left(V_{2 n+1}^{-}\right)$is not quasi-split. For $\delta \in\{ \pm 1\}$, 
note that $\mathrm{O}\left(V_{2 n+1}^{\delta}\right)$ is the direct product

$$
\mathrm{O}\left(V_{2 n+1}^{\delta}\right)=\mathrm{SO}\left(V_{2 n+1}^{\delta}\right) \times\{ \pm \mathbf{1}\}
$$

where $\pm \mathbf{1}= \pm \mathbf{1}_{V_{2 n+1}^{\delta}}$ is the central elements in $\mathrm{O}\left(V_{2 n+1}^{\delta}\right)$. Hence any

$$
\tau \in \operatorname{Irr}\left(\mathrm{O}\left(V_{2 n+1}^{\delta}\right)\right)
$$

is determined by its restriction $\tau \mid \mathrm{SO}\left(V_{2 n+1}^{\delta}\right) \in \operatorname{Irr}\left(\mathrm{SO}\left(V_{2 n+1}^{\delta}\right)\right)$ and its central character $\omega_{\tau} \in\{ \pm \mathbf{1}\}^{\Upsilon} \cong\{ \pm 1\}$. We define $\Phi\left(\mathrm{O}\left(V_{2 n+1}\right)\right)$ by

$$
\Phi\left(\mathrm{O}\left(V_{2 n+1}\right)\right):=\Phi\left(\mathrm{SO}\left(V_{2 n+1}\right)\right) \times\{ \pm 1\} .
$$

For $(\phi, b) \in \Phi\left(\mathrm{O}\left(V_{2 n+1}\right)\right)$, we put

$$
\Pi_{\phi, b}=\left\{\tau \in \operatorname{Irr}\left(\mathrm{O}\left(V_{2 n+1}^{\delta}\right)\right)|\tau| \mathrm{SO}\left(V_{2 n+1}^{\delta}\right) \in \Pi_{\phi}, \omega_{\tau}(-1)=b\right\} .
$$

Then we have canonical bijections

$$
\Pi_{\phi, b} \stackrel{\text { Res }}{\longrightarrow} \Pi_{\phi} \stackrel{\iota_{c}}{\longrightarrow} \widehat{A_{\phi}} .
$$

Since $\iota_{c}$ does not depend on $c \in F^{\times}$, we denote the composition map by $\iota$. Also we have

$$
\Pi_{\phi,-b}=\Pi_{\phi, b} \otimes \operatorname{det} .
$$

3.5. Local Langlands correspondence for $\mathrm{SO}\left(V_{2 n}\right)$. In the rest of this section, fix $(d, c) \in F^{\times 2}$, and assume that $V=V_{2 n}$ so that $V^{\delta}=V_{2 n}^{\delta}$ for each $\delta \in\{ \pm 1\}$.

Recall that $\Phi\left(\mathrm{SO}\left(V_{2 n}\right)\right)$ is taken up to not equivalence of representations but $\mathrm{SO}(2 n, \mathbb{C})$-conjugacy. Because of this difference, Desideratum 3.1 has not been established. Arthur $\mathrm{Ar}$ has established LLC for $\mathrm{O}\left(V_{2 n}\right)$, and deduced a weaker version of Desideratum 3.1 as follows.

We introduce an equivalence relation $\sim_{\epsilon}$ on $\operatorname{Irr}\left(\operatorname{SO}\left(V_{2 n}^{\delta}\right)\right)$ for $\delta \in\{ \pm 1\}$. Choose an element $\epsilon$ in $\mathrm{O}\left(V_{2 n}^{\delta}\right)$ such that $\operatorname{det}(\epsilon)=-1$. For $\sigma_{0} \in \operatorname{Irr}\left(\operatorname{SO}\left(V_{2 n}^{\delta}\right)\right)$, we define its conjugate $\sigma_{0}^{\epsilon}$ by $\sigma_{0}^{\epsilon}(g)=\sigma_{0}\left(\epsilon^{-1} g \epsilon\right)$. Then the equivalence relation $\sim_{\epsilon}$ on $\operatorname{Irr}\left(\mathrm{SO}\left(V_{2 n}^{\delta}\right)\right)$ is defined by

$$
\sigma_{0} \sim_{\epsilon} \sigma_{0}^{\epsilon}
$$

The canonical map $\operatorname{Irr}\left(\mathrm{SO}\left(V_{2 n}^{\delta}\right)\right) \rightarrow \operatorname{Irr}\left(\mathrm{SO}\left(V_{2 n}^{\delta}\right)\right) / \sim_{\epsilon}$ is denoted by $\sigma_{0} \mapsto\left[\sigma_{0}\right]$. We say that $\left[\sigma_{0}\right] \in \operatorname{Irr}\left(\operatorname{SO}\left(V_{2 n}^{\delta}\right)\right) / \sim_{\epsilon}$ is tempered (resp. discrete, $\mu_{c^{\prime}}$-generic, unramified) if so is some (and hence any) representative $\sigma_{0}$.

Also, we introduce an equivalence relation $\sim_{\epsilon}$ on $\Phi\left(\mathrm{SO}\left(V_{2 n}\right)\right)$. For $\phi, \phi^{\prime} \in$ $\Phi\left(\operatorname{SO}\left(V_{2 n}\right)\right)$, we write $\phi \sim_{\epsilon} \phi^{\prime}$ if $\phi$ is $\mathrm{O}(2 n, \mathbb{C})$-conjugate to $\phi^{\prime}$, i.e., $\phi$ is equivalent to $\phi^{\prime}$ as representations of $W D_{F}$. The equivalence class of $\phi$ is also denoted by $\phi$.

The desiderata for the weaker version of the local Langlands correspondence for $\mathrm{SO}\left(V_{2 n}\right)$ are described as follows:

Desideratum 3.5 (Weak LLC for $\mathrm{SO}\left(V_{2 n}\right)$ ). Let $V_{2 n}$ be the orthogonal space associated to $(d, c)$.

(1) There exists a "canonical" surjection

$$
\bigsqcup_{\delta \in\{ \pm 1\}} \operatorname{Irr}\left(\mathrm{SO}\left(V_{2 n}^{\delta}\right)\right) / \sim_{\epsilon} \rightarrow \Phi\left(\mathrm{SO}\left(V_{2 n}\right)\right) / \sim_{\epsilon} .
$$

For $\phi \in \Phi\left(\mathrm{SO}\left(V_{2 n}\right)\right) / \sim_{\epsilon}$, we denote by $\Pi_{\phi}^{0}$ the inverse image of $\phi$ under this map, and call $\Pi_{\phi}^{0}$ the L-packet of $\phi$. 
(2) For each $c^{\prime} \in F^{\times}$, there exists a "canonical" bijection

$$
\iota_{c^{\prime}}: \Pi_{\phi}^{0} \rightarrow \widehat{A_{\phi}^{+}} \text {. }
$$

(3) The L-packets $\Pi_{\phi}^{0}$ and the bijections $\iota_{c^{\prime}}$ satisfy analogues of Desideratum $3.1(3)-(6)$.

(4) The following are equivalent:

- $\phi \in \Phi^{\epsilon}\left(\mathrm{SO}\left(V_{2 n}\right)\right) / \sim_{\epsilon}$;

- some $\left[\sigma_{0}\right] \in \Pi_{\phi}^{0}$ satisfies $\sigma_{0}^{\epsilon} \cong \sigma_{0}$;

- all $\left[\sigma_{0}\right] \in \Pi_{\phi}^{0}$ satisfy $\sigma_{0}^{\epsilon} \cong \sigma_{0}$.

Here, $\Phi^{\epsilon}\left(\mathrm{SO}\left(V_{2 n}\right)\right) / \sim_{\epsilon}$ is the subset of $\Phi\left(\mathrm{SO}\left(V_{2 n}\right)\right) / \sim_{\epsilon}$ consisting of $\phi$ which contains an irreducible orthogonal representation of $W D_{F}$ of odd dimension.

As in the case of $\mathrm{SO}\left(V_{2 n+1}^{+}\right)$, Arthur $\mathrm{Ar}$ proved the quasi-split case.

Theorem 3.6 $([\overline{\mathrm{Ar}}])$. Let $V_{2 n}^{+}=V_{2 n}$ be the orthogonal space associated to $(d, c)$. Set $E=F(\sqrt{d})$. Then there exist a surjective map

$$
\operatorname{Irr}\left(\mathrm{SO}\left(V_{2 n}^{+}\right)\right) / \sim_{\epsilon} \rightarrow \Phi\left(\mathrm{SO}\left(V_{2 n}\right)\right) / \sim_{\epsilon}
$$

with the inverse image $\Pi_{\phi}^{0}$ of $\phi \in \Phi\left(\mathrm{SO}\left(V_{2 n}\right)\right)$, and a bijection

$$
\iota_{c^{\prime}}: \Pi_{\phi}^{0} \rightarrow\left(A_{\phi}^{+} /\left\langle z_{\phi}\right\rangle\right)^{\wedge}
$$

for $c^{\prime} \in c N_{E / F}\left(E^{\times}\right)$satisfying Desideratum 3.5. Here, the "canonical" means that $\iota_{c^{\prime}}$ satisfies endoscopic character identities [Ar, Theorem 2.2.1] and local intertwining relations [Ar, Theorem 2.4.1]. Moreover, $\iota_{c^{\prime}}$ does not depend on $\psi$.

Remark 3.7. If $d \notin F^{\times 2}$, then both $\mathrm{SO}\left(V_{2 n}^{+}\right)$and $\mathrm{SO}\left(V_{2 n}^{-}\right)$are quasi-split, so that we may define $L$-packets $\Pi_{\phi}^{0}$ and bijections

$$
\begin{aligned}
& \iota_{c_{1}}: \Pi_{\phi}^{0} \cap \operatorname{Irr}\left(\operatorname{SO}\left(V_{2 n}^{+}\right)\right) / \sim_{\epsilon} \rightarrow\left(A_{\phi}^{+} /\left\langle z_{\phi}\right\rangle\right), \\
& \iota_{c_{2}}: \Pi_{\phi}^{0} \cap \operatorname{Irr}\left(\operatorname{SO}\left(V_{2 n}^{-}\right)\right) / \sim_{\epsilon} \rightarrow\left(A_{\phi}^{+} /\left\langle z_{\phi}\right\rangle\right),
\end{aligned}
$$

for $c_{1}, c_{2} \in F^{\times}$with $c_{1} \in c N_{E / F}\left(E^{\times}\right)$and $c_{2} \notin c N_{E / F}\left(E^{\times}\right)$. We may define $\iota_{c_{2}}([\sigma])$ for $[\sigma] \in \operatorname{Irr}\left(\mathrm{SO}\left(V_{2 n}^{+}\right)\right) / \sim_{\epsilon}$ by

$$
\iota_{c_{2}}([\sigma]):=\iota_{c_{1}}([\sigma]) \otimes \eta_{\phi, c_{1} / c_{2}}
$$

and define $\iota_{c_{1}}\left(\left[\sigma^{\prime}\right]\right)$ for $\left[\sigma^{\prime}\right] \in \operatorname{Irr}\left(\operatorname{SO}\left(V_{2 n}^{-}\right)\right) / \sim_{\epsilon}$ similarly. Then the character relations and local intertwining relations would continue to hold after modifying the transfer factor and the normalization of intertwining operators. See also [KMSW] and [Ka2, §5.4].

Also, an analogue of Proposition 3.4 holds.

Proposition 3.8. Assume Weak LLC for $\mathrm{SO}\left(V_{2 n}\right)$ (Desideratum 3.5).

(1) For $c_{1}, c_{2} \in F^{\times}$, we have

$$
\iota_{c_{2}}\left(\left[\sigma_{0}\right]\right)=\iota_{c_{1}}\left(\left[\sigma_{0}\right]\right) \otimes \eta_{\phi, c_{2} / c_{1}}
$$

as a character of $A_{\phi}^{+}$.

(2) $\phi$ is generic, i.e., $L(s, \phi, \mathrm{Ad})$ is regular at $s=1$ if and only if $\Pi_{\phi}^{0}$ contains a $\mu_{c^{\prime}}$-generic class $\left[\sigma_{0}\right]$ for each $c^{\prime} \in F^{\times}$. Note that if $c^{\prime} \notin c N_{E / F}\left(E^{\times}\right)$, then $\sigma_{0} \in \operatorname{Irr}\left(\mathrm{SO}\left(V_{2 n}^{-}\right)\right)$. 
(3) If $\phi$ is generic, then for each $c^{\prime} \in F^{\times},\left[\sigma_{0}\right] \in \Pi_{\phi}^{0}$ is $\mu_{c^{\prime}}$-generic if and only if $\iota_{\mathcal{C}^{\prime}}\left(\left[\sigma_{0}\right]\right)$ is the trivial representation of $A_{\phi}^{+}$.

(4) If both $\mathrm{SO}\left(V_{2 n}\right)$ and $\phi$ are unramified, then $\Pi_{\phi}^{0}$ contains a unique unramified class $\left[\sigma_{0}\right]$, and it corresponds to the trivial representation of $A_{\phi}^{+}$under $\iota_{c}$.

In particular, these properties hold for the L-packets $\Pi_{\phi}^{0} \cap \operatorname{Irr}\left(\mathrm{SO}\left(V_{2 n}\right)\right) / \sim_{\epsilon}$ of quasi-split $\mathrm{SO}\left(V_{2 n}\right)$ and $c_{1}, c_{2}, c^{\prime} \in c N_{E / F}\left(E^{\times}\right)$unconditionally.

3.6. Local Langlands correspondence for $\mathrm{O}\left(V_{2 n}\right)$. Let $V_{2 n}=V_{2 n}^{+}$be the orthogonal space associated to $(d, c)$, and $\epsilon \in \mathrm{O}\left(V_{2 n}\right)$ be as in $\$ 2.1$. Put $\theta=\operatorname{Int}(\epsilon)$. It is an element in Aut $\left(\mathrm{SO}\left(V_{2 n}\right)\right)$. In $[\mathrm{Ar}$, Arthur has established the local Langlands correspondence not for $\mathrm{O}\left(V_{2 n}\right)$ but for

$$
\mathrm{SO}\left(V_{2 n}\right) \rtimes\langle\theta\rangle .
$$

As topological groups, $\mathrm{O}\left(V_{2 n}\right)$ and $\mathrm{SO}\left(V_{2 n}\right) \rtimes\langle\theta\rangle$ are isomorphic. However, the isomorphism is not canonical. Indeed, there are exactly two isomorphisms $\mathrm{O}\left(V_{2 n}\right) \cong$ $\mathrm{SO}\left(V_{2 n}\right) \rtimes\langle\theta\rangle$ which are identity on $\mathrm{SO}\left(V_{2 n}\right)$, and they are determined by $\pm \epsilon \leftrightarrow \theta$. We use the isomorphism such that $\epsilon \leftrightarrow \theta$. Via this isomorphism, we translate LLC for $\mathrm{SO}\left(V_{2 n}\right) \rtimes\langle\theta\rangle$ into LLC for $\mathrm{O}\left(V_{2 n}\right)$. Note that the changing of the choice of the isomorphism corresponds to the automorphism

$$
\mathrm{O}\left(V_{2 n}\right) \rightarrow \mathrm{O}\left(V_{2 n}\right), g \mapsto \operatorname{det}(g) \cdot g= \begin{cases}g & \text { if } g \in \mathrm{SO}\left(V_{2 n}\right), \\ -g & \text { otherwise. }\end{cases}
$$

Hence it induces the bijection

$$
\operatorname{Irr}\left(\mathrm{O}\left(V_{2 n}\right)\right) \rightarrow \operatorname{Irr}\left(\mathrm{O}\left(V_{2 n}\right)\right), \sigma \mapsto\left(\omega_{\sigma} \circ \text { det }\right) \otimes \sigma,
$$

where $\omega_{\sigma}$ is the central character of $\sigma$, which is regarded as a character of $\{ \pm 1\}$.

For $\delta \in\{ \pm 1\}$, we define an equivalence relation $\sim_{\text {det }}$ on $\operatorname{Irr}\left(\mathrm{O}\left(V_{2 n}^{\delta}\right)\right)$ by

$$
\sigma \sim_{\operatorname{det}} \sigma \otimes \operatorname{det}
$$

for $\sigma \in \operatorname{Irr}\left(\mathrm{O}\left(V_{2 n}^{\delta}\right)\right)$. The restriction and the induction give a canonical bijection

$$
\operatorname{Irr}\left(\mathrm{O}\left(V_{2 n}^{\delta}\right)\right) / \sim_{\operatorname{det}} \longleftrightarrow \operatorname{Irr}\left(\mathrm{SO}\left(V_{2 n}^{\delta}\right)\right) / \sim_{\varepsilon} .
$$

Put $\Phi\left(\mathrm{O}\left(V_{2 n}\right)\right)=\Phi\left(\mathrm{SO}\left(V_{2 n}\right)\right) / \sim_{\epsilon}$ and $\Phi_{*}\left(\mathrm{O}\left(V_{2 n}\right)\right)=\Phi_{*}\left(\mathrm{SO}\left(V_{2 n}\right)\right) / \sim_{\epsilon}$ for $* \epsilon$ \{temp, disc, gen $\}$. Also, we set $\Phi^{\epsilon}\left(\mathrm{O}\left(V_{2 n}\right)\right)=\Phi^{\epsilon}\left(\mathrm{SO}\left(V_{2 n}\right)\right) / \sim_{\epsilon}$. Namely, $\Phi\left(\mathrm{O}\left(V_{2 n}\right)\right)$ is the set of equivalence classes of orthogonal representations of $W D_{F}$ with dimension $2 n$ and determinant $\chi_{V}$. We call an element in $\Phi\left(\mathrm{O}\left(V_{2 n}\right)\right)$ an $L$-parameter for $\mathrm{O}\left(V_{2 n}\right)$.

We describe the local Langlands correspondence for $\mathrm{O}\left(V_{2 n}\right)$.

Desideratum 3.9 (LLC for $\mathrm{O}\left(V_{2 n}\right)$ ). Let $V_{2 n}$ be the orthogonal space associated to $(d, c)$, and $\chi_{V}=(\cdot, d)$ be the discriminant character of $V_{2 n}$.

(1) There exists a canonical surjection

$$
\bigsqcup_{\delta \in\{ \pm 1\}} \operatorname{Irr}\left(\mathrm{O}\left(V_{2 n}^{\delta}\right)\right) \rightarrow \Phi\left(\mathrm{O}\left(V_{2 n}\right)\right)
$$

For $\phi \in \Phi\left(\mathrm{O}\left(V_{2 n}\right)\right)$, we denote by $\Pi_{\phi}$ the inverse image of $\phi$ under this map, and call $\Pi_{\phi}$ the L-packet of $\phi$. 
(2) We have

$$
\bigsqcup_{\delta \in\{ \pm 1\}} \operatorname{Irr}_{*}\left(\mathrm{O}\left(V_{2 n}^{\delta}\right)\right)=\bigsqcup_{\phi \in \Phi_{*}\left(\mathrm{O}\left(V_{2 n}\right)\right)} \Pi_{\phi}
$$

for $* \in\{$ temp, disc $\}$.

(3) The following are equivalent:

- $\phi \in \Phi^{\epsilon}\left(\mathrm{O}\left(V_{2 n}\right)\right)$;

- some $\sigma \in \Pi_{\phi}$ satisfies $\sigma \otimes \operatorname{det} \neq \sigma$;

- all $\sigma \in \Pi_{\phi}$ satisfy $\sigma \otimes \operatorname{det} \neq \sigma$.

(4) For each $c^{\prime} \in F^{\times}$, there exists a bijection (not depending on $\psi$ )

$$
\iota_{c^{\prime}}: \Pi_{\phi} \rightarrow \widehat{A_{\phi}},
$$

which satisfies the (twisted) endoscopic character identities.

(5) For $\sigma \in \Pi_{\phi}$ and $c^{\prime} \in F^{\times}$, the following are equivalent:

- $\sigma \in \operatorname{Irr}\left(\mathrm{O}\left(V_{2 n}^{+}\right)\right)$;

- $\iota_{c^{\prime}}(\sigma)\left(z_{\phi}\right)=\chi_{V}\left(c^{\prime} / c\right)$.

(6) Assume that $\phi=\phi_{\tau}+\phi_{0}+\phi_{\tau}^{\vee}$, where $\phi_{0}$ is an element in $\Phi_{\text {temp }}\left(\mathrm{O}\left(V_{2 n_{0}}\right)\right)$ and $\phi_{\tau}$ is an irreducible tempered representation of $W D_{F}$ which corresponds to $\tau \in \operatorname{Irr}_{\text {temp }}\left(\operatorname{GL}_{k}(F)\right)$ with $n=n_{0}+k$. Then the induced representation

$$
\operatorname{Ind}_{P}^{\mathrm{O}\left(V_{2 n}^{\delta}\right)}\left(\tau \otimes \sigma_{0}\right)
$$

is a multiplicity-free direct sum of tempered representations of $\mathrm{O}\left(V_{2 n}^{\delta}\right)$, where $P$ is a parabolic subgroup of $\mathrm{O}\left(V_{2 n}^{\delta}\right)$ with Levi subgroup $M_{P}=\mathrm{GL}_{k}(F)$ $\times \mathrm{O}\left(V_{2 n_{0}}^{\delta}\right)$ and $\sigma_{0} \in \Pi_{\phi_{0}}$. The L-packet $\Pi_{\phi}$ is given by

$$
\Pi_{\phi}=\left\{\sigma \mid \sigma \subset \operatorname{Ind}_{P}^{\mathrm{O}\left(V_{2 n}^{\delta}\right)}\left(\tau \otimes \sigma_{0}\right) \text { for some } \sigma_{0} \in \Pi_{\phi_{0}}\right\} \text {. }
$$

Moreover if $\sigma \subset \operatorname{Ind}_{P}^{\mathrm{O}\left(V_{2 n} \delta\right)}\left(\tau \otimes \sigma_{0}\right)$, then $\iota_{c^{\prime}}(\sigma) \mid A_{\phi_{0}}=\iota_{c^{\prime}}\left(\sigma_{0}\right)$ for $c^{\prime} \in F^{\times}$.

(7) Assume that

$$
\phi=\phi_{\tau_{1}}|\cdot|{ }_{F}^{s_{1}}+\cdots+\phi_{\tau_{r}}|\cdot|{ }_{F}^{s_{r}}+\phi_{0}+\left(\phi_{\tau_{1}}|\cdot|{ }_{F}^{s_{1}}+\cdots+\phi_{\tau_{r}}|\cdot|{ }_{F}^{s_{r}}\right)^{\vee},
$$

where $\phi_{0}$ is an element in $\Phi_{\text {temp }}\left(\mathrm{O}\left(V_{2 n_{0}}^{\delta}\right)\right), \phi_{\tau_{i}}$ is an irreducible tempered representation of $W D_{F}$ which corresponds to $\tau_{i} \in \operatorname{Irr}_{\text {temp }}\left(\operatorname{GL}_{k_{i}}(F)\right)$ with $n=n_{0}+k_{1}+\cdots+k_{r}$ and $s_{i}$ is a real number with $s_{1} \geq \cdots \geq s_{r}>0$. Then the L-packet $\Pi_{\phi}$ consists of the unique irreducible quotients $\sigma$ of the standard modules

$$
\operatorname{Ind}_{P}^{\mathrm{O}\left(V_{2 n}^{\delta}\right)}\left(\tau_{1}|\operatorname{det}|_{F}^{s_{1}} \otimes \cdots \otimes \tau_{r}|\operatorname{det}|_{F}^{s_{r}} \otimes \sigma_{0}\right),
$$

where $\sigma_{0}$ runs over elements of $\Pi_{\phi_{0}}$ and $P$ is a parabolic subgroup of $\mathrm{O}\left(V_{2 n}^{\delta}\right)$ with Levi subgroup $M_{P}=\mathrm{GL}_{k_{1}}(F) \times \cdots \times \mathrm{GL}_{k_{r}}(F) \times \mathrm{O}\left(V_{2 n_{0}}^{\delta}\right)$. Moreover if $\sigma$ is the unique irreducible quotient of $\operatorname{Ind}_{P}^{\mathrm{O}\left(V_{2 n}^{\delta}\right)}\left(\tau_{1}|\operatorname{det}|{ }_{F}^{s_{1}} \otimes \cdots \otimes \tau_{r}|\operatorname{det}|_{F}^{s_{r}} \otimes\right.$ $\left.\sigma_{0}\right)$, then $\iota_{c^{\prime}}(\sigma) \mid A_{\phi_{0}}=\iota_{c^{\prime}}\left(\sigma_{0}\right)$ for $c^{\prime} \in F^{\times}$.

(8) For $\phi \in \Phi\left(\mathrm{O}\left(V_{2 n}\right)\right)=\Phi\left(\mathrm{SO}\left(V_{2 n}\right)\right) / \sim_{\epsilon}$, the image of $\Pi_{\phi}$ under the map

$$
\operatorname{Irr}\left(\mathrm{O}\left(V_{2 n}^{\delta}\right)\right) \rightarrow \operatorname{Irr}\left(\mathrm{O}\left(V_{2 n}^{\delta}\right)\right) / \sim_{\operatorname{det}} \rightarrow \operatorname{Irr}\left(\mathrm{SO}\left(V_{2 n}^{\delta}\right)\right) / \sim_{\epsilon}
$$


is the packet $\Pi_{\phi}^{0}$ in Weak $L L C$ for $\mathrm{SO}\left(V_{2 n}\right)$, and the diagram

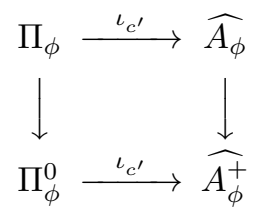

is commutative for $c^{\prime} \in F^{\times}$.

(9) For $c^{\prime} \in F^{\times}$and $\sigma \in \Pi_{\phi}$, the determinant twist $\sigma \otimes \operatorname{det}$ also belongs to $\Pi_{\phi}$, and

$$
\iota_{c^{\prime}}(\sigma \otimes \operatorname{det})(a)=\iota_{c^{\prime}}(a) \cdot(-1)^{\operatorname{det}(a)}
$$

for $a \in A_{\phi}$.

As Weak LLC for $\mathrm{SO}\left(V_{2 n}\right)$, Arthur proved the quasi-split case.

Theorem 3.10 ([ब, $\mathrm{Ar}]$. Let $V_{2 n}^{+}=V_{2 n}$ be the orthogonal space associated to $(d, c)$. Put $E=F(\sqrt{d})$. Then there exist a surjective map

$$
\operatorname{Irr}\left(\mathrm{O}\left(V_{2 n}^{+}\right)\right) \rightarrow \Phi\left(\mathrm{O}\left(V_{2 n}\right)\right)
$$

with the inverse image $\Pi_{\phi}$ of $\phi \in \Phi_{\text {temp }}\left(\mathrm{O}\left(V_{2 n}\right)\right)$, and a bijection

$$
\iota_{c^{\prime}}: \Pi_{\phi} \rightarrow\left(A_{\phi} /\left\langle z_{\phi}\right\rangle\right)^{\wedge}
$$

for $c^{\prime} \in c N_{E / F}\left(E^{\times}\right)$satisfying Desideratum 3.9 .

In fact, Arthur established Theorem 3.10 first and by using Desideratum 3.9 (8), he then defined the $L$-packets $\Pi_{\phi}^{0}$ for $\mathrm{SO}\left(V_{2 n}\right)$ (Theorem $\underline{3.6}$ ).

Remark 3.11. As we mentioned in Remark 3.3. Mœeglin [M2, §1.4, Theorem 1.4.1] and Mœglin-Renard MR2 have partially extended Theorem 3.10 to the pure inner forms as well, though it is not clear to us if all the statements in Desideratum 3.9 were verified in their work. Also, when $d \notin F^{\times 2}$, we might define $L$-packets $\Pi_{\phi}$ and a bijection $\iota_{c^{\prime}}: \Pi_{\phi} \rightarrow \widehat{A_{\phi}}$ for any $c^{\prime} \in F^{\times}$similar to Remark 3.7. However, motivated by the Prasad conjecture (Conjecture 4.2 below), we should define $\iota_{c^{\prime}}(\sigma)$ for $\sigma \in \operatorname{Irr}\left(\mathrm{O}\left(V_{2 n}^{\delta}\right)\right)$ by

$$
\iota_{c^{\prime}}(\sigma)=\iota_{c}(\sigma) \otimes \eta_{\phi \chi_{V}, c^{\prime} / c} .
$$

See also Desideratum 3.12 and Hypothesis 3.13 below.

The following is an analogue of Proposition 3.8.

Desideratum 3.12. Let $V_{2 n}$ be the orthogonal space associated to $(d, c)$. Let $\phi \in$ $\Phi\left(\mathrm{O}\left(V_{2 n}\right)\right)$ and $\sigma \in \Pi_{\phi}$. We write $\phi \chi_{V}=\phi \otimes \chi_{V}$.

(1) For $c_{1}, c_{2} \in F^{\times}$, we have

$$
\iota_{c_{2}}(\sigma)=\iota_{c_{1}}(\sigma) \otimes \eta_{\phi \chi_{V}, c_{2} / c_{1}}
$$

as a character of $A_{\phi}$.

(2) $\phi$ is generic, i.e., $L(s, \phi, \mathrm{Ad})$ is regular at $s=1$ if and only if $\Pi_{\phi}$ contains a $\mu_{c^{\prime}}^{\varepsilon}$-generic representation $\sigma$ for each $c^{\prime} \in F^{\times}$and $\varepsilon \in\{ \pm 1\}$.

(3) If $\phi$ is generic, then for each $c^{\prime} \in F^{\times}$,

- $\sigma^{+} \in \Pi_{\phi}$ is $\mu_{c^{\prime}}^{+}$-generic if and only if $\iota_{c^{\prime}}\left(\sigma^{+}\right)$is the trivial representation of $A_{\phi}$;

- $\sigma^{-} \in \Pi_{\phi}$ is $\mu_{c^{\prime}}^{-}$-generic if and only if $\iota_{c^{\prime}}\left(\sigma^{-}\right)$is given by $A_{\phi} \ni a \mapsto$ $(-1)^{\operatorname{det}(a)}$. 
(4) If both $\mathrm{O}\left(V_{2 n}^{+}\right)$and $\phi$ are unramified, then $\Pi_{\phi}$ contains a unique unramified representation $\sigma$, and it corresponds to the trivial representation of $A_{\phi}$ under $\iota_{c}$.

Under Desideratum 3.9. Proposition 3.8 and Hypothesis 3.13. Desideratum 3.12 will be proven in 33.8 below. Note that $\eta_{\phi \chi_{V}, c_{2} / c_{1}} \mid A_{\phi}^{+}=\eta_{\phi, c_{2} / c_{1}}$ since $\operatorname{dim}\left(\phi^{a}\right)$ is even for $a \in A_{\phi}^{+}$.

3.7. Intertwining relations. To establish Desideratum 3.12 and two main local theorems, we will use a very delicate hypothesis, which is an intertwining relation.

Let $V_{2 n}=V_{2 n}^{+}$be the orthogonal space associated to $(d, c)$, and $V \in\left\{V_{2 n}^{+}, V_{2 n}^{-}\right\}$. For a fixed positive integer $k$, we set

$$
X=F v_{1} \oplus \cdots \oplus F v_{k}, \quad X^{*}=F v_{1}^{*} \oplus \cdots \oplus F v_{k}^{*},
$$

to be $k$-dimensional vector spaces over $F$. Let $V^{\prime}=V \oplus X \oplus X^{*}$ be the orthogonal space defined by

$$
\left\langle v_{i}, v_{j}\right\rangle_{V^{\prime}}=\left\langle v_{i}^{*}, v_{j}^{*}\right\rangle_{V^{\prime}}=\left\langle v_{i}, v_{0}\right\rangle_{V^{\prime}}=\left\langle v_{i}^{*}, v_{0}\right\rangle_{V^{\prime}}=0, \quad\left\langle v_{i}, v_{j}^{*}\right\rangle_{V^{\prime}}=\delta_{i, j},
$$

for any $i, j=1, \ldots, k$ and $v_{0} \in V$. Let $P=M_{P} U_{P}$ be the maximal parabolic subgroup of $\mathrm{O}\left(V^{\prime}\right)$ stabilizing $X$, where $M_{P}$ is the Levi component of $P$ stabilizing $X^{*}$. Hence

$$
M_{P} \cong \mathrm{GL}(X) \times \mathrm{O}(V) .
$$

Using the basis $\left\{v_{1}, \ldots, v_{k}\right\}$ of $X$, we obtain an isomorphism $m_{P}: \mathrm{GL}_{k}(F) \rightarrow \operatorname{GL}(X)$. Let $\phi_{\tau}$ be an orthogonal tempered representation of $W D_{F}$ of dimension $k$, and $\tau$ be the tempered representation of $\mathrm{GL}_{k}(F)$ on a space $\mathcal{V}_{\tau}$ associated to $\phi_{\tau}$. For $s \in \mathbb{C}$, we realize the representation $\tau_{s}:=\tau \otimes|\operatorname{det}|_{F}^{s}$ on $\mathcal{V}_{\tau}$ by setting $\tau_{s}(a):=|\operatorname{det}(a)|_{F}^{s} \tau(a) v$ for $v \in \mathcal{V}_{\tau}$ and $a \in \mathrm{GL}_{k}(F)$. Let $\sigma$ be an irreducible tempered representation of $\mathrm{O}(V)$ on a space $\mathcal{V}_{\sigma}$. Assume that $\sigma \in \Pi_{\phi_{\sigma}}$ with $\phi_{\sigma} \in \Phi_{\text {temp }}^{\epsilon}\left(\mathrm{O}\left(V_{2 n}\right)\right)$, i.e., $\sigma \neq \sigma \otimes$ det and $\sigma \mid \mathrm{SO}(V)$ is irreducible. We define a normalized intertwining operator

$$
R_{c^{\prime}}\left(w, \tau_{s} \otimes \sigma\right): \operatorname{Ind}_{P}^{\mathrm{O}\left(V^{\prime}\right)}\left(\tau_{s} \otimes \sigma\right) \rightarrow \operatorname{Ind}_{P}^{\mathrm{O}\left(V^{\prime}\right)}\left(\tau_{s} \otimes \sigma\right)
$$

by (the meromorphic continuation of) the integral

$$
\begin{aligned}
& R_{c^{\prime}}\left(w, \tau_{s} \otimes \sigma\right) f_{s}\left(h^{\prime}\right) \\
& \quad=e(V)^{k} \cdot \chi_{V}\left(c^{\prime} / c\right)^{k} \cdot\left|c^{\prime}\right|_{F}^{k \rho_{P}} \cdot r\left(\tau_{s} \otimes \sigma\right)^{-1} \cdot \mathcal{A}_{w}\left(\int_{U_{P}} f_{s}\left(\widetilde{w}_{c^{\prime}}^{-1} u_{P} h^{\prime}\right) d u_{P}\right)
\end{aligned}
$$

for $f_{s} \in \operatorname{Ind}_{P}^{\mathrm{O}\left(V^{\prime}\right)}\left(\tau_{s} \otimes \sigma\right)$. Here,

- $w$ is the non-trivial element in the relative Weyl group $W\left(M_{P}\right)(\cong \mathbb{Z} / 2 \mathbb{Z})$ for $M_{P}$;

- $\widetilde{w}_{c^{\prime}} \in \mathrm{O}(V)$ is the representative of $w$ given by

$$
\widetilde{w}_{c^{\prime}}=w_{P} \cdot m_{P}\left(c^{\prime} \cdot a\right) \cdot\left((-1)^{k} \mathbf{1}_{V}\right),
$$

where $w_{P} \in \mathrm{O}\left(V^{\prime}\right)$ is defined by $w_{P} v_{i}=-v_{i}^{*}, w_{P} v_{i}^{*}=-v_{i}$ and $w_{P} \mid V=\mathbf{1}_{V}$, and $a \in \mathrm{GL}_{k}(F)$ is given by

$$
a=\left(\begin{array}{ccc} 
& & (-1)^{n-k+1} \\
& . &
\end{array}\right) ;
$$


- $e(V)=\iota_{c}\left(z_{\phi_{\sigma}}\right) \in\{ \pm 1\}$, i.e.,

$$
e(V)= \begin{cases}1 & \text { if } V \text { is associated to }(d, c), \\ -1 & \text { otherwise; }\end{cases}
$$

- $\rho_{P}=m+(k-1) / 2$, so that the modulus character $\delta_{P}$ of $P$ satisfies that $\delta_{P}\left(m_{P}(a)\right)=|\operatorname{det}(a)|_{F}^{2 \rho_{P}}$ for $a \in \mathrm{GL}_{k}(F)$;

- $r\left(\tau_{s} \otimes \sigma\right)$ is the normalizing factor given by

$r\left(\tau_{s} \otimes \sigma\right)$

$$
=\lambda(E / F, \psi)^{k} \frac{L\left(s, \phi_{\tau} \otimes \phi_{\sigma}\right)}{\varepsilon\left(s, \phi_{\tau} \otimes \phi_{\sigma}, \psi\right) L\left(1+s, \phi_{\tau} \otimes \phi_{\sigma}\right)} \frac{L\left(2 s, \wedge^{2} \circ \phi_{\tau}\right)}{\varepsilon\left(2 s, \wedge^{2} \circ \phi_{\tau}\right) L\left(1+2 s, \wedge^{2} \circ \phi_{\tau}\right)},
$$

where $\lambda(E / F, \psi)$ is the Langlands $\lambda$-factor associated to $E=F(\sqrt{\operatorname{disc}(V)})$ $=F(\sqrt{d})$;

- $d u_{P}$ is the Haar measure of $U_{P}$ given in [Ar] (see also [At1, §6]);

- $\mathcal{A}_{w}=\mathcal{A}_{\tau, w} \otimes \mathbf{1}_{\mathcal{V}_{\sigma}}: \mathcal{V}_{\tau} \otimes \mathcal{V}_{\sigma} \rightarrow \mathcal{V}_{\tau} \otimes \mathcal{V}_{\sigma}$ is an intertwining isomorphism such that

$$
\mathcal{A}_{w} \circ(\tau \otimes \sigma)\left(\widetilde{w}_{c^{\prime}}^{-1} m \widetilde{w}_{c^{\prime}}\right)=(\tau \otimes \sigma)(m) \circ \mathcal{A}_{w}
$$

for $m \in M_{P}$, and $\Lambda \circ \mathcal{A}_{\tau, w}=\Lambda$ for a fixed non-zero Whittaker functional $\Lambda$ on $\mathcal{V}_{\tau}$ with respect to the Whittaker datum $\left(B_{k}, \psi_{U_{k}}\right)$, where $B_{k}=T_{k} U_{k}$ is the Borel subgroup consisting of upper triangular matrices in $\mathrm{GL}_{k}(F)$ and $\psi_{U_{k}}$ is the generic character of the unipotent radical $U_{k}$ of $B_{k}$ given by $\psi_{U_{k}}=\psi\left(x_{1,2}+\cdots+x_{k-1, k}\right)$.

We expect that the intertwining operators and the local Langlands correspondence are related as follows:

Hypothesis 3.13. Notation is as above.

(1) The normalized intertwining operator $R_{c^{\prime}}\left(w, \tau_{s} \otimes \sigma\right)$ is holomorphic at $s=0$. We put $R_{c^{\prime}}(w, \tau \otimes \sigma):=R_{c^{\prime}}\left(w, \tau_{0} \otimes \sigma\right)$.

(2) Suppose that $\phi_{\tau}$ is a multiplicity-free sum of irreducible orthogonal tempered representations. Put $\phi_{\sigma^{\prime}}=\phi_{\tau} \oplus \phi_{\sigma} \oplus \phi_{\tau}$, and denote by $a \in A_{\phi_{\sigma^{\prime}}}$ the element corresponding to $\phi_{\tau}$. Let $\sigma^{\prime} \in \Pi_{\phi_{\sigma^{\prime}}}$ be an irreducible constituent of $\operatorname{Ind}_{P}^{\mathrm{O}\left(V^{\prime}\right)}(\tau \otimes \sigma)$. Then we have

$$
R_{c^{\prime}}(w, \tau \otimes \sigma) \mid \sigma^{\prime}=\iota_{c^{\prime}}\left(\sigma^{\prime}\right)(a)
$$

for any $c^{\prime} \in F^{\times}$.

In special cases, Hypothesis 3.13 has been established:

Theorem 3.14. Hypothesis 3.13 holds in the following cases:

- The case when $V=V_{2 n}^{+}$and $c^{\prime} \in c N_{E / F}\left(E^{\times}\right)$.

- The case when $k$ is even and $d \neq 1$ in $F^{\times} / F^{\times 2}$ provided Desideratum 3.5 holds.

Proof. In the first case, Hypothesis 3.13 is Proposition 2.3.1 and Theorems 2.2.1, 2.2.4, 2.4.1 and 2.4.4 in $\mathrm{Ar}$. For the detail, see e.g., At2, Theorem 2.2]. The second case follows from Arthur's results above and [At1, Proposition 3.4].

The cases when Hypothesis 3.13 has not yet been verified are

- the non-quasi-split even orthogonal case; and

- the case when $k$ is odd and $d \neq 1$ in $F^{\times} / F^{\times 2}$. 
In general, Hypothesis 3.13 would follow from similar results to $\mathrm{Ar}$ and [KMSW].

Remark 3.15. Recall that we need to choose an isomorphism

$$
\mathrm{O}(V) \longleftrightarrow \mathrm{SO}(V) \rtimes\langle\theta\rangle
$$

to translate Arthur's result. There exist two choices of isomorphisms, which are determined by $\pm \epsilon \leftrightarrow \theta$. We have chosen the isomorphism such that $\epsilon \leftrightarrow \theta$. If one chooses the other isomorphism $-\epsilon \leftrightarrow \theta$, one should replace the representative $\widetilde{w}_{c^{\prime}}$ of $w \in W\left(M_{P}\right)$ as

$$
\widetilde{w}_{c^{\prime}}^{\prime}=-w_{P} \cdot m_{P}\left(c^{\prime} \cdot a\right) \cdot\left((-1)^{k} \mathbf{1}_{V}\right)=-\widetilde{w}_{c^{\prime}} .
$$

Note that

$$
f_{s}\left(\widetilde{w}_{c^{\prime}}^{\prime} u_{P} h^{\prime}\right)=\omega_{s}(-1) \cdot f_{s}\left(\widetilde{w}_{c^{\prime}} u_{P} h^{\prime}\right)
$$

for $f_{s} \in \operatorname{Ind}_{P}^{\mathrm{O}\left(V^{\prime}\right)}\left(\tau_{s} \otimes \sigma\right)$, where $\omega_{s}$ is the central character of $\operatorname{Ind}_{P}^{\mathrm{O}\left(V^{\prime}\right)}\left(\tau_{s} \otimes \sigma\right)$. This is compatible with the bijection

$$
\operatorname{Irr}(\mathrm{O}(V)) \rightarrow \operatorname{Irr}(\mathrm{O}(V)), \sigma \mapsto\left(\omega_{\sigma} \circ \operatorname{det}\right) \otimes \sigma,
$$

as in 3.6. Hence all results below are independent of the choice of the isomorphism $\mathrm{O}(V) \cong \mathrm{SO}(V) \rtimes\langle\theta\rangle$.

An analogous property to Hypothesis 3.13 holds for symplectic groups. We take the symplectic space $W_{2 n}$ of dimension $2 n$. Let $\phi_{\tau}$ be an orthogonal tempered representation of $W D_{F}$, and $\tau \in \operatorname{Irr}\left(\mathrm{GL}_{k}(F)\right)$ be the tempered representation corresponding to $\phi_{\tau}$. In $\mathrm{Ar}$, Arthur has defined a normalized intertwining operator $R\left(w^{\prime}, \tau \otimes \pi\right)$ on $\operatorname{Ind}_{Q}^{\operatorname{Sp}\left(W_{2 n^{\prime}}\right)}(\tau \otimes \pi)$ for $\pi \in \operatorname{Irr}_{\text {temp }}\left(\operatorname{Sp}\left(W_{2 n}\right)\right)$, where $n^{\prime}=n+k$ and $Q$ is a parabolic subgroup of $\operatorname{Sp}\left(W_{2 n^{\prime}}\right)$ whose Levi subgroup is $M_{Q} \cong \operatorname{GL}_{k}(F) \times \operatorname{Sp}\left(W_{2 n}\right)$. See also [At1, $\left.\S 6\right]$. Note that $\operatorname{Ind}_{Q}^{\operatorname{Sp}\left(W_{2 n^{\prime}}\right)}(\tau \otimes \pi)$ is multiplicity-free.

Recall that if $\phi^{\prime} \in \Phi_{\text {temp }}\left(\operatorname{Sp}\left(W_{2 n^{\prime}}\right)\right)$ satisfies that $\phi^{\prime}=\phi_{\tau} \oplus \phi \oplus \phi_{\tau}$ for some $\phi \in \Phi_{\text {temp }}\left(\operatorname{Sp}\left(W_{2 n}\right)\right)$, then the $L$-packet $\Pi_{\phi^{\prime}}$ is given by

$$
\Pi_{\phi^{\prime}}=\left\{\pi^{\prime} \mid \pi^{\prime} \subset \operatorname{Ind}_{Q}^{\mathrm{Sp}\left(W_{2 n^{\prime}}\right)}(\tau \otimes \pi) \text { for some } \pi \in \Pi_{\phi}\right\} .
$$

Also we recall that there exists a bijection

$$
\iota_{c^{\prime}}: \Pi_{\phi^{\prime}} \rightarrow\left(A_{\phi} /\left\langle z_{\phi}\right\rangle\right)^{\wedge}
$$

for $c^{\prime} \in F^{\times}$. See Theorem 3.2 and Remark 3.3. An analogue of Hypothesis 3.13 is given as follows:

Proposition 3.16. Let $\phi_{\pi} \in \Phi_{\text {temp }}\left(\operatorname{Sp}\left(W_{2 n}\right)\right)$ and $\pi \in \Pi_{\phi_{\pi}}$. We put $\phi_{\pi^{\prime}}=\phi_{\tau} \oplus$ $\phi_{\pi} \oplus \phi_{\tau}$. We denote by $a^{\prime} \in A_{\phi_{\pi^{\prime}}}$ the element corresponding to $\phi_{\tau}$. Let $\pi^{\prime}$ be an irreducible constituent of $\operatorname{Ind}_{Q}^{\operatorname{Sp}\left(W_{2 n^{\prime}}\right)}(\tau \otimes \pi)$. Then we have

$$
R\left(w^{\prime}, \tau \otimes \pi\right) \mid \pi^{\prime}=\iota_{1}\left(\pi^{\prime}\right)\left(a^{\prime}\right) .
$$

Proof. This follows from Theorems 2.2.1 and 2.4.1 in [Ar]. For the detail, see e.g., At2, Theorem 2.2]. 
3.8. Proof of Desideratum 3.12. In this subsection, we prove Desideratum 3.12 under Hypothesis 3.13. First, we treat the tempered case.

Theorem 3.17. Assume Desiderata 3.5, 3.9 and Hypothesis 3.13. Then Desideratum 3.12 holds for $\phi \in \Phi_{\text {temp }}(\mathrm{O}(V))$. In particular, it holds for the L-packets $\Pi_{\phi} \cap \operatorname{Irr}\left(\mathrm{O}\left(V_{2 n}^{+}\right)\right)$of quasi-split $\mathrm{O}\left(V_{2 n}^{+}\right)$and $c_{1}, c_{2}, c^{\prime} \in c N_{E / F}\left(E^{\times}\right)$unconditionally.

Proof. Note that Proposition 3.8 holds since we assume Desideratum 3.5

First, we consider (1). Let $\phi \in \Phi_{\text {temp }}(\mathrm{O}(V))$ and $\sigma \in \Pi_{\phi}$. We have to show that

$$
\iota_{c_{1}}(\sigma)(a)=\iota_{c_{2}}(\sigma)(a) \cdot \operatorname{det}\left(\phi^{a} \chi_{V}\right)\left(c_{1} / c_{2}\right)
$$

for any $a \in A_{\phi}$ and $c_{1}, c_{2} \in F^{\times}$. Fix $a \in A_{\phi}$ and consider the parameter

$$
\phi^{\prime}=\phi^{a} \oplus \phi \oplus \phi^{a} .
$$

Let $\tau \in \operatorname{Irr}\left(\mathrm{GL}_{k}(F)\right)$ be the representation corresponding to $\phi^{a}$, where $k=\operatorname{dim}\left(\phi^{a}\right)$, and put $\sigma^{\prime}=\operatorname{Ind}_{P}^{\mathrm{O}\left(V^{\prime}\right)}(\tau \otimes \sigma)$ as above. Then $A_{\phi}=A_{\phi^{\prime}}$ since $\phi$ contains $\phi^{a}$. Hence $\sigma^{\prime}$ is irreducible and $\sigma^{\prime} \in \Pi_{\phi^{\prime}}$ by Desideratum 3.9 (6). Moreover, we have

$$
\iota_{c^{\prime}}\left(\sigma^{\prime}\right) \mid A_{\phi}=\iota_{c^{\prime}}(\sigma)
$$

for any $c^{\prime} \in F^{\times}$. By Hypothesis 3.13, $R_{c_{i}}(w, \tau \otimes \sigma)$ is the scalar operator with eigenvalue $\iota_{c_{i}}(\sigma)(a)$ for $i=1,2$. By definition, we have

$$
R_{c_{1}}(w, \tau \otimes \sigma)=\chi_{V}\left(c_{1} / c_{2}\right)^{k} \cdot \omega_{\tau}\left(c_{1} / c_{2}\right) \cdot R_{c_{2}}(w, \tau \otimes \sigma),
$$

where $\omega_{\tau}$ is the central character of $\tau$, which is equal to $\operatorname{det}\left(\phi^{a}\right)$. Since

$$
\chi_{V}\left(c_{1} / c_{2}\right)^{k} \cdot \omega_{\tau}\left(c_{1} / c_{2}\right)=\operatorname{det}\left(\phi^{a} \chi_{V}\right)\left(c_{1} / c_{2}\right),
$$

we have

as desired.

$$
\iota_{c_{1}}(\sigma)(a)=\operatorname{det}\left(\phi^{a} \chi_{V}\right)\left(c_{1} / c_{2}\right) \cdot \iota_{c_{2}}(\sigma)(a)
$$

The assertion (2) follows from Lemma 2.3 and Proposition 3.8 (2).

Next, we consider (3). Let $\phi \in \Phi_{\text {temp }}(\mathrm{O}(V))$. Note that $\phi$ is generic. By Proposition 3.8 (2) and Desideratum 3.12 (2), for each $c^{\prime} \in F^{\times}$, there exists a $\mu_{c^{\prime}}^{+}$-generic representation $\sigma \in \Pi_{\phi}$ such that $\iota_{c^{\prime}}(\sigma) \mid A_{\phi}^{+}=\mathbf{1}$. We may assume that $\sigma$ is a representation of $\mathrm{O}(V)$ with $V$ associated to $\left(d, c^{\prime}\right)$. We have to show that $\iota_{c^{\prime}}(\sigma)=1$. If $\phi \notin \Phi^{\epsilon}(\mathrm{O}(V))$, then $A_{\phi}^{+}=A_{\phi}$ so that we have nothing to prove. Hence we may assume that $\phi$ contains an irreducible orthogonal representation $\phi_{0}$ with odd dimension $k$. Let $a_{0} \in A_{\phi}$ be the element corresponding to $\phi_{0}$. Now we consider the parameter

$$
\phi^{\prime}=\phi_{0} \oplus \phi \oplus \phi_{0} .
$$

For $s \in \mathbb{C}$, let $\tau_{s}=\tau|\cdot|_{F}^{s} \in \operatorname{Irr}\left(\mathrm{GL}_{k}(F)\right)$ be the representation corresponding to $\phi_{0}|\cdot|_{F}^{s}$. We may assume that $\tau_{s}$ is realized on a space $\mathcal{V}_{\tau}$, which is independent of $s \in \mathbb{C}$. Put $\sigma_{s}^{\prime}=\operatorname{Ind}_{P}^{\mathrm{O}\left(V^{\prime}\right)}\left(\tau_{s} \otimes \sigma\right)$ as above. Then by Desideratum 3.9 (6), $\sigma_{0}^{\prime}$ is irreducible and $\sigma_{0}^{\prime} \in \Pi_{\phi^{\prime}}$. Moreover, the canonical injection $A_{\phi} \hookrightarrow A_{\phi^{\prime}}$ is bijective, and we have

$$
\iota_{c^{\prime}}\left(\sigma_{0}^{\prime}\right) \mid A_{\phi}=\iota_{c^{\prime}}(\sigma) .
$$

Note that $\tau=\tau_{0}$ is tempered, so it is generic. Fix a non-zero homomorphism

$$
l: \tau \otimes \sigma \rightarrow \mathbb{C}
$$

such that

$$
l(\sigma(u) v)=\mu_{c^{\prime}}^{+}(u) \ell(v)
$$


for $u \in U^{\prime} \cap M_{P}$ and $v \in \tau \otimes \sigma$, where $U^{\prime}=U_{0}^{\prime} \rtimes\langle\epsilon\rangle$ with the maximal unipotent subgroup $U_{0}^{\prime}$ of $\mathrm{SO}\left(V^{\prime}\right)$ as in $₫ 2.2$, and $M_{P}=\mathrm{GL}_{k}(F) \times \mathrm{O}(V)$ is the Levi subgroup of $P$. For $f_{s} \in \sigma_{s}^{\prime}$, we put

$$
l_{s}\left(f_{s}\right)=\int_{U_{0}} l\left(f_{s}\left(\widetilde{w}_{c^{\prime}}^{-1} u_{0}\right)\right) \mu_{c^{\prime}}^{-1}\left(u_{0}\right) d u_{0},
$$

where $\widetilde{w}_{c^{\prime}} \in \mathrm{SO}(V)$ is the representative of $w$ defined in 43.7 . Then by $\mathrm{CS}$, Proposition 2.1] and [S1, Proposition 3.1], $l_{s}\left(f_{s}\right)$ is absolutely convergent for $\operatorname{Re}(s) \gg 0$, and has analytic continuation to $\mathbb{C}$. Moreover, $l_{0}$ gives a non-zero map

$$
l_{0}: \sigma_{0}^{\prime} \rightarrow \mathbb{C}
$$

such that

$$
l_{0}\left(\sigma_{0}^{\prime}\left(u^{\prime}\right) f_{0}\right)=\mu_{c^{\prime}}^{+}\left(u^{\prime}\right) l_{0}\left(f_{0}\right)
$$

for $u^{\prime} \in U^{\prime}$ and $f_{0} \in \sigma_{0}^{\prime}$. By a result of Shahidi ([S2, Theorem 3.5]), we have

$$
l_{0} \circ R_{c^{\prime}}(w, \tau \otimes \sigma)=l_{0} .
$$

See also $\mathrm{Ar}$, Theorem 2.5.1]. This equation together with Hypothesis 3.13 shows that

$$
\iota_{c^{\prime}}(\sigma)\left(a_{0}\right)=\iota_{c^{\prime}}\left(\sigma_{0}^{\prime}\right)\left(a_{0}\right)=1 .
$$

Since $\left[A_{\phi}: A_{\phi}^{+}\right]=2$, we have $\iota_{c^{\prime}}(\sigma)=\mathbf{1}$, as desired.

Finally, we consider (4). Suppose that $\mathrm{O}(V)$ and $\phi \in \Phi_{\text {temp }}(\mathrm{O}(V))$ are unramified. By Desideratum 3.5 and Lemma 2.4. $\Pi_{\phi}$ contains a unique unramified representation $\sigma$, which satisfies that $\iota_{c}(\sigma) \mid A_{\phi}^{+}=\mathbf{1}$. We have to show that $\iota_{c}(\sigma)=\mathbf{1}$. We may assume that $\phi \in \Phi^{\epsilon}(\mathrm{O}(V))$. Since $\iota_{c}(\sigma) \mid A_{\phi}^{+}=\mathbf{1}$, by Proposition 3.8 (3), we see that $\sigma$ is $\mu_{c}$-generic, i.e., there is a non-zero homomorphism $l: \sigma \rightarrow \mathbb{C}$ such that $l\left(\sigma\left(u_{0}\right) v\right)=\mu_{c}\left(u_{0}\right) l(v)$ for $u_{0} \in U_{0}$ and $v \in \sigma$. By the Casselman-Shalika formula [CS, Theorem 5.4], we have $l \mid \sigma^{K_{0}} \neq 0$, i.e., if $v \in \sigma$ is a non-zero $K_{0}$-fixed vector, then $l(v) \neq 0$. Since $\sigma \otimes \operatorname{det} \neq \sigma$, we have

$$
l \in \operatorname{Hom}_{U}\left(\sigma, \mu_{c}^{\delta}\right)
$$

for some $\delta \in\{ \pm 1\}$. However, if $v \in \sigma$ is a non-zero $K$-fixed vector, then we have

$$
\delta \cdot l(v)=\mu_{c}^{\delta}(\epsilon) \cdot l(v)=l(\sigma(\epsilon) v)=l(v) .
$$

This shows that $\sigma$ is $\mu_{c}^{+}$-generic, and so that $\iota_{c}(\sigma)=\mathbf{1}$ by Lemma 2.3 and Desideratum $3.12(3)$.

Now we treat the general case.

Corollary 3.18. Assume Desiderata 3.5, 3.9 and Hypothesis 3.13, Then Desideratum 3.12 holds in general. In particular, it holds for the L-packets $\Pi_{\phi} \cap \operatorname{Irr}\left(\mathrm{O}\left(V_{2 n}^{+}\right)\right)$ of quasi-split $\mathrm{O}\left(V_{2 n}^{+}\right)$and $c_{1}, c_{2}, c^{\prime} \in c N_{E / F}\left(E^{\times}\right)$unconditionally.

Proof. This follows from the compatibility of LLC and the Langlands quotients (Desideratum $3.9(7)$ ).

Remark 3.19. (1) Kaletha proved Proposition 3.8 (1) in [Ka1, Theorem 3.3] by comparing transfer factors. One may feel that the proof of Desideratum 3.12 (1) (Theorem 3.17) differs from Kaletha's proof. However to prove Hypothesis 3.13 , one would need a similar argument to Ka1. Hence the proof of Desideratum 3.12 (1) would be essentially the same as the one of [Ka1, Theorem 3.3]. 
(2) In At2, the first author gave a proof of the "only if" part of Proposition 3.8 (3). This proof is essentially the same as the proof of Desideratum 3.12 (3) (Theorem 3.17).

\section{Prasad's CONJECTURE}

Prasad's conjecture describes precisely the local theta correspondence for $\left(\mathrm{O}\left(V_{2 n}\right), \mathrm{Sp}\left(W_{2 n}\right)\right)$ in terms of the local Langlands correspondence for $\mathrm{O}\left(V_{2 n}\right)$ and $\operatorname{Sp}\left(W_{2 n}\right)$. A weaker version of this conjecture has been proven by the first author At1. In this section, we state Prasad's conjecture and give a proof for the full version.

4.1. Local theta correspondence. We introduce the local theta correspondence induced by the Weil representation $\omega_{W, V, \psi}$ of $\operatorname{Sp}\left(W_{2 m}\right) \times \mathrm{O}\left(V_{2 n}\right)$, and recall some basic general results.

We have fixed a non-trivial additive character $\psi$ of $F$. We denote the Weil representation of $\operatorname{Sp}\left(W_{2 m}\right) \times \mathrm{O}\left(V_{2 n}\right)$ by $\omega=\omega_{W, V, \psi}$. Let $\sigma \in \operatorname{Irr}\left(\mathrm{O}\left(V_{2 n}\right)\right)$. Then the maximal $\sigma$-isotypic quotient of $\omega$ is of the form

$$
\Theta(\sigma) \otimes \sigma,
$$

where $\Theta(\sigma)=\Theta_{W, V, \psi}(\sigma)$ is a smooth representation of $\operatorname{Sp}\left(W_{2 m}\right)$. It was shown by Kudla $\mathrm{Ku}$ that $\Theta(\sigma)$ has finite length (possibly zero). The maximal semi-simple quotient of $\Theta(\sigma)$ is denoted by $\theta(\sigma)=\theta_{W, V, \psi}(\sigma)$.

Similarly, for $\pi \in \operatorname{Irr}\left(\operatorname{Sp}\left(W_{2 m}\right)\right)$, we obtain smooth finite length representations $\Theta(\pi)=\Theta_{V, W, \psi}(\pi)$ and $\theta(\pi)=\theta_{V, W, \psi}(\pi)$ of $\mathrm{O}\left(V_{2 n}\right)$. The Howe duality conjecture, which was proven by Waldspurger [W1 if the residue characteristic is not 2 and by Gan-Takeda [GT1, GT2 in general, says that $\theta(\sigma)$ and $\theta(\pi)$ are irreducible (if they are non-zero).

4.2. Prasad's conjecture. Let $V$ be the orthogonal space associated to $(d, c)$, and $W$ be the symplectic space with $\operatorname{dim}(V)=\operatorname{dim}(W)=2 n$. We denote the discriminant character of $V$ by $\chi_{V}$. Let $\phi \in \Phi(\mathrm{O}(V))$, and put

$$
\phi^{\prime}=(\phi \oplus \mathbf{1}) \otimes \chi_{V} .
$$

Then we have $\phi^{\prime} \in \Phi(\operatorname{Sp}(W))$. Moreover we have a canonical injection $A_{\phi} \hookrightarrow A_{\phi^{\prime}}$. We denote the image of $a \in A_{\phi}$ by $a^{\prime} \in A_{\phi^{\prime}}$. One should not confuse $z_{\phi}^{\prime}$ with $z_{\phi^{\prime}}$. They satisfy $z_{\phi}^{\prime}=e_{1}^{\prime}+z_{\phi^{\prime}}$, where $e_{1}^{\prime} \in A_{\phi^{\prime}}$ is the element corresponding to $\chi_{V} \subset \phi^{\prime}$.

Lemma 4.1. For any $\phi \in \Phi(\mathrm{O}(V))$, the map

$$
A_{\phi} \hookrightarrow A_{\phi^{\prime}} \rightarrow A_{\phi^{\prime}} /\left\langle z_{\phi^{\prime}}\right\rangle
$$

is surjective. It is not injective if and only if $\phi$ contains 1 . In this case, the kernel of this map is generated by $e_{1}+z_{\phi}$, where $e_{1} \in A_{\phi}$ is the element corresponding to 1.

Proof. The map $A_{\phi} \hookrightarrow A_{\phi^{\prime}}$ is not surjective if and only if $\phi$ does not contain 1 . In this case the cokernel of this map is generated by $e_{1}^{\prime}$. Since $z_{\phi}^{\prime}=e_{1}^{\prime}+z_{\phi^{\prime}}$, we have the surjectivity of $A_{\phi} \hookrightarrow A_{\phi^{\prime}} \rightarrow A_{\phi^{\prime}} /\left\langle z_{\phi^{\prime}}\right\rangle$.

By comparing the order of $A_{\phi}$ with the one of $A_{\phi^{\prime}} /\left\langle z_{\phi^{\prime}}\right\rangle$, we see that $A_{\phi} \hookrightarrow$ $A_{\phi^{\prime}} \rightarrow A_{\phi^{\prime}} /\left\langle z_{\phi^{\prime}}\right\rangle$ is not injective if and only if $\phi$ contains 1 . In this case, the order of the kernel is 2 . Since $\left(e_{1}+z_{\phi}\right)^{\prime}=e_{1}^{\prime}+z_{\phi}^{\prime}=z_{\phi^{\prime}}$ the kernel is generated by $e_{1}+z_{\phi}$. 
Prasad's conjecture is stated as follows:

Conjecture 4.2 (Prasad's conjecture for $\left.\left(\mathrm{O}\left(V_{2 n}\right), \operatorname{Sp}\left(W_{2 n}\right)\right)\right)$. Let $V=V^{+}$and $W$ be the orthogonal space associated to $(d, c)$ and the symplectic space with $\operatorname{dim}(V)=$ $\operatorname{dim}(W)=2 n$, respectively. We denote by $\chi_{V}=\chi_{d}$ the discriminant character of $V$. Let $\phi \in \Phi(\mathrm{O}(V))$ and put $\phi^{\prime}=(\phi \oplus \mathbf{1}) \otimes \chi_{V} \in \Phi(\operatorname{Sp}(W))$. For $\sigma \in \Pi_{\phi} \cap \operatorname{Irr}\left(\mathrm{O}\left(V^{\delta}\right)\right)$ with $\delta \in\{ \pm 1\}$, we have the following:

(1) $\Theta_{W, V^{\delta}, \psi}(\sigma)$ is zero if and only if $\phi$ contains 1 and $\iota_{c^{\prime}}(\sigma)\left(z_{\phi}+e_{1}\right)=-1$, where $e_{1} \in A_{\phi}$ is the element corresponding to $\mathbf{1} \subset \phi$.

(2) Assume that $\pi=\theta_{W, V^{\delta}, \psi}(\sigma)$ is non-zero. Then $\pi \in \Pi_{\phi^{\prime}}$ and $\iota_{c^{\prime}}(\pi) \mid A_{\phi}=$ $\iota_{c^{\prime}}(\sigma)$ for $c^{\prime} \in F^{\times}$.

Remark 4.3. (1) Recall that for $\pi \in \Pi_{\phi^{\prime}} \subset \operatorname{Irr}\left(\operatorname{Sp}\left(W_{2 n}\right)\right)$, the character $\iota_{c^{\prime}}(\pi)$ of $A_{\phi^{\prime}}$ factors through $A_{\phi^{\prime}} /\left\langle z_{\phi^{\prime}}\right\rangle$. By Lemma 4.1, we see that $\iota_{c^{\prime}}(\pi)$ is determined completely by its restriction to $A_{\phi}$.

(2) By [GI1, Theorem C.5], we know that

- if $\phi$ does not contain $\mathbf{1}$, then both $\Theta_{W, V^{\delta}, \psi}(\sigma)$ and $\Theta_{W, V^{\delta}, \psi}(\sigma \otimes$ det $)$ are non-zero;

- if $\phi$ contains $\mathbf{1}$, then exactly one of $\Theta_{W, V^{\delta}, \psi}(\sigma)$ or $\Theta_{W, V^{\delta}, \psi}(\sigma \otimes$ det $)$ is non-zero;

- if $\pi=\theta_{W, V^{\delta}, \psi}(\sigma)$ is non-zero, then $\pi \in \Pi_{\phi^{\prime}}$.

Hence Conjecture 4.2 (1) follows from (2) since $z_{\phi}^{\prime}=e_{1}^{\prime}+z_{\phi^{\prime}}$.

The first main theorem is as follows:

Theorem 4.4. Assume Desideratum 3.9 and Hypothesis 3.13 , Then Prasad's conjecture for $\left(\mathrm{O}\left(V_{2 n}\right), \operatorname{Sp}\left(W_{2 n}\right)\right)$ (Conjecture 4.2) holds. In particular, it holds unconditionally when $\sigma \in \operatorname{Irr}\left(\mathrm{O}\left(V^{+}\right)\right)$and $c^{\prime} \in c N_{E / F}\left(E^{\times}\right)$with $E=F(\sqrt{d})$.

A weaker version of Prasad's conjecture (Conjecture 4.2), which is formulated by using Weak LLC for $\mathrm{SO}(V)$ or its translation into $\mathrm{O}(V)$ (i.e., by using $A_{\phi}^{+}$), was proven by [At1] under Desideratum 3.5 and Hypothesis 3.13

Theorem 4.5 ([At1, §5.5]). Assume Desideratum 3.5 and Hypothesis 3.13 for even $k$. Let $\phi \in \Phi(\mathrm{O}(V))$ and put $\phi^{\prime}=(\phi \oplus \mathbf{1}) \otimes \chi_{V} \in \Phi(\operatorname{Sp}(W))$ as in Conjecture 4.2 . For $\sigma \in \Pi_{\phi} \cap \operatorname{Irr}\left(\mathrm{O}\left(V^{\delta}\right)\right)$ with $\delta \in\{ \pm 1\}$, if $\pi=\theta_{W, V^{\delta}, \psi}(\sigma)$ is non-zero, then $\pi \in \Pi_{\phi^{\prime}}$ and

$$
\iota_{c^{\prime}}(\pi)\left|A_{\phi}^{+}=\iota_{c^{\prime}}(\sigma)\right| A_{\phi}^{+}
$$

for $c^{\prime} \in F^{\times}$. In particular, the same holds unconditionally when $\sigma \in \operatorname{Irr}\left(\mathrm{O}\left(V^{+}\right)\right)$ and $c^{\prime} \in c N_{E / F}\left(E^{\times}\right)$with $E=F(\sqrt{d})$.

We may consider the theta correspondence for $\left(\operatorname{Sp}\left(W_{2 n-2}\right), \mathrm{O}\left(V_{2 n}\right)\right)$. There is also Prasad's conjecture for $\left(\mathrm{Sp}\left(W_{2 n-2}\right), \mathrm{O}\left(V_{2 n}\right)\right)$.

Conjecture 4.6 (Prasad's conjecture for $\left.\left(\operatorname{Sp}\left(W_{2 n-2}\right), \mathrm{O}\left(V_{2 n}\right)\right)\right)$. Let $V$ be the orthogonal space associated to $(d, c)$ with $\operatorname{dim}(V)=2 n$, and $W$ be the symplectic space with $\operatorname{dim}(W)=2 n-2$. We denote by $\chi_{V}=\chi_{d}$ the discriminant character of $V$. Let $\phi^{\prime} \in \Phi(\operatorname{Sp}(W))$ and put $\phi=\left(\phi^{\prime} \otimes \chi_{V}\right) \oplus \mathbf{1} \in \Phi(\mathrm{O}(V))$. For $\delta \in\{ \pm 1\}$, we put

$$
e\left(V^{\delta}\right)=\delta \cdot \chi_{V}\left(c^{\prime} / c\right) \text {. }
$$

Let $\pi \in \Pi_{\phi^{\prime}}$.

(1) $\Theta_{V^{\delta}, W, \psi}(\pi)=0$ if and only if $\phi^{\prime}$ contains $\chi_{V}$ and $\iota_{c^{\prime}}(\pi)\left(e_{1}+z_{\phi^{\prime}}\right)=-e\left(V^{\delta}\right)$. 
(2) Assume that $\sigma=\theta_{V^{\delta}, W, \psi}(\pi)$ is non-zero. Then $\sigma \in \Pi_{\phi}$ and so there is a canonical injection $A_{\phi^{\prime}} \hookrightarrow A_{\phi}$. Moreover, $\iota_{c^{\prime}}(\sigma)$ satisfies that

- $\iota_{c^{\prime}}(\sigma)\left(z_{\phi}\right)=e\left(V^{\delta}\right)$;

- $\iota_{c^{\prime}}(\sigma) \mid A_{\phi^{\prime}}=\iota_{c^{\prime}}(\pi)$ for $c^{\prime} \in F^{\times}$.

The following theorem shows that Conjecture 4.2 implies Conjecture 4.6 .

Theorem 4.7. Assume Desideratum 3.9 and Hypothesis 3.13 (so that Conjecture 4.2 holds by Theorem 4.4). Then Prasad's conjecture for $\left(\operatorname{Sp}\left(W_{2 n-2}\right), \mathrm{O}\left(V_{2 n}\right)\right)$ (Conjecture 4.6]) holds. In particular, it holds unconditionally when $\delta=+1$ and $c^{\prime} \in c N_{E / F}\left(E^{\times}\right)$with $E=F(\sqrt{d})$.

Proof. The equation $\iota_{c^{\prime}}(\sigma)\left(z_{\phi}\right)=e\left(V^{+}\right)$follows from Desideratum 3.9 (5) and Proposition 3.8 (1). Under assuming Desideratum 3.5 and Hypothesis 3.13 for even $k$, the first author showed that $\iota_{c^{\prime}}(\sigma)\left|A_{\phi^{\prime}}^{+}=\iota_{c^{\prime}}(\pi)\right| A_{\phi^{\prime}}^{+}$for $c^{\prime} \in F^{\times}$([At1, Theorem 1.7]). Hence it suffices to show the equation

$$
\iota_{c^{\prime}}(\sigma)\left(e_{1}+z_{\phi}\right)=1
$$

where $e_{1}$ is the element of $A_{\phi}$ corresponding to $\mathbf{1}$. This equation follows from Conjecture 4.2 (Theorem 4.4) together with the tower property (see [Ku]).

4.3. Proof of Prasad's conjecture. In this subsection, we prove Theorem 4.4.

Recall that there is a sequence

$$
\Phi_{\text {temp }}^{\epsilon}(\mathrm{O}(V)) \subset \Phi_{\text {temp }}(\mathrm{O}(V)) \subset \Phi(\mathrm{O}(V)) .
$$

First, we reduce Conjecture 4.2 to the case when $\phi \in \Phi_{\text {temp }}^{\epsilon}(\mathrm{O}(V))$.

Lemma 4.8. If Prasad's conjecture (Conjecture 4.2) holds for any $\phi_{0} \in \Phi_{\text {temp }}(\mathrm{O}(V))$, then it holds for any $\phi \in \Phi(\mathrm{O}(V))$.

Proof. This follows from a compatibility of LLC, Langlands quotients and theta lifts (Desideratum 3.1 (6) and [GI1, Proposition C.4]).

Lemma 4.9. Assume Desideratum 3.5 and Hypothesis 3.13. Then Prasad's conjecture (Conjecture 4.2) holds for any $\phi \in \Phi_{\text {temp }}(\mathrm{O}(V)) \backslash \Phi_{\text {temp }}^{\epsilon}(\mathrm{O}(V))$.

Proof. Since $A_{\phi}^{+}=A_{\phi}$, this follows from Theorem 4.5

Hence Prasad's conjecture (Conjecture 4.2) is reduced to the case when $\phi \in$ $\Phi_{\text {temp }}^{\epsilon}(\mathrm{O}(V))$. For this case, the following is the key proposition:

Proposition 4.10. Let $V_{2 n}=V_{2 n}^{+}$and $W_{2 n}$ be the orthogonal space associated to $(d, c)$ and the symplectic space with $\operatorname{dim}\left(V_{2 n}\right)=\operatorname{dim}\left(W_{2 n}\right)=2 n$, respectively. Fix a positive integer $k$. For $V=V_{2 n}^{\delta}$ with $\delta \in\{ \pm 1\}$, put $V^{\prime}=V \oplus \mathbb{H}^{k}$. Also we set $V_{2 n+2 k}=\left(V_{2 n}\right)^{\prime}, W=W_{2 n}$ and $W^{\prime}=W_{2 n+2 k}=W \oplus \mathbb{H}^{k}$. Let $\phi_{\tau}$ be an irreducible orthogonal tempered representation of $W D_{F}$, and $\tau \in \operatorname{Irr}_{\text {temp }}\left(\operatorname{GL}_{k}(F)\right)$ be the corresponding representation. For $\phi_{\sigma} \in \Phi_{\text {temp }}^{\epsilon}\left(\mathrm{O}\left(V_{2 n}\right)\right)$, put

$$
\begin{aligned}
\phi_{\sigma^{\prime}} & =\phi_{\tau} \oplus \phi_{\sigma} \oplus \phi_{\tau} \in \Phi_{\text {temp }}^{\epsilon}\left(\mathrm{O}\left(V_{2 n+2 k}\right)\right), \\
\phi_{\pi} & =\left(\phi_{\sigma} \oplus \mathbf{1}\right) \otimes \chi_{V} \in \Phi_{\text {temp }}\left(\operatorname{Sp}\left(W_{2 n}\right)\right) \text { and } \\
\phi_{\pi^{\prime}} & =\left(\phi_{\sigma^{\prime}} \oplus \mathbf{1}\right) \otimes \chi_{V}=\phi_{\tau} \chi_{V} \oplus \phi_{\pi} \oplus \phi_{\tau} \chi_{V} \in \Phi_{\text {temp }}\left(\operatorname{Sp}\left(W_{2 n+2 k}\right)\right) .
\end{aligned}
$$

Let $\sigma \in \Pi_{\phi_{\sigma}}, \sigma^{\prime} \in \Pi_{\phi_{\sigma^{\prime}}}, \pi \in \Pi_{\phi_{\pi}}$ and $\pi^{\prime} \in \Pi_{\phi_{\pi^{\prime}}}$ such that $\sigma \in \operatorname{Irr}(\mathrm{O}(V))$, $\sigma^{\prime} \subset$ $\operatorname{Ind}_{P}^{\mathrm{O}\left(V^{\prime}\right)}(\tau \otimes \sigma)$ and $\pi^{\prime} \subset \operatorname{Ind}_{Q}^{\mathrm{Sp}\left(W^{\prime}\right)}\left(\tau \chi_{V} \otimes \pi\right)$, where $P \subset \mathrm{O}\left(V^{\prime}\right)$ and $Q \subset \operatorname{Sp}\left(W^{\prime}\right)$ 
are suitable parabolic subgroups. Suppose that

- $\phi_{\tau}$ is not the trivial representation of $W D_{F}$;

- $\pi^{\prime}=\theta_{W^{\prime}, V^{\prime}, \psi}\left(\sigma^{\prime}\right)$.

We denote by $a \in A_{\phi_{\sigma^{\prime}}}$ and $a^{\prime} \in A_{\phi_{\pi^{\prime}}}$ the elements corresponding to $\phi_{\tau}$ and $\phi_{\tau} \chi_{V}$, respectively. Then we have

$$
\iota_{c^{\prime}}\left(\sigma^{\prime}\right)(a)=\iota_{c^{\prime}}\left(\pi^{\prime}\right)\left(a^{\prime}\right)
$$

for $c^{\prime} \in F^{\times}$.

Proof. The argument is similar to those of [GI2, §8] and [At1, §7], but it has one difference. So we shall give a sketch of the proof.

Let $\omega=\omega_{W, V, \psi}$ and $\omega^{\prime}=\omega_{W^{\prime}, V^{\prime}, \psi}$. We use a mixed model $\mathcal{S}^{\prime}=\mathcal{S}\left(V^{\prime} \otimes Y^{*}\right) \otimes$ $\mathcal{S} \otimes \mathcal{S}\left(X^{*} \otimes W\right)$ for $\omega^{\prime}$, where $\mathcal{S}$ is a space of $\omega$ (cf., GI2, §7.4]). For $\varphi \in \mathcal{S}^{\prime}$, we define a map $\hat{f}(\varphi): \operatorname{Sp}\left(W^{\prime}\right) \times \mathrm{O}\left(V^{\prime}\right) \rightarrow \mathcal{S}$ as in [GI2, §8.1] and [At1, §7]. By a similar argument to the proof of [GS, Theorem 8.1], we have $\pi=\theta_{W, V, \psi}(\sigma)$ (see also [GI1, Proposition C.4]). Fix a non-zero $\mathrm{Sp}(W) \times \mathrm{O}(V)$-equivariant map

$$
\mathcal{T}_{00}: \omega \times \sigma^{\vee} \rightarrow \pi
$$

For $\varphi \in \mathcal{S}^{\prime}, \Phi_{s} \in \operatorname{Ind}_{P}^{\mathrm{O}\left(V^{\prime}\right)}\left(\tau|\cdot|_{F}^{s} \otimes \sigma^{\vee}\right), g \in \operatorname{Sp}\left(W^{\prime}\right), \check{v} \in \tau^{\vee}$ and $\check{v}_{0} \in \pi^{\vee}$, consider the integral

$$
\begin{aligned}
& \left\langle\mathcal{T}_{s}\left(\varphi, \Phi_{s}\right)(g), \check{v} \otimes \check{v_{0}}\right\rangle \\
& \quad:=L(s+1, \tau)^{-1} \cdot \int_{U_{P} \mathrm{O}(V) \backslash \mathrm{O}\left(V^{\prime}\right)}\left\langle\mathcal{T}_{00}\left(\hat{f}(\varphi)(g, h),\left\langle\Phi_{s}(h), \check{v}\right\rangle\right), \check{v}_{0}\right\rangle d h .
\end{aligned}
$$

Then one can show that

(1) the integral $\left\langle\mathcal{T}_{s}\left(\varphi, \Phi_{s}\right)(g), \check{v} \otimes \check{v_{0}}\right\rangle$ is absolutely convergent for $\operatorname{Re}(s)>-1$ and admits analytic continuation to $\mathbb{C}$;

(2) this integral gives an $\mathrm{Sp}\left(W^{\prime}\right) \times \mathrm{O}\left(V^{\prime}\right)$-equivalent map

$$
\mathcal{T}_{s}: \omega^{\prime} \otimes \operatorname{Ind}_{P}^{\mathrm{O}\left(V^{\prime}\right)}\left(\tau|\cdot|_{F}^{s} \otimes \sigma^{\vee}\right) \rightarrow \operatorname{Ind}_{Q}^{\mathrm{Sp}\left(W^{\prime}\right)}\left(\tau \chi_{V}|\cdot|_{F}^{S} \otimes \pi\right) .
$$

See [GI2, Lemmas 8.1-8.2] and At1, Proposition 7.2]. The one difference is that our case does not satisfy an analogue of GI2, Lemma 8.3]. So we have to modify this lemma. One can show that

(3) if $L\left(-s, \tau^{\vee}\right)$ is regular at $s=0$, then for any $\Phi \in \operatorname{Ind}_{P}^{\mathrm{O}\left(V^{\prime}\right)}\left(\tau \otimes \sigma^{\vee}\right)$ with $\Phi \neq 0$, there exists $\varphi \in \omega^{\prime}$ such that $\mathcal{T}_{0}\left(\varphi^{\prime}, \Phi\right) \neq 0$.

Since $\phi_{\tau}$ is irreducible and tempered, $L\left(-s, \tau^{\vee}\right)$ is regular at $s=0$ if and only if $\phi_{\tau}$ is not the trivial representation of $W D_{F}$.

By the same calculation as [GI2, Proposition 8.4] and [At1, Corollary 7.4], one can show that

(4) for $\Phi \in \operatorname{Ind}_{P}^{\mathrm{O}\left(V^{\prime}\right)}\left(\tau \otimes \sigma^{\vee}\right)$ and $\varphi \in \omega^{\prime}$, we have

$$
R\left(w^{\prime}, \tau \chi_{V} \otimes \pi\right) \mathcal{T}_{0}(\varphi, \Phi)=\omega_{\tau \chi_{V}}\left(c^{\prime}\right) \cdot \mathcal{T}_{0}\left(\varphi, R_{c^{\prime}}\left(w, \tau \otimes \sigma^{\vee}\right) \Phi\right) .
$$

Here, we use the fact that

$$
\gamma_{V}^{-1} \cdot \lambda(E / F, \psi)=e(V) \cdot \chi_{V}(c),
$$

where $\gamma_{V}$ is the Weil constant associated to $V$ which appears on the explicit formula for $\omega^{\prime}$, and $\lambda(E / F, \psi)$ is the Langlands constant which appears on the normalizing factor of $R_{c^{\prime}}\left(w, \tau \otimes \sigma^{\vee}\right)$. 
By the same argument as [At1, Lemma 7.5], (3) and (4) together with Hypothesis 3.13 and Proposition 3.16 imply that

(5) $\iota_{c^{\prime}}\left(\sigma^{\prime}\right)(a)=\omega_{\tau \chi_{V}}\left(c^{\prime}\right) \cdot \iota_{1}\left(\pi^{\prime}\right)\left(a^{\prime}\right)$.

Since $\omega_{\tau \chi_{V}}\left(c^{\prime}\right)=\operatorname{det}\left(\phi_{\tau} \chi_{V}\right)\left(c^{\prime}\right)=\operatorname{det}\left(\phi_{\pi^{\prime}}^{a^{\prime}}\right)\left(c^{\prime}\right)$, we have

(6) $\omega_{\tau \chi_{V}}\left(c^{\prime}\right) \cdot \iota_{1}\left(\pi^{\prime}\right)\left(a^{\prime}\right)=\iota_{c^{\prime}}\left(\pi^{\prime}\right)\left(a^{\prime}\right)$.

The equations (5) and (6) imply the desired equation.

Theorem 4.5 and Proposition 4.10 imply Prasad's conjecture (Theorem 4.4).

Proof of Theorem 4.4. By Remark 4.3 (2) and Lemmas 4.8 and 4.9, we only consider Conjecture $4.2(2)$ for $\phi_{\sigma} \in \Phi_{\text {temp }}^{\epsilon}(\mathrm{O}(V))$. Hence $\phi_{\sigma}$ contains an irreducible orthogonal representation $\phi_{0}$ with odd dimension $k_{0}$. Put $\phi_{\pi}=\left(\phi_{\sigma} \oplus \mathbf{1}\right) \otimes \chi_{V}$. Let $\sigma \in \Pi_{\phi_{\sigma}} \cap \operatorname{Irr}_{\text {temp }}\left(\mathrm{O}\left(V^{\delta}\right)\right)$ and assume that $\pi=\theta_{W, V^{\delta}, \psi}(\sigma)$ is non-zero. Hence we have $\pi \in \Pi_{\phi_{\pi}}$. Let $a_{0} \in A_{\phi_{\sigma}}$ (resp. $a_{0}^{\prime} \in A_{\phi_{\pi}}$ ) be the element corresponding to $\phi_{0}$ (resp. $\left.\phi_{0} \chi_{V}\right)$. Since $\left[A_{\phi_{\sigma}}: A_{\phi_{\sigma}}^{+}\right]=2$, by Theorem 4.5] it is enough to show that

$$
\iota_{c^{\prime}}(\sigma)\left(a_{0}\right)=\iota_{c^{\prime}}(\pi)\left(a_{0}^{\prime}\right)
$$

for $c^{\prime} \in F^{\times}$. We choose an irreducible orthogonal tempered representation $\phi_{\tau}$ of $W D_{F}$ such that

- $\phi_{\tau}$ is not the trivial representation;

- $\phi_{\tau}$ is not contained in $\phi_{\sigma}$;

- $k=\operatorname{dim}\left(\phi_{\tau}\right)$ is odd.

Put $\phi_{\sigma^{\prime}}=\phi_{\tau} \oplus \phi_{\sigma} \oplus \phi_{\tau}$ and $\phi_{\pi^{\prime}}=\phi_{\tau} \chi_{V} \oplus \phi_{\pi} \oplus \phi_{\tau} \chi_{V}$. Let $a_{\tau} \in A_{\phi_{\sigma^{\prime}}}$ (resp. $a_{\tau}^{\prime} \in$ $A_{\phi_{\pi^{\prime}}}$ ) be the element corresponding to $\phi_{\tau}$ (resp. $\phi_{\tau} \chi_{V}$ ). By the claims (2) and (3) in the proof of Proposition 4.10, there exist $\sigma^{\prime} \subset \operatorname{Ind}_{P}^{\mathrm{O}\left(V^{\delta^{\prime}}\right)}(\tau \otimes \sigma)$ and $\pi^{\prime} \subset$ $\operatorname{Ind}_{Q}^{\operatorname{Sp}\left(W^{\prime}\right)}\left(\tau \chi_{V} \otimes \pi\right)$ such that $\pi^{\prime}=\theta_{W^{\prime}, V^{\delta^{\prime}}, \psi}\left(\sigma^{\prime}\right)$. By Proposition 4.10, we have

$$
\iota_{c^{\prime}}\left(\sigma^{\prime}\right)\left(a_{\tau}\right)=\iota_{c^{\prime}}\left(\pi^{\prime}\right)\left(a_{\tau}^{\prime}\right) .
$$

On the other hand, we know

$$
\iota_{c^{\prime}}\left(\sigma^{\prime}\right)\left(a_{\tau}+a_{0}\right)=\iota_{c^{\prime}}\left(\pi^{\prime}\right)\left(a_{\tau}^{\prime}+a_{0}^{\prime}\right)
$$

by Theorem 4.5. Hence we have

$$
\iota_{c^{\prime}}\left(\sigma^{\prime}\right)\left(a_{0}\right)=\iota_{c^{\prime}}\left(\pi^{\prime}\right)\left(a_{0}^{\prime}\right) .
$$

Since $\iota_{c^{\prime}}\left(\sigma^{\prime}\right) \mid A_{\phi_{\sigma}}=\iota_{c^{\prime}}(\sigma)$ and $\iota_{c^{\prime}}\left(\pi^{\prime}\right) \mid A_{\phi_{\pi}}=\iota_{c^{\prime}}(\pi)$, we have

$$
\iota_{c^{\prime}}(\sigma)\left(a_{0}\right)=\iota_{c^{\prime}}(\pi)\left(a_{0}^{\prime}\right) \text {. }
$$

This completes the proof.

Remark 4.11. One may feel that Prasad's conjecture (Conjecture4.2) can be proven by a similar way to [At1, Theorem $1.7(\S 7)$ ] without assuming the weaker version of Prasad's conjecture (Theorem 4.5). However, because of the lack of an analogue of GI2, Lemma 8.3], the same method as At1 cannot be applied to Prasad's conjecture for $\left(\mathrm{O}\left(V_{2 n}\right), \operatorname{Sp}\left(W_{2 n}\right)\right)$ when $\phi \in \Phi_{\text {temp }}\left(\mathrm{O}\left(V_{2 n}\right)\right)$ contains 1 . 


\section{Gross-Prasad conjecture}

Gross and Prasad gave a conjectural answer for a restriction problem for special orthogonal groups. For the tempered case, this conjecture has been proven by Waldspurger W2, W3, W5, W6. In this section, we recall the Gross-Prasad conjecture and consider an analogous restriction problem for orthogonal groups.

5.1. Gross-Prasad conjecture for special orthogonal groups. In this subsection, we recall the Gross-Prasad conjecture.

Let $V_{m+1}=V_{m+1}^{+}$be an orthogonal space of dimension $m+1$, and $V_{m}=V_{m}^{+}$ be a non-degenerate subspace of $V_{m+1}$ with codimension 1 . We denote by $V_{\text {even }}$ (resp. $\left.V_{\text {odd }}\right)$ the space $V_{m}$ or $V_{m+1}$ such that $\operatorname{dim}\left(V_{\text {even }}\right)$ is even (resp. $\operatorname{dim}\left(V_{\text {odd }}\right)$ is odd). Suppose that $\mathrm{SO}\left(V_{m}\right) \times \mathrm{SO}\left(V_{m+1}\right)$ is quasi-split. We put

$$
c=-\operatorname{disc}\left(V_{\text {odd }}\right) / \operatorname{disc}\left(V_{\text {even }}\right) \in F^{\times} / F^{\times 2} .
$$

Then $V_{\text {even }}$ is associated to $\left(\operatorname{disc}\left(V_{\text {even }}\right), c\right)$. For $\delta, \delta^{\prime} \in\{ \pm 1\}$, we say that a pair $\left(V_{m}^{\delta}, V_{m+1}^{\delta^{\prime}}\right)$ is relevant if $V_{m}^{\delta} \subset V_{m+1}^{\delta^{\prime}}$. This is equivalent to $\delta=\delta^{\prime}$. In this case, we have a diagonal map

$$
\Delta: \mathrm{O}\left(V_{m}^{\delta}\right) \rightarrow \mathrm{O}\left(V_{m}^{\delta}\right) \times \mathrm{O}\left(V_{m+1}^{\delta}\right) .
$$

By [AGRS] and [W4, for $\sigma_{0} \in \operatorname{Irr}\left(\mathrm{SO}\left(V_{\text {even }}^{\delta}\right)\right)$ and $\tau_{0} \in \operatorname{Irr}\left(\mathrm{SO}\left(V_{\text {odd }}^{\delta}\right)\right)$, we have

$$
\operatorname{dim}_{\mathbb{C}} \operatorname{Hom}_{\Delta \mathrm{SO}\left(V_{m}^{\delta}\right)}\left(\sigma_{0} \otimes \tau_{0}, \mathbb{C}\right) \leq 1 .
$$

Choose $\epsilon \in \mathrm{O}\left(V_{m}^{\delta}\right)$ such that $\operatorname{det}(\epsilon)=-1$. We extend $\tau_{0}$ to an irreducible representation $\tau$ of $\mathrm{O}\left(V_{\text {odd }}^{\delta}\right)$. For $\varphi \in \operatorname{Hom}_{\Delta \mathrm{SO}\left(V_{m}^{\delta}\right)}\left(\sigma_{0} \otimes \tau_{0}, \mathbb{C}\right)$, we put

$$
\varphi^{\prime}=\varphi \circ(1 \otimes \tau(\epsilon)) .
$$

Then we have $\varphi^{\prime} \in \operatorname{Hom}_{\Delta \mathrm{SO}\left(V_{m}^{\delta}\right)}\left(\sigma_{0}^{\epsilon} \otimes \tau_{0}, \mathbb{C}\right)$, and the map $\varphi \mapsto \varphi^{\prime}$ gives an isomorphism

$$
\operatorname{Hom}_{\Delta \mathrm{SO}\left(V_{m}^{\delta}\right)}\left(\sigma_{0} \otimes \tau_{0}, \mathbb{C}\right) \cong \operatorname{Hom}_{\Delta \mathrm{SO}\left(V_{m}^{\delta}\right)}\left(\sigma_{0}^{\epsilon} \otimes \tau_{0}, \mathbb{C}\right) .
$$

Therefore, $\operatorname{dim}_{\mathbb{C}} \operatorname{Hom}_{\Delta \mathrm{SO}\left(V_{m}^{\delta}\right)}\left(\sigma_{0} \otimes \tau_{0}, \mathbb{C}\right)$ depends only on

$$
\left(\left[\sigma_{0}\right], \tau_{0}\right) \in \operatorname{Irr}\left(\mathrm{SO}\left(V_{\text {even }}^{\delta}\right)\right) / \sim_{\epsilon} \times \operatorname{Irr}\left(\mathrm{SO}\left(V_{\text {odd }}^{\delta}\right)\right) .
$$

The Gross-Prasad conjecture determines this dimension in terms of Weak LLC for $\mathrm{SO}\left(V_{\text {even }}\right)$ and LLC for $\mathrm{SO}\left(V_{\text {odd }}\right)$.

Let $\phi \in \Phi_{\text {temp }}\left(\mathrm{SO}\left(V_{\text {even }}\right)\right) / \sim_{\epsilon}$ and $\phi^{\prime} \in \Phi_{\text {temp }}\left(\mathrm{SO}\left(V_{\text {odd }}\right)\right)$. Following [GGP, $\left.\$ 6\right]$, for semi-simple elements $a \in C_{\phi}$ and $a^{\prime} \in C_{\phi^{\prime}}$, we put

$$
\begin{aligned}
& \chi_{\phi^{\prime}}(a)=\varepsilon\left(\phi^{a} \otimes \phi^{\prime}\right) \cdot \operatorname{det}\left(\phi^{a}\right)(-1)^{\frac{1}{2} \operatorname{dim}\left(\phi^{\prime}\right),} \\
& \chi_{\phi}\left(a^{\prime}\right)=\varepsilon\left(\phi \otimes \phi^{\prime a^{\prime}}\right) \cdot \operatorname{det}(\phi)(-1)^{\frac{1}{2} \operatorname{dim}\left(\phi^{\prime a^{\prime}}\right) .}
\end{aligned}
$$

Here, $\varepsilon\left(\phi^{a} \otimes \phi^{\prime}\right)=\varepsilon\left(1 / 2, \phi^{a} \otimes \phi^{\prime}, \psi\right)$ and $\varepsilon\left(\phi \otimes \phi^{\prime a^{\prime}}\right)=\varepsilon\left(1 / 2, \phi \otimes \phi^{\prime a^{\prime}}, \psi\right)$ are the local root numbers, which are independent of the choice of $\psi$. By [GP, Proposition 10.5], $\chi_{\phi^{\prime}}$ and $\chi_{\phi}$ define characters on $A_{\phi}$ and on $A_{\phi^{\prime}}$, respectively. Note that $\chi_{\phi^{\prime}}\left(z_{\phi}\right)=\chi_{\phi}\left(z_{\phi^{\prime}}\right)$.

The following is a result of Waldspurger [W2, W3, W5, W6.

Theorem 5.1 (Gross-Prasad conjecture for special orthogonal groups). Let $V_{m+1}$ $=V_{m+1}^{+}$be an orthogonal space of dimension $m+1$, and $V_{m}=V_{m}^{+}$be a nondegenerate subspace of $V_{m+1}$ with codimension 1 . Suppose that $\mathrm{SO}\left(V_{m}\right) \times \mathrm{SO}\left(V_{m+1}\right)$ 
is quasi-split. We put $c=-\operatorname{disc}\left(V_{\text {odd }}\right) / \operatorname{disc}\left(V_{\text {even }}\right) \in F^{\times} / F^{\times 2}$, so that $V_{\text {even }}$ is associated to $\left(\operatorname{disc}\left(V_{\text {even }}\right), c\right)$. Assume

- Weak LLC for $\mathrm{SO}\left(V_{\text {even }}\right)$ (Desideratum 3.5);

- $L L C$ for $\mathrm{SO}\left(V_{\text {odd }}\right)$ (Desideratum 3.1 for $\left.\mathrm{SO}\left(V_{\text {odd }}\right)\right)$.

Let $\phi \in \Phi_{\text {temp }}\left(\mathrm{SO}\left(V_{\text {even }}\right)\right) / \sim_{\epsilon}$ and $\phi^{\prime} \in \Phi_{\text {temp }}\left(\mathrm{SO}\left(V_{\text {odd }}\right)\right)$. Then there exists a unique pair $\left(\left[\sigma_{0}\right], \tau_{0}\right) \in \Pi_{\phi}^{0} \times \Pi_{\phi^{\prime}}$ such that $\sigma_{0} \otimes \tau_{0}$ is a representation of $\mathrm{SO}\left(V_{\text {even }}^{\delta}\right) \times$ $\mathrm{SO}\left(V_{\text {odd }}^{\delta}\right)$ for some $\delta \in\{ \pm 1\}$, and

$$
\operatorname{Hom}_{\Delta \mathrm{SO}\left(V_{2 n}^{\delta}\right)}\left(\sigma_{0} \otimes \tau_{0}, \mathbb{C}\right) \neq 0 .
$$

Moreover, $\iota_{c}\left(\left[\sigma_{0}\right]\right) \times \iota\left(\tau_{0}\right)$ satisfies that

$$
\iota_{c}\left(\left[\sigma_{0}\right]\right) \times \iota\left(\tau_{0}\right)=\left(\chi_{\phi^{\prime}} \mid A_{\phi}^{+}\right) \times \chi_{\phi} .
$$

5.2. Gross-Prasad conjecture for orthogonal groups. Let $V_{m+1}^{\delta}$ be an orthogonal space of dimension $m+1$, and $V_{m}^{\delta}$ be a non-degenerate subspace of $V_{m+1}^{\delta}$ with codimension 1. In AGRS, Aizenbud, Gourevitch, Rallis and Schiffmann showed that

$$
\operatorname{dim}_{\mathbb{C}} \operatorname{Hom}_{\Delta \mathrm{O}\left(V_{m}^{\delta}\right)}(\sigma \otimes \tau, \mathbb{C}) \leq 1
$$

for $\sigma \in \operatorname{Irr}\left(\mathrm{O}\left(V_{\text {even }}^{\delta}\right)\right)$ and $\tau \in \operatorname{Irr}\left(\mathrm{O}\left(V_{\text {odd }}^{\delta}\right)\right)$. The following conjecture determines this dimension for $(\sigma, \tau) \in \operatorname{Irr}_{\text {temp }}\left(\mathrm{O}\left(V_{\text {even }}^{\delta}\right)\right) \times \operatorname{Irr}_{\text {temp }}\left(\mathrm{O}\left(V_{\text {odd }}^{\delta}\right)\right)$.

Let $\phi \in \Phi\left(\mathrm{O}\left(V_{2 n}\right)\right)$. For $b \in\{ \pm 1\}$ and $a \in C_{\phi}$, we put

$$
d_{\phi, b}(a)=b^{\operatorname{dim}\left(\phi^{a}\right)} .
$$

By [GGP $\S 4], d_{\phi, b}$ defines a character on $A_{\phi}$. Note that $d_{\phi, b}$ is trivial on $A_{\phi}^{+}$.

Conjecture 5.2 (Gross-Prasad conjecture for orthogonal groups). Let $V_{m+1}=$ $V_{m+1}^{+}$be an orthogonal space of dimension $m+1$, and $V_{m}=V_{m}^{+}$be a non-degenerate subspace of $V_{m+1}$ with codimension 1 . Suppose that $\mathrm{O}\left(V_{m}\right) \times \mathrm{O}\left(V_{m+1}\right)$ is quasisplit. We put $c=-\operatorname{disc}\left(V_{\text {odd }}\right) / \operatorname{disc}\left(V_{\text {even }}\right) \in F^{\times} / F^{\times 2}$, so that $V_{\text {even }}$ is associated to $\left(\operatorname{disc}\left(V_{\text {even }}\right), c\right)$. Let $\phi \in \Phi_{\text {temp }}\left(\mathrm{O}\left(V_{\text {even }}\right)\right)$ and $\left(\phi^{\prime}, b\right) \in \Phi_{\text {temp }}\left(\mathrm{O}\left(V_{\text {odd }}\right)\right)$. Then there exists a unique pair $(\sigma, \tau) \in \Pi_{\phi} \times \Pi_{\phi^{\prime}, b}$ such that $\sigma \otimes \tau$ is a representation of $\mathrm{O}\left(V_{\text {even }}^{\delta}\right) \times \mathrm{O}\left(V_{\text {odd }}^{\delta}\right)$ for some $\delta \in\{ \pm 1\}$, and

$$
\operatorname{Hom}_{\Delta \mathrm{O}\left(V_{m}^{\delta}\right)}(\sigma \otimes \tau, \mathbb{C}) \neq 0 .
$$

Moreover, $\iota_{c}(\sigma) \times \iota(\tau)$ satisfies that

$$
\iota_{c}(\sigma) \times \iota(\tau)=\left(\chi_{\phi^{\prime}} \cdot d_{\phi, b}\right) \times \chi_{\phi} .
$$

Remark 5.3. Let $V_{2 n+1}=V_{2 n} \oplus L$ be an orthogonal space of dimension $2 n+1$, and $V_{2 n}$ be a non-degenerate subspace of $V_{2 n+1}$ with codimension 1 . The stabilizer of the line $L$ in $\mathrm{SO}\left(V_{2 n+1}\right)$ is the subgroup:

$$
\mathrm{S}\left(\mathrm{O}\left(V_{2 n}\right) \times \mathrm{O}(L)\right)=\left\{\left(g_{1}, g_{2}\right) \in \mathrm{O}\left(V_{2 n}\right) \times \mathrm{O}(L) \mid \operatorname{det}\left(g_{1}\right)=\operatorname{det}\left(g_{2}\right)\right\},
$$

which is isomorphic to $\mathrm{O}\left(V_{2 n}\right)$ by the first projection. Then the restriction problem of $\mathrm{O}\left(V_{2 n}\right) \subset \mathrm{O}\left(V_{2 n+1}\right)$ is equivalent to the one of $\mathrm{S}\left(\mathrm{O}\left(V_{2 n}\right) \times \mathrm{O}(L)\right) \subset \mathrm{SO}\left(V_{2 n+1}\right)$. Indeed, let $\tau$ be an irreducible representation of $\mathrm{SO}\left(V_{2 n+1}\right)$, and $\tau^{b}$ be the extension of $\tau$ to $\mathrm{O}\left(V_{2 n+1}\right)$ satisfying $\tau^{b}\left(-\mathbf{1}_{V_{2 n+1}}\right)=b \cdot$ id for $b \in\{ \pm 1\}$. For $\sigma \in \operatorname{Irr}\left(\mathrm{O}\left(V_{2 n}\right)\right)$, define $\sigma^{b} \in \operatorname{Irr}\left(\mathrm{O}\left(V_{2 n}\right)\right)$ by

$$
\sigma^{b}(g)= \begin{cases}\sigma(g) & \text { if } \operatorname{det}(g)=1 \\ b \cdot \omega_{\sigma}(-1) \cdot \sigma(g) & \text { if } \operatorname{det}(g)=-1\end{cases}
$$


Here, $\omega_{\sigma}$ denotes the central character of $\sigma$, which is regarded as a character of $\{ \pm 1\}$. We regard $\sigma^{b}$ as an irreducible representation of $\mathrm{S}\left(\mathrm{O}\left(V_{2 n}\right) \times \mathrm{O}(L)\right)$ by pulling back via the first projection. Then we have an identification

$$
\operatorname{Hom}_{\mathrm{O}\left(V_{2 n}\right)}\left(\tau^{b} \otimes \sigma, \mathbb{C}\right)=\operatorname{Hom}_{\mathrm{S}\left(\mathrm{O}\left(V_{2 n}\right) \times \mathrm{O}(L)\right)}\left(\tau \otimes \sigma^{b}, \mathbb{C}\right) .
$$

Using this equation, we see that a result of Prasad ([P3, Theorem 4]) follows from Conjecture 5.2 for $m=2$.

In $\$ 5.3$, we review another result of Prasad [P3] for a low rank case and check that it is compatible with Conjecture 5.2. In \$5.4 we will prove Conjecture 5.2 provided LLC for $\mathrm{O}\left(V_{m}\right) \times \mathrm{O}\left(V_{m+1}\right)$ and Hypothesis 3.13 hold.

5.3. Low rank cases. In [P3], D. Prasad extended a theorem on trilinear forms of three representations of $\mathrm{GL}_{2}(F)([\mathrm{P} 1$, Theorem 1.4]). In this subsection, we check this theorem follows from Conjecture 5.2 .

First, we recall a theorem on trilinear forms. Let $D$ be the (unique) quaternion division algebra over $F$. For an irreducible representation $\pi$ of $\mathrm{GL}_{2}(F)$, let $\pi^{\prime}$ be the Jacquet-Langlands lift of $\pi$ if $\pi$ is an essentially discrete series representation, and put $\pi^{\prime}=0$ otherwise. Also, for a representation $\phi$ of $W D_{F}$, if $\operatorname{det}(\phi)=\mathbf{1}$, we write $\varepsilon(\phi)=\varepsilon(1 / 2, \phi, \psi)$, which is independent of a non-trivial additive character $\psi$ of $F$.

Theorem 5.4 ([P1, Theorem 1.4]). For $i=1,2,3$, let $\pi_{i}$ be an irreducible infinitedimensional representation of $\mathrm{GL}_{2}(F)$ with central character $\omega_{\pi_{i}}$. Assume that $\omega_{\pi_{1}} \omega_{\pi_{2}} \omega_{\pi_{3}}=\mathbf{1}$. We denote the representation of $W D_{F}$ corresponding to $\pi_{i}$ by $\phi_{i}$. Then:

- there exists a non-zero $\mathrm{GL}_{2}(F)$-invariant linear form on $\pi_{1} \otimes \pi_{2} \otimes \pi_{3}$ if and only if $\varepsilon\left(\phi_{1} \otimes \phi_{2} \otimes \phi_{3}\right)=1$;

- there exists a non-zero $D^{\times}$-invariant linear form on $\pi_{1}^{\prime} \otimes \pi_{2}^{\prime} \otimes \pi_{3}^{\prime}$ if and only if $\varepsilon\left(\phi_{1} \otimes \phi_{2} \otimes \phi_{3}\right)=-1$.

When $\pi_{i}$ is tempered for each $i$ and $\omega_{\pi_{1}} \omega_{\pi_{2}}=\omega_{\pi_{3}}=\mathbf{1}$, this theorem is a special case of GP conjecture for special orthogonal groups (Theorem 5.1). Recall that there exist two exact sequences

where

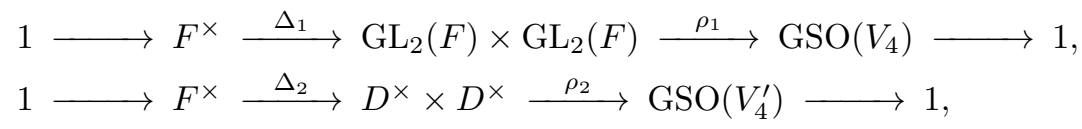

- $V_{4}=\mathrm{M}_{2}(F)$ which is regarded as an orthogonal space with

$$
\left\langle\left(\begin{array}{l}
a_{1}, b_{1} \\
c_{1}, d_{1}
\end{array}\right),\left(\begin{array}{l}
a_{2}, b_{2} \\
c_{2}, d_{2}
\end{array}\right)\right\rangle_{V_{4}}=\operatorname{tr}\left(\left(\begin{array}{c}
a_{1}, b_{1} \\
c_{1}, d_{1}
\end{array}\right)\left(\begin{array}{l}
d_{2},-b_{2} \\
-c_{2}, a_{2}
\end{array}\right)\right) ;
$$

- $V_{4}^{\prime}=D$ is regarded as an orthogonal space with

$$
\langle x, y\rangle_{V_{4}^{\prime}}=\tau\left(x y^{*}\right)
$$

where $y \mapsto y^{*}$ is the main involution and $\tau(x)$ is the reduced trace of $x$;

- for an orthogonal space $V_{2 n}$ with even dimension $2 n$, the orthogonal similitude group $\mathrm{GO}\left(V_{2 n}\right)$ is defined by

$$
\begin{aligned}
\mathrm{GO}\left(V_{2 n}\right) & =\left\{g \in \mathrm{GL}\left(V_{2 n}\right) \mid\left\langle g v_{1}, g v_{2}\right\rangle_{V_{2 n}}\right. \\
& \left.=\nu(g)\left\langle v_{1}, v_{2}\right\rangle_{V_{2 n}}, \nu(g) \in F^{\times} \text {for any } v_{1}, v_{2} \in V_{2 n}\right\}
\end{aligned}
$$


and $\operatorname{GSO}\left(V_{2 n}\right)$ is defined by

$$
\operatorname{GSO}\left(V_{2 n}\right)=\left\{g \in \operatorname{GO}\left(V_{2 n}\right) \mid \operatorname{det}(g)=\nu(g)^{n}\right\} ;
$$

- $\Delta_{1}$ and $\Delta_{2}$ are the diagonal embeddings;

- $\rho_{1}$ and $\rho_{2}$ are given by

$$
\rho_{1}\left(g_{1}, g_{2}\right) x=g_{1} x g_{2}^{-1}, \quad \rho_{2}\left(g_{1}^{\prime}, g_{2}^{\prime}\right) x^{\prime}=g_{1}^{\prime} x^{\prime} g_{2}^{\prime-1}
$$

for $g_{1}, g_{2} \in \mathrm{GL}_{2}(F), x \in V_{4}, g_{1}^{\prime}, g_{2}^{\prime} \in D^{\times}$and $x^{\prime} \in V_{4}^{\prime}$.

Hence if $\omega_{\pi_{1}} \omega_{\pi_{2}}=\omega_{\pi_{3}}=\mathbf{1}$, then $\pi_{1} \otimes \pi_{2}$ (resp. $\pi_{1}^{\prime} \otimes \pi_{2}^{\prime}$ ) is regarded as a representation $\widetilde{\sigma}$ of $\operatorname{GSO}\left(V_{4}\right)$ (resp. $\widetilde{\sigma}^{\prime}$ of $\operatorname{GSO}\left(V_{4}^{\prime}\right)$ ). The restriction of $\widetilde{\sigma}$ to $\mathrm{SO}\left(V_{4}\right)$ (resp. $\tilde{\sigma}^{\prime}$ to $\left.\mathrm{SO}\left(V_{4}^{\prime}\right)\right)$ decomposes into a direct sum of irreducible representations, i.e.,

$$
\tilde{\sigma} \mid \mathrm{SO}\left(V_{4}\right)=\sigma_{1} \oplus \cdots \oplus \sigma_{r} \quad\left(\operatorname{resp} . \tilde{\sigma}^{\prime} \mid \mathrm{SO}\left(V_{4}^{\prime}\right)=\sigma_{1}^{\prime} \oplus \cdots \oplus \sigma_{r^{\prime}}^{\prime}\right) .
$$

Let $\phi_{\sigma}=\phi_{1} \otimes \phi_{2}$. Then we have $\phi_{\sigma} \in \Phi\left(\mathrm{SO}\left(V_{4}\right)\right) / \sim_{\epsilon}$, and the $L$-packet $\Pi_{\phi_{\sigma}}^{0}$ is given by

$$
\Pi_{\phi_{\sigma}}^{0}=\left\{\left[\sigma_{i}\right] \mid i=1, \ldots, r\right\} \cup\left\{\left[\sigma_{i}^{\prime}\right] \mid i=1, \ldots, r^{\prime}\right\} .
$$

Here, if $\pi_{1}^{\prime} \otimes \pi_{2}^{\prime}=0$, we neglect $\left\{\left[\sigma_{i}^{\prime}\right]\right\}$.

On the other hand, if $\omega_{\pi_{3}}=\mathbf{1}$, then $\pi_{3}$ (resp. $\pi_{3}^{\prime}$ ) is regarded as a representation $\tau$ of $\mathrm{SO}\left(V_{3}\right)$ (resp. $\tau^{\prime}$ of $\mathrm{SO}\left(V_{3}^{\prime}\right)$ ), where $V_{3}$ (resp. $V_{3}^{\prime}$ ) is the orthogonal space of dimension 3, discriminant -1 , and such that $\mathrm{SO}\left(V_{3}\right)$ is split (resp. $\mathrm{SO}\left(V_{3}^{\prime}\right)$ is not split). We identify $V_{3}$ (resp. $V_{3}^{\prime}$ ) with the subspace of $V_{4}$ (resp. $V_{4}^{\prime}$ ) consisting of trace zero elements (resp. reduced trace zero elements). Let $\phi_{\tau}=\phi_{3}: W D_{F} \rightarrow \mathrm{SL}_{2}(\mathbb{C})$. Then we have $\phi_{\tau} \in \Phi\left(\mathrm{SO}\left(V_{3}\right)\right)$, and the $L$-packet $\Pi_{\phi_{\tau}}$ is given by

$$
\Pi_{\phi_{\tau}}=\left\{\tau, \tau^{\prime}\right\}
$$

Here, if $\pi_{3}^{\prime}=0$, we neglect $\tau^{\prime}$.

We embed $\mathrm{GL}_{2}(F)$ into $\mathrm{GL}_{2}(F) \times \mathrm{GL}_{2}(F)$ (resp. $D^{\times}$into $D^{\times} \times D^{\times}$) as the diagonal subgroup. This embedding induces the inclusion $\mathrm{SO}\left(V_{3}\right) \hookrightarrow \mathrm{SO}\left(V_{4}\right)$ (resp. $\mathrm{SO}\left(V_{3}^{\prime}\right) \hookrightarrow \mathrm{SO}\left(V_{4}^{\prime}\right)$ ). Then we conclude that

- there exists a non-zero $\mathrm{GL}_{2}(F)$-invariant linear form on $\pi_{1} \otimes \pi_{2} \otimes \pi_{3}$ if and only if $\operatorname{Hom}_{\mathrm{SO}\left(V_{3}\right)}\left(\sigma_{i} \otimes \tau, \mathbb{C}\right) \neq 0$ for some $i=1, \ldots, r$;

- there exists a non-zero $D^{\times}$-invariant linear form on $\pi_{1}^{\prime} \otimes \pi_{2}^{\prime} \otimes \pi_{3}^{\prime}$ if and only if $\operatorname{Hom}_{\mathrm{SO}\left(V_{3}^{\prime}\right)}\left(\sigma_{i}^{\prime} \otimes \tau^{\prime}, \mathbb{C}\right) \neq 0$ for some $i=1, \ldots, r^{\prime}$

- $\varepsilon\left(\phi_{1} \otimes \phi_{2} \otimes \phi_{3}\right)=\varepsilon\left(\phi_{\sigma} \otimes \phi_{\tau}\right)=\chi_{\phi_{\tau}}\left(z_{\phi_{\sigma}}\right)=\chi_{\phi_{\sigma}}\left(z_{\phi_{\tau}}\right)$.

Hence Theorem 5.1 implies Theorem 5.4 for tempered $\pi_{1}, \pi_{2}$ and $\pi_{3}$ when $\omega_{\pi_{1}} \omega_{\pi_{2}}=$ $\omega_{\pi_{3}}=1$.

Next, we recall [P3, Theorem 3], which is an extension of Theorem [5.4, This is the case when $\pi_{1}=\pi_{2}$. Note that $\pi_{1} \otimes \pi_{1}=\operatorname{Sym}^{2}\left(\pi_{1}\right) \oplus \wedge^{2}\left(\pi_{1}\right)$. Also, for a representation $\phi_{1}$ of $W D_{F}$, we have $\phi_{1} \otimes \phi_{1}=\operatorname{Sym}^{2}\left(\phi_{1}\right) \oplus \wedge^{2}\left(\phi_{1}\right)$.

Theorem 5.5 ([3, Theorem 3]). Let $\pi_{1}$ and $\pi_{3}$ be irreducible admissible infinitedimensional representations of $\mathrm{GL}_{2}(F)$. Assume that $\omega_{\pi_{1}}^{2} \omega_{\pi_{3}}=\mathbf{1}$. We denote the representation of $W D_{F}$ corresponding to $\pi_{i}$ by $\phi_{i}$. Then:

- $\operatorname{Sym}^{2}\left(\pi_{1}\right) \otimes \pi_{3}$ has a $\mathrm{GL}_{2}(F)$-invariant linear form if and only if $\varepsilon\left(\operatorname{Sym}^{2}\left(\phi_{1}\right)\right.$ $\left.\otimes \phi_{3}\right)=\omega_{\pi_{1}}(-1)$ and $\varepsilon\left(\wedge^{2}\left(\phi_{1}\right) \otimes \phi_{3}\right)=\omega_{\pi_{1}}(-1)$;

- $\wedge^{2}\left(\pi_{1}\right) \otimes \pi_{3}$ has a $\mathrm{GL}_{2}(F)$-invariant linear form if and only if $\varepsilon\left(\operatorname{Sym}^{2}\left(\phi_{1}\right) \otimes\right.$ $\left.\phi_{3}\right)=-\omega_{\pi_{1}}(-1)$ and $\varepsilon\left(\wedge^{2}\left(\phi_{1}\right) \otimes \phi_{3}\right)=-\omega_{\pi_{1}}(-1)$. 
We check that the GP conjecture (Conjecture 5.2) implies this theorem for tempered $\pi_{1}$ and $\pi_{3}$ such that $\omega_{\pi_{1}}^{2}=\mathbf{1}$ and $\omega_{\pi_{3}}=\mathbf{1}$. Consider the group

$$
\left(\mathrm{GL}_{2}(F) \times \mathrm{GL}_{2}(F)\right) \rtimes\langle c\rangle,
$$

where $c^{2}=1$ and $c$ acts on $\mathrm{GL}_{2}(F) \times \mathrm{GL}_{2}(F)$ by exchanging the two factors of $\mathrm{GL}_{2}(F)$. Then $\rho_{1}: \mathrm{GL}_{2}(F) \times \mathrm{GL}_{2}(F) \rightarrow \mathrm{GSO}\left(V_{4}\right)$ gives a surjection

$$
\rho_{1}:\left(\mathrm{GL}_{2}(F) \times \mathrm{GL}_{2}(F)\right) \rtimes\langle c\rangle \rightarrow \mathrm{GSO}\left(V_{4}\right) \rtimes\langle c\rangle \cong \mathrm{GO}\left(V_{4}\right) .
$$

Here, we identify $c$ as the element in $\mathrm{O}\left(V_{4}\right)$ which acts on $V_{3}$ by -1 and on the orthogonal complement of $V_{3}$ by +1 . There are two extensions of the representation $\widetilde{\sigma} \mid \mathrm{SO}\left(V_{4}\right)=\sigma_{1} \oplus \cdots \oplus \sigma_{r}$ of $\mathrm{SO}\left(V_{4}\right)$ on $\pi_{1} \otimes \pi_{1}=\operatorname{Sym}^{2}\left(\pi_{1}\right) \oplus \wedge^{2}\left(\pi_{1}\right)$ to $\mathrm{O}\left(V_{4}\right)$. We denote by $\widetilde{\sigma}^{ \pm} \mid \mathrm{O}\left(V_{4}\right)=\sigma_{1}^{ \pm} \oplus \cdots \oplus \sigma_{r}^{ \pm}$the extension such that $c$ acts on $\operatorname{Sym}^{2}\left(\pi_{1}\right)$ by \pm 1 and on $\wedge^{2}\left(\pi_{1}\right)$ by $\mp 1$, respectively. On the other hand, the representation $\tau$ of $\mathrm{SO}\left(V_{3}\right)$ on $\pi_{3}$ has two extensions $\tau^{ \pm}$to $\mathrm{O}\left(V_{3}\right)$, which satisfies that $\tau^{ \pm}\left(-\mathbf{1}_{V_{3}}\right)= \pm$ id. Since $c$ centralizes the diagonal subgroup $\mathrm{GL}_{2}(F)$ of $\mathrm{GL}_{2}(F) \times \mathrm{GL}_{2}(F)$, we see that

- $\operatorname{Sym}^{2}\left(\pi_{1}\right) \otimes \pi_{3}$ has a $\mathrm{GL}_{2}(F)$-invariant linear form if and only if

$$
\operatorname{Hom}_{\mathrm{O}\left(V_{3}\right)}\left(\sigma_{i}^{+} \otimes \tau^{+}, \mathbb{C}\right) \neq 0
$$

for some $i=1, \ldots, r$;

- $\wedge^{2}\left(\pi_{1}\right) \otimes \pi_{3}$ has a $\mathrm{GL}_{2}(F)$-invariant linear form if and only if

$$
\operatorname{Hom}_{\mathrm{O}\left(V_{3}\right)}\left(\sigma_{i}^{+} \otimes \tau^{-}, \mathbb{C}\right) \neq 0
$$

for some $i=1, \ldots, r$.

Since $\pi_{1}$ is generic, $\sigma_{i}^{ \pm}$is $\mu_{a}^{b}$-generic for some $a \in F^{\times}$and $b \in\{ \pm\}$. We claim that $b= \pm \omega_{\pi_{1}}(a)$. Fix a non-zero $\psi$-Whittaker functional $l: \pi_{1} \rightarrow \mathbb{C}$ and $x \in \pi_{1}$ such that $l(x) \neq 0$. For each $a \in F^{\times}$, put

$$
l_{a}=l \circ \pi_{1}\left(\left(\begin{array}{ll}
a & 0 \\
0 & 1
\end{array}\right)\right), \quad x_{a}=\pi_{1}\left(\left(\begin{array}{ll}
a & 0 \\
0 & 1
\end{array}\right)^{-1}\right) x .
$$

We define a basis $\left\{v, e_{a}, e_{a}^{\prime}, v^{\prime}\right\}$ of $V_{4}=\mathrm{M}_{2}(F)$ by

$$
v=\left(\begin{array}{ll}
0 & 1 \\
0 & 0
\end{array}\right), e_{a}=\left(\begin{array}{cc}
1 & 0 \\
0 & a
\end{array}\right), e_{a}^{\prime}=\left(\begin{array}{cc}
1 & 0 \\
0 & -a
\end{array}\right), v=\left(\begin{array}{cc}
0 & 0 \\
-1 & 0
\end{array}\right) .
$$

This basis makes $V_{4}$ the orthogonal space associated to $(1, a)$. Also we have

$$
\begin{aligned}
\rho_{1}\left(\left(\begin{array}{cc}
1 & n_{1} \\
0 & 1
\end{array}\right),\left(\begin{array}{cc}
1 & n_{2} \\
0 & 1
\end{array}\right)\right)\left(v, e_{a}, e_{a}^{\prime}, v^{\prime}\right) \\
\quad=\left(v, e_{a}, e_{a}^{\prime}, v^{\prime}\right)\left(\begin{array}{cccc}
1 & n_{1} a-n_{2} & -n_{1} a-n 2 & -n_{1} n_{2} \\
0 & 1 & 0 & \frac{-n_{1}+n_{2} a^{-1}}{2} \\
0 & 0 & 1 & \frac{-n_{1}-n_{2} a^{-1}}{2} \\
0 & 0 & 0 & 1
\end{array}\right) .
\end{aligned}
$$

Hence $l_{a} \otimes l_{-1}: \pi_{1} \otimes \pi_{1} \rightarrow \mathbb{C}$ gives a $\mu_{a}$-Whittaker functional $l_{0}$ on $\widetilde{\sigma}^{ \pm}$. Let $v_{0}=$ $x_{a} \otimes x_{-1} \in \tilde{\sigma}=\pi_{1} \otimes \pi_{1}$. Note that $V_{3}$ is the orthogonal complement of $F e_{1}$ in $V_{4}$. Since we regard $c$ as the non-trivial element in the center of $\mathrm{O}\left(V_{3}\right)$, it acts on $v, e_{1}$, $e_{1}^{\prime}$, and $v^{\prime}$ by

$$
c v=-v, c e_{1}=e_{1}, c e_{1}^{\prime}=-e_{1}^{\prime}, c v^{\prime}=-v^{\prime} .
$$

We define $\epsilon_{a} \in \mathrm{O}\left(V_{4}\right)$ so that $\epsilon_{a} v=v, \epsilon_{a} e_{a}=e_{a}, \epsilon_{a} e_{a}^{\prime}=-e_{a}^{\prime}$, and $\epsilon_{a} v^{\prime}=v^{\prime}$. Then

$$
\epsilon_{a}=\rho_{1}\left(\left(\begin{array}{cc}
-1 & 0 \\
0 & a
\end{array}\right),\left(\begin{array}{cc}
-a & 0 \\
0 & 1
\end{array}\right)\right) \cdot c .
$$


Therefore we have

$$
\begin{aligned}
b \cdot l_{0}\left(v_{0}\right) & =l_{0}\left(\epsilon_{a} v_{0}\right) \\
& =\left(l_{a} \otimes l_{-1}\right) \circ \pi_{1}\left(\left(\begin{array}{cc}
-1 & 0 \\
0 & a
\end{array}\right)\right) \otimes \pi_{1}\left(\left(\begin{array}{cc}
-a & 0 \\
0 & 1
\end{array}\right)\right) \circ c\left(x_{a} \otimes x_{-1}\right) \\
& = \pm \omega_{\pi_{1}}(a) \cdot\left(l_{a} \otimes l_{-1}\right) \circ \pi_{1}\left(\left(\begin{array}{cc}
-a^{-1} & 0 \\
0 & 1
\end{array}\right)\right) \otimes \pi_{1}\left(\left(\begin{array}{cc}
-a & 0 \\
0 & 1
\end{array}\right)\right)\left(x_{-1} \otimes x_{a}\right) \\
& = \pm \omega_{\pi_{1}}(a) \cdot\left(l_{a} \otimes l_{-1}\right)\left(x_{a} \otimes x_{-1}\right) \\
& = \pm \omega_{\pi_{1}}(a) \cdot l_{0}\left(v_{0}\right) .
\end{aligned}
$$

Hence $b= \pm \omega_{\pi_{1}}(a)$, as desired.

By Desideratum 3.12 (1) and (3), we have

$$
\iota_{1}\left(\sigma_{i}^{ \pm}\right)(s)=\left( \pm \omega_{\pi_{1}}(a)\right)^{\operatorname{det}(s)} \cdot \eta_{\phi_{\sigma}, a}(s)
$$

for $s \in A_{\phi_{\sigma}}$, In particular, if we denote the element in $A_{\phi_{\sigma}}$ corresponding to $\wedge^{2}\left(\phi_{1}\right)=\operatorname{det}\left(\phi_{1}\right)=\omega_{\pi_{1}}$ by $s_{0}$, we have

$$
\iota_{1}\left(\sigma_{i}^{ \pm}\right)\left(s_{0}\right)= \pm 1 \text {. }
$$

Also we have

$$
\left(\chi_{\phi_{\tau}} \cdot d_{\phi_{\sigma}, \pm 1}\right)\left(s_{0}\right)= \pm \varepsilon\left(\wedge\left(\phi_{1}\right) \otimes \phi_{3}\right) \cdot \omega_{\pi_{1}}(-1) .
$$

Hence by the GP conjecture (Conjecture 5.2), we see that:

- $\operatorname{Hom}_{\mathrm{O}\left(V_{3}\right)}\left(\sigma_{i}^{+} \otimes \tau^{ \pm}, \mathbb{C}\right) \neq 0$ for some $i=1, \ldots, r$ if and only if $\pm \varepsilon\left(\wedge\left(\phi_{1}\right) \otimes\right.$ $\left.\phi_{3}\right) \cdot \omega_{\pi_{1}}(-1)=+1$

This implies Theorem 5.5 for tempered $\pi_{1}$ and $\pi_{3}$ such that $\omega_{\pi_{1}}^{2}=\omega_{\pi_{3}}=\mathbf{1}$.

In fact, there is an analogous theorem ([P4, Theorem 6]) for the quaternion division algebra case (in which case the product of the two root numbers is -1 ). If one knew $\iota_{1}\left({\sigma_{i}^{\prime}}^{ \pm}\right)$explicitly (as we have done for $\sigma_{i}^{ \pm}$by using Desideratum 3.12 (3)), one would show that this theorem follows from Conjecture 5.2 . Conversely, by using Prasad's theorem [P4, Theorem 6] and Conjecture 5.2, we may conclude that

$$
\iota_{1}\left({\sigma_{i}^{\prime}}^{ \pm}\right)\left(s_{0}\right)=\mp 1 .
$$

5.4. Proof of Conjecture $\mathbf{5 . 2}$. In this subsection, we prove that Prasad's conjecture (Conjecture 4.2) implies the Gross-Prasad conjecture (Conjecture 5.2). The second main theorem is as follows:

Theorem 5.6. Assume

- LLC for $\mathrm{O}\left(V_{m}\right) \times \mathrm{O}\left(V_{m+1}\right)$ (Desideratum 3.9 and Desideratum 3.1 for $\left.\mathrm{SO}\left(V_{\text {odd }}\right)\right)$;

- Hypothesis 3.13, which implies Prasad's conjecture (Conjecture 4.2) by Theorem 4.4 .

Then the Gross-Prasad conjecture (Conjecture 5.2) holds.

First, we consider the case when $m=2 n$ is even. We need the following lemma for this case:

Lemma 5.7. Let $\sigma_{0} \in \operatorname{Irr}\left(\mathrm{SO}\left(V_{2 n}^{\delta}\right)\right)$ and $\tau_{0} \in \operatorname{Irr}\left(\mathrm{SO}\left(V_{2 n+1}^{\delta}\right)\right)$. For $b \in\{ \pm 1\}$, we denote by $\tau^{b}$ the extension of $\tau_{0}$ to $\mathrm{O}\left(V_{2 n+1}^{\delta}\right)$ such that $\tau^{b}\left(-\mathbf{1}_{V_{2 n+1}^{\delta}}\right)=b \cdot \mathrm{id}$. Assume that

$$
\operatorname{Hom}_{\mathrm{SO}\left(V_{2 n}^{\delta}\right)}\left(\sigma_{0} \otimes \tau_{0}, \mathbb{C}\right) \neq 0
$$


(1) There exists a unique irreducible constituent $\sigma^{b}$ of $\operatorname{Ind}_{\mathrm{SO}\left(V_{2 n}^{\delta}\right)}^{\mathrm{O}\left(V_{2 n}^{\delta}\right)}\left(\sigma_{0}\right)$ such that

$$
\operatorname{Hom}_{\mathrm{O}\left(V_{2 n}^{\delta}\right)}\left(\sigma^{b} \otimes \tau^{b}, \mathbb{C}\right) \neq 0
$$

(2) If $\sigma_{0}^{\epsilon} \cong \sigma_{0}$, then the correspondence

$$
\{ \pm 1\} \ni b \mapsto \sigma^{b} \in\left\{\text { irreducible constituents of } \operatorname{Ind}_{\mathrm{SO}\left(V_{2 n}^{\delta}\right)}^{\mathrm{O}\left(V_{2 n}^{\delta}\right)}\left(\sigma_{0}\right)\right\}
$$

is bijective.

Proof. By the Frobenius reciprocity, we have

$$
\begin{aligned}
0 & \neq \operatorname{Hom}_{\mathrm{SO}\left(V_{2 n}^{\delta}\right)}\left(\sigma_{0} \otimes \tau_{0}, \mathbb{C}\right) \cong \operatorname{Hom}_{\mathrm{SO}\left(V_{2 n}^{\delta}\right)}\left(\tau_{0}, \sigma_{0}^{\vee}\right) \\
& \cong \operatorname{Hom}_{\mathrm{SO}\left(V_{2 n}^{\delta}\right)}\left(\tau^{b} \mid \mathrm{SO}\left(V_{2 n}^{\delta}\right), \sigma_{0}^{\vee}\right) \cong \operatorname{Hom}_{\mathrm{O}\left(V_{2 n}^{\delta}\right)}\left(\tau^{b} \mid \mathrm{O}\left(V_{2 n}^{\delta}\right), \operatorname{Ind}_{\mathrm{SO}\left(V_{2 n}^{\delta}\right)}^{\mathrm{O}\left(V_{2 n}^{\delta}\right)}\left(\sigma_{0}^{\vee}\right)\right) \\
& \cong \operatorname{Hom}_{\mathrm{O}\left(V_{2 n}^{\delta}\right)}\left(\operatorname{Ind}_{\mathrm{SO}\left(V_{2 n}^{\delta}\right)}^{\mathrm{O}\left(V_{2 n}^{\delta}\right)}\left(\sigma_{0}\right) \otimes \tau^{b}, \mathbb{C}\right)
\end{aligned}
$$

for any $b \in\{ \pm 1\}$. Hence if $\sigma_{0}^{\epsilon} ¥ \sigma_{0}$, then $\operatorname{Ind}_{\mathrm{SO}\left(V_{2 n}^{\delta}\right)}^{\mathrm{O}\left(V_{2 n}^{\delta}\right)}\left(\sigma_{0}\right)$ is irreducible, so that the first assertion is trivial.

Next, suppose that $\sigma_{0}^{\epsilon} \cong \sigma_{0}$. Then $\operatorname{Ind}_{\mathrm{SO}\left(V_{2 n}^{\delta}\right)}^{\mathrm{O}\left(V_{0}^{\delta}\right)}\left(\sigma_{0}\right) \cong \sigma_{1} \oplus \sigma_{2}$, where $\sigma_{1}, \sigma_{2} \in$ $\operatorname{Irr}\left(\mathrm{O}\left(V_{2 n}^{\delta}\right)\right)$ satisfy $\sigma_{1} \not \sigma_{2}$ and $\sigma_{i} \mid \mathrm{SO}\left(V_{2 n}^{\delta}\right)=\sigma_{0}$ for $i \in\{1,2\}$. Let $\epsilon \in \mathrm{O}\left(V_{2 n}^{\delta}\right)$ be as in 2.1. It satisfies that $\operatorname{det}(\epsilon)=-1$ and $\epsilon^{2}=\mathbf{1}_{V_{2 n}}$. Note that $\sigma_{2}(\epsilon)=-\sigma_{1}(\epsilon)$. Fix a non-zero homomorphism $f \in \operatorname{Hom}_{\mathrm{SO}\left(V_{2 n}^{\delta}\right)}\left(\sigma_{0} \otimes \tau_{0}, \mathbb{C}\right)$, and put

$$
f_{i}=f \circ\left(\sigma_{i}(\epsilon) \otimes \tau^{b}(\epsilon)\right) .
$$

Then $f_{i} \in \operatorname{Hom}_{\mathrm{SO}\left(V_{2 n}^{\delta}\right)}\left(\sigma_{0} \otimes \tau_{0}, \mathbb{C}\right)$, and we have $\left(f_{i}\right)_{i}=f$ and $\left(f_{1}\right)_{2}=-f$. Since $\operatorname{dim} \operatorname{Hom}_{\mathrm{SO}\left(V_{2 n}^{\delta}\right)}\left(\sigma_{0} \otimes \tau_{0}, \mathbb{C}\right)=1$ by [W4], there exists $c_{i} \in\{ \pm 1\}$ such that $f_{i}=c_{i} \cdot f$ and $c_{1} \neq c_{2}$. If $c_{i}=+1$, then $f \in \operatorname{Hom}_{\mathrm{O}\left(V_{2 n}^{\delta}\right)}\left(\sigma_{i} \otimes \tau^{b}, \mathbb{C}\right)$. In this case, we have $\sigma^{b}=\sigma_{i}$. Also, if we replace $b$ with $-b$, then $c_{i}$ must be replaced by $-c_{i}$. Hence the second assertion holds.

Proof of Theorem 5.6. First, we consider the case when $m=2 n$ is even. Let $\phi \in$ $\Phi_{\text {temp }}\left(\mathrm{O}\left(V_{2 n}\right)\right)$ and $\left(\phi^{\prime}, b\right) \in \Phi_{\text {temp }}\left(\mathrm{O}\left(V_{2 n+1}\right)\right)$. Theorem 5.1 and Lemma 5.7 imply that there exists a unique $(\sigma, \tau) \in \Pi_{\phi} \times \Pi_{\phi^{\prime}, b}$ such that $\sigma \otimes \tau$ is a representation of $\mathrm{O}\left(V_{2 n}^{\delta}\right) \times \mathrm{O}\left(V_{2 n+1}^{\delta}\right)$ for some $\delta \in\{ \pm 1\}$, and

$$
\operatorname{Hom}_{\Delta \mathrm{O}\left(V_{2 n}^{\delta}\right)}(\sigma \otimes \tau, \mathbb{C}) \neq 0 .
$$

Moreover, we have $\iota(\tau)=\chi_{\phi}$.

We show that $\iota_{c}(\sigma)=\chi_{\phi^{\prime}} \cdot d_{\phi, b}$. If $\phi \notin \Phi^{\epsilon}\left(\mathrm{O}\left(V_{2 n}\right)\right)$, then $A_{\phi}=A_{\phi}^{+}$and so $d_{\phi, b}=1$. Hence the desired equation follows from Theorem 5.1

Now we assume that $\phi \in \Phi_{\text {temp }}^{\epsilon}\left(\mathrm{O}\left(V_{2 n}\right)\right)$. Then $\sigma \neq \sigma \otimes$ det. Since $d_{\phi,-1}$ is the non-trivial character of $A_{\phi}$ which is trivial on $A_{\phi}^{+}$, by Lemma 5.7 (2), it suffices to show that if

$$
b=\varepsilon\left(\frac{1}{2}, \tau, \psi\right) \cdot \delta
$$

and $(\sigma, \tau) \in \Pi_{\phi} \times \Pi_{\phi^{\prime}, b}$ satisfies that $\operatorname{Hom}_{\Delta \mathrm{O}\left(V_{2 n}^{\delta}\right)}(\sigma \otimes \tau, \mathbb{C}) \neq 0$, then $\iota_{c}(\sigma)=$ $\chi_{\phi^{\prime}} \cdot d_{\phi, b}$. Here, $\varepsilon(s, \tau, \psi)$ is the standard $\varepsilon$-factor defined by the doubling method (see [LR, $\S 10])$. Note that

$$
\delta=\iota(\tau)\left(z_{\phi^{\prime}}\right)=\chi_{\phi}\left(z_{\phi^{\prime}}\right)=\varepsilon\left(\phi \otimes \phi^{\prime}\right) \cdot \chi_{V_{2 n}}(-1)^{n}
$$

by Theorem 5.1 . 
To obtain the desired formula, we shall use the theta correspondence as in 4.1 Let $W_{2 n}$ be the symplectic space of dimension $2 n$. We consider the theta correspondence for $\left(\mathrm{O}\left(V_{2 n}^{\delta}\right), \operatorname{Sp}\left(W_{2 n}\right)\right)$ and $\left(\mathrm{O}\left(V_{2 n+1}^{\delta}\right), \operatorname{Mp}\left(W_{2 n}\right)\right)$. Here, $\operatorname{Mp}\left(W_{2 n}\right)$ is the metaplectic group associated to $W_{2 n}$, i.e., the unique topological double cover of $\operatorname{Sp}\left(W_{2 n}\right)$. Since $\omega_{\tau}(-1)=b=\varepsilon(1 / 2, \tau, \psi) \delta$, by [GI1, Theorem 11.1], we have

$$
\Theta_{W_{2 n}, V_{2 n+1}^{\delta}, \psi}(\tau) \neq 0,
$$

so that $\rho:=\theta_{W_{2 n}, V_{2 n+1}^{\delta}, \psi}(\tau)$ is an irreducible genuine representation of $\operatorname{Mp}\left(W_{2 n}\right)$. Let $L$ be the orthogonal complement of $V_{2 n}^{\delta}$ in $V_{2 n+1}^{\delta}$. Note that $\operatorname{disc}(L)=-c$. Considering the following seesaw

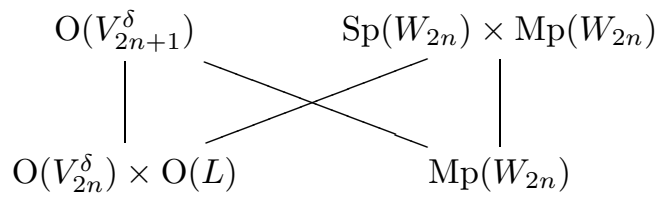

we see that

$$
\operatorname{Hom}_{\mathrm{Mp}\left(W_{2 n}\right)}\left(\Theta_{W_{2 n}, V_{2 n}^{\delta}, \psi}(\sigma) \otimes \omega_{\psi_{-c}}, \rho\right) \neq 0 .
$$

In particular, $\pi:=\Theta_{W_{2 n}, V_{2 n}^{\delta}, \psi}(\sigma)$ is non-zero. Since $\sigma$ is tempered, by [GI1, Proposition C.4], $\pi$ is irreducible, so that $\pi=\theta_{W_{2 n}, V_{2 n}^{\delta}, \psi}(\sigma)$. By Prasad's conjecture (Conjecture 4.2), we have $\pi \in \Pi_{\phi_{\pi}}$ with $\phi_{\pi}=(\phi \oplus \mathbf{1}) \otimes \chi_{V_{2 n}}$ and

$$
\iota_{c}(\pi) \mid A_{\phi}=\iota_{c}(\sigma) \text {. }
$$

Here, we regard $A_{\phi}$ as a subgroup of $A_{\phi_{\pi}}$ via the canonical injection $A_{\phi} \hookrightarrow A_{\phi_{\pi}}$. On the other hand, by the Gross-Prasad conjecture for the symplectic-metaplectic case, which has been established by At1, Theorem 1.3] (using Theorems 5.1, 4.7 and results of [GS]) and [GGP, Proposition 18.1], we have

$$
\iota_{c}(\pi)\left(a^{\prime}\right)=\varepsilon\left(\phi_{\pi}^{a^{\prime}} \chi_{c} \otimes \phi_{\rho \vee}\right) \cdot \varepsilon\left(\phi_{\pi} \chi_{c} \otimes \phi_{\rho^{\vee}}\right)^{\operatorname{det}\left(a^{\prime}\right)} \cdot \operatorname{det}\left(\phi_{\pi}^{a^{\prime}}\right)(-1)^{n}
$$

for $a^{\prime} \in A_{\phi_{\pi}}$. Here, $\phi_{\rho^{\vee}}$ is the $L$-parameter for $\rho^{\vee}$. It is given by $\phi_{\rho^{\vee}}=\phi^{\prime} \otimes$ $\chi_{-1} \chi_{V_{2 n+1}}$ (see [At1, §3.6]). Recall that $\iota_{c}(\pi)$ is a priori a character of $A_{\phi_{\pi}}^{+}$, but by using the isomorphism $A_{\phi_{\pi}}^{+} \cong A_{\phi_{\pi}} /\left\langle z_{\phi_{\pi}}\right\rangle$, we regard $\iota_{c}(\pi)$ as a character of $A_{\phi_{\pi}}$ which is trivial at $z_{\phi_{\pi}}$. If $a \in A_{\phi} \subset A_{\phi_{\pi}}$, then we have $\phi_{\pi}^{a}=\phi^{a} \otimes \chi_{V_{2 n}}$, so that

$$
\operatorname{det}\left(\phi_{\pi}^{a}\right)(-1)=\operatorname{det}\left(\phi^{a}\right)(-1) \cdot \chi_{V_{2 n}}(-1)^{\operatorname{dim}\left(\phi^{a}\right)} \text {. }
$$

Since $\operatorname{det}(a)=\operatorname{dim}\left(\phi^{a}\right)(\bmod 2)$ and $\chi_{V_{2 n}} \chi_{V_{2 n+1}} \chi_{c} \chi_{-1}=\mathbf{1}$, we have

$$
\varepsilon\left(\phi_{\pi}^{a} \chi_{c} \otimes \phi_{\rho^{\vee}}\right)=\varepsilon\left(\phi^{a} \otimes \phi^{\prime}\right)
$$

and

$$
\varepsilon\left(\phi_{\pi} \chi_{c} \otimes \phi_{\rho^{\vee}}\right)^{\operatorname{det}(a)}=\left(\varepsilon\left(\phi \otimes \phi^{\prime}\right) \cdot \varepsilon\left(\phi^{\prime}\right)\right)^{\operatorname{dim}\left(\phi^{a}\right)} .
$$

Since $\chi_{\phi^{\prime}}(a)=\varepsilon\left(\phi^{a} \otimes \phi^{\prime}\right) \cdot \operatorname{det}\left(\phi^{a}\right)(-1)^{n}$ and $\varepsilon\left(\phi^{\prime}\right) \cdot \varepsilon\left(\phi \otimes \phi^{\prime}\right) \cdot \chi_{V_{2 n}}(-1)^{n}=\varepsilon\left(\phi^{\prime}\right) \cdot \delta=b$, we have

$$
\iota_{c}(\sigma)(a)=\iota_{c}(\pi)(a)=\chi_{\phi^{\prime}}(a) \cdot b^{\operatorname{dim}\left(\phi^{a}\right)}
$$

for $a \in A_{\phi}$. This completes the proof of Theorem 5.6 when $m=2 n$.

Next, we consider the case when $m=2 n-1$ is odd. The proof is similar to that of [GGP, Theorem 19.1]. Let $\phi \in \Phi_{\text {temp }}\left(\mathrm{O}\left(V_{2 n}\right)\right)$ and $\left(\phi^{\prime}, b\right) \in \Phi_{\text {temp }}\left(\mathrm{O}\left(V_{2 n-1}\right)\right)$. 
Consider $V_{2 n-1}^{\delta} \subset V_{2 n}^{\delta}$. Let $L^{\delta}=F e_{0}$ be the orthogonal complement of $V_{2 n-1}^{\delta}$ in $V_{2 n}^{\delta}$ and $e_{0} \in L^{\delta}$ such that $\left\langle e_{0}, e_{0}\right\rangle_{V_{2 n}^{\delta}}=2 c$. Set

$$
V_{2 n+1}^{\delta}=V_{2 n}^{\delta} \oplus\left(-L^{\delta}\right)=V_{2 n}^{\delta} \oplus F f_{0},
$$

where $\left\langle f_{0}, f_{0}\right\rangle_{V_{2 n+1}^{\delta}}=-2 c$. Put $v_{0}=e_{0}+f_{0}$ and $X^{\delta}=F v_{0}$. Then we have

$$
V_{2 n+1}^{\delta}=X^{\delta} \oplus V_{2 n-1}^{\delta} \oplus\left(X^{\delta}\right)^{*}
$$

Let $P=M_{P} U_{P}$ be the parabolic subgroup of $\mathrm{O}\left(V_{2 n+1}^{\delta}\right)$ stabilizing the line $X^{\delta}$, where $M_{P} \cong \mathrm{GL}\left(X^{\delta}\right) \times \mathrm{O}\left(V_{2 n-1}^{\delta}\right)$ is the Levi subgroup of $P$ stabilizing $\left(X^{\delta}\right)^{*}$. Choose a unitary character $\chi$ of $F^{\times} \cong \mathrm{GL}\left(X^{\delta}\right)$ which satisfies the condition of GGP, Theorem 15,1], and such that the induced representation

$$
\operatorname{Ind}_{P\left(X^{\delta}\right)}^{\mathrm{O}\left(V_{2 n+1}^{\delta}\right)}(\chi \otimes \tau)
$$

is irreducible for any $\tau \in \Pi_{\phi^{\prime}, b}$. Note that in GGP], they consider the unnormalized induction, but in this paper, we consider the normalized induction. Then by GGP, Theorem 15,1], we have

$$
\operatorname{Hom}_{\mathrm{O}\left(V_{2 n}^{\delta}\right)}\left(\operatorname{Ind}_{P\left(X^{\delta}\right)}^{\mathrm{O}\left(V^{\delta}\right)}(\chi \otimes \tau) \otimes \sigma, \mathbb{C}\right) \cong \operatorname{Hom}_{\mathrm{O}\left(V_{2 n-1}^{\delta}\right)}(\tau \otimes \sigma, \mathbb{C}) .
$$

Put $\phi^{\prime \prime}=\chi \oplus \phi^{\prime} \oplus \chi^{-1}$. Then

$$
\Pi_{\phi^{\prime \prime}, \chi(-1) b}=\left\{\operatorname{Ind}_{P\left(X^{\delta}\right)}^{\mathrm{O}\left(V_{n+1}^{\delta}\right)}(\chi \otimes \tau) \mid \tau \in \Pi_{\phi^{\prime}, b}\right\}
$$

and

$$
\iota\left(\operatorname{Ind}_{P\left(X^{\delta}\right)}^{\mathrm{O}\left(V_{2 n+1}^{\delta}\right)}(\chi \otimes \tau)\right)=\iota(\tau)
$$

as a character of $A_{\phi^{\prime \prime}}=A_{\phi^{\prime}}$. Applying the even case above to $\phi \in \Phi_{\text {temp }}\left(\mathrm{O}\left(V_{2 n}\right)\right)$ and $\left(\phi^{\prime \prime}, \chi(-1) b\right) \in \Phi_{\text {temp }}\left(\mathrm{O}\left(V_{2 n+1}\right)\right)$, we see that there exists a unique pair $(\sigma, \tau) \in$ $\Pi_{\phi} \times \Pi_{\phi^{\prime}, b}$ such that $\sigma \otimes \tau$ is a representation of $\mathrm{O}\left(V_{2 n}^{\delta}\right) \times \mathrm{O}\left(V_{2 n-1}^{\delta}\right)$ for some $\delta \in\{ \pm 1\}$, and

$$
\operatorname{Hom}_{\Delta \mathrm{O}\left(V_{2 n-1}^{\delta}\right)}(\sigma \otimes \tau, \mathbb{C}) \neq 0
$$

Moreover, we have

$$
\begin{aligned}
\iota_{c}(\sigma)(a) & =\chi_{\phi^{\prime \prime}}(a) \cdot(\chi(-1) b)^{\operatorname{dim}\left(\phi^{a}\right)}, \\
\iota(\tau) & =\iota\left(\operatorname{Ind}_{P\left(X^{\delta}\right)}^{\mathrm{O}\left(V_{2 n+1}^{\delta}\right)}(\chi \otimes \tau)\right)=\chi_{\phi} .
\end{aligned}
$$

By the definition, we have

$$
\begin{aligned}
\frac{\chi_{\phi^{\prime \prime}}(a)}{\chi_{\phi^{\prime}}(a)} & =\varepsilon\left(\phi^{a} \otimes\left(\chi \oplus \chi^{-1}\right)\right) \cdot \operatorname{det}\left(\phi^{a}\right)(-1) \\
& =\operatorname{det}\left(\phi^{a} \otimes \chi\right)(-1) \cdot \operatorname{det}\left(\phi^{a}\right)(-1)=\chi(-1)^{\operatorname{dim}\left(\phi^{a}\right)} .
\end{aligned}
$$

Hence we have

$$
\iota_{c}(\sigma)(a)=\chi_{\phi^{\prime}}(a) \cdot b^{\operatorname{dim}\left(\phi^{a}\right)},
$$

as desired. This completes the proof of Theorem 5.6 when $m=2 n-1$. 
By Theorem 5.6, we have established the Gross-Prasad conjecture (Conjecture 5.2) under Prasad's conjecture (Conjecture 4.2). As in GGP, one may consider the general codimension case. It is a restriction problem for $G=\mathrm{O}\left(V_{m}\right) \times \mathrm{O}\left(V_{n}\right) \supset H=$ $N \rtimes \mathrm{O}\left(V_{n}\right)$, where $V_{m}$ is an orthogonal space of dimension $m, V_{n}$ is a non-degenerate subspace of $V_{m}$ of dimension $n$ such that

- $m-n$ is odd;

- the orthogonal complement of $V_{n}$ in $V_{m}$ is split,

and $N$ is the unipotent radical of a parabolic subgroup of $\mathrm{SO}\left(V_{m}\right)$. By the same method of the proof as GGP, Theorem 19.1], the general codimension case (for tempered $L$-parameters) would follow from the codimension 1 case (Conjecture 5.2 .

\section{Arthur's multiplicity formula for $\mathrm{SO}\left(V_{2 n}\right)$}

The final main theorem is Arthur's multiplicity formula, which describes a spectral decomposition of the discrete automorphic spectrum for $\mathrm{O}\left(V_{2 n}\right)$. In this section, we recall the local and global $A$-parameters, and Arthur's multiplicity formula for $\mathrm{SO}\left(V_{2 n}\right)$. Then we will establish an analogous formula for $\mathrm{O}\left(V_{2 n}\right)$ in the next section.

6.1. Notation and measures. Let $\mathbb{F}$ be a number field, $\mathbb{A}$ be the ring of adeles of $\mathbb{F}$. We denote by $\mathbb{A}_{\text {fin }}=\prod_{v<\infty}^{\prime} \mathbb{F}_{v}$ and $\mathbb{F}_{\infty}=\prod_{v \mid \infty} \mathbb{F}_{v}$ the ring of finite adeles and infinite adeles, respectively. As in the previous sections, we write $V_{2 n}$ for the orthogonal space associated to $(d, c)$ for some $c, d \in \mathbb{F}^{\times}$. Let $\mathrm{O}\left(V_{2 n}\right)\left(\operatorname{resp} . \mathrm{SO}\left(V_{2 n}\right)\right)$ be a quasi-split orthogonal (resp. special orthogonal) group over $\mathbb{F}$. We denote by $\chi_{V}=\otimes_{v} \chi_{V, v}: \mathbb{A}^{\times} / \mathbb{F}^{\times} \rightarrow \mathbb{C}^{\times}$the discriminant character.

For each $v$, we fix a maximal compact subgroup $K_{v}$ of $\mathrm{O}\left(V_{2 n}\right)\left(\mathbb{F}_{v}\right)$ such that $K_{v}$ is special if $v$ is non-archimedean. Moreover, if $\mathrm{O}\left(V_{2 n}\right)\left(\mathbb{F}_{v}\right)$ is unramified, we choose $K_{v}$ as in $\$ 2.3$ which is hyperspecial. Also, we take $\epsilon_{v} \in K_{v}$ such that $\operatorname{det}\left(\epsilon_{v}\right)=-1, \epsilon_{v}^{2}=\mathbf{1}_{V_{2 n}}$ and that $\epsilon=\left(\epsilon_{v}\right)_{v} \in \mathrm{O}\left(V_{2 n}\right)(\mathbb{A})$ is in $\mathrm{O}\left(V_{2 n}\right)(\mathbb{F})$. Put $K_{0, v}=K_{v} \cap \operatorname{SO}\left(V_{2 n}\right)\left(\mathbb{F}_{v}\right)$. Note that $\epsilon_{v}^{-1} K_{0, v} \epsilon_{v}=K_{0, v}$.

Let $\mu_{2}=\{ \pm 1\}$ be the group of order 2 . We regard $\mu_{2}$ as an algebraic group over $\mathbb{F}$. There exists an exact sequence of algebraic groups over $\mathbb{F}$ :

$$
1 \longrightarrow \mathrm{SO}\left(V_{2 n}\right) \longrightarrow \mathrm{O}\left(V_{2 n}\right) \stackrel{\text { det }}{\longrightarrow} \mu_{2} \longrightarrow 1 \text {. }
$$

For $t=\left(t_{v}\right)_{v} \in \mu_{2}(\mathbb{A})$, we define $\epsilon_{t}=\left(\epsilon_{t, v}\right)_{v} \in \mathrm{O}\left(V_{2 n}\right)(\mathbb{A})$ by

$$
\epsilon_{t, v}= \begin{cases}\mathbf{1}_{V_{2 n}} & \text { if } t_{v}=1 \\ \epsilon_{v} & \text { if } t_{v}=-1\end{cases}
$$

We take the Haar measures $d g_{v}, d h_{v}$, and $d t_{v}$ on $\mathrm{O}\left(V_{2 n}\right)\left(\mathbb{F}_{v}\right), \mathrm{SO}\left(V_{2 n}\right)\left(\mathbb{F}_{v}\right)$ and $\mu_{2}\left(\mathbb{F}_{v}\right)$, respectively, so that

$$
\operatorname{vol}\left(K_{v}, d g_{v}\right)=\operatorname{vol}\left(K_{0, v}, d h_{v}\right)=\operatorname{vol}\left(\mu_{2}\left(\mathbb{F}_{v}\right), d t_{v}\right)=1 .
$$

Then they induce the Haar measures $d g, d h$ and $d t$ on $\mathrm{O}\left(V_{2 n}\right)(\mathbb{A}), \mathrm{SO}\left(V_{2 n}\right)(\mathbb{A})$ and $\mu_{2}(\mathbb{A})$, respectively, satisfying that

$$
\int_{\mathrm{O}\left(V_{2 n}\right)(\mathbb{F}) \backslash \mathrm{O}\left(V_{2 n}\right)(\mathbb{A})} f(g) d g=\int_{\mu_{2}(\mathbb{F}) \backslash \mu_{2}(\mathbb{A})}\left(\int_{\mathrm{SO}\left(V_{2 n}\right)(\mathbb{F}) \backslash \mathrm{SO}\left(V_{2 n}\right)(\mathbb{A})} f\left(h \epsilon_{t}\right) d h\right) d t
$$

for any smooth function $f$ on $\mathrm{O}\left(V_{2 n}\right)(\mathbb{F}) \backslash \mathrm{O}\left(V_{2 n}\right)(\mathbb{A})$. 
6.2. Local $A$-parameters. In this subsection, we fix a place $v$ of $\mathbb{F}$, and introduce local $A$-parameters for $\mathrm{O}\left(V_{2 n}\right)\left(\mathbb{F}_{v}\right)$ and $\mathrm{SO}\left(V_{2 n}\right)\left(\mathbb{F}_{v}\right)$.

Let $W_{\mathbb{F}_{v}}$ be the Weil group and $W D_{\mathbb{F}_{v}}$ be the Weil-Deligne group of $\mathbb{F}_{v}$, i.e.,

$$
W D_{\mathbb{F}_{v}}= \begin{cases}W_{\mathbb{F}_{v}} \times \mathrm{SL}_{2}(\mathbb{C}) & \text { if } v \text { is non-archimedean, } \\ W_{\mathbb{F}_{v}} & \text { if } v \text { is archimedean. }\end{cases}
$$

A local $A$-parameter for $\mathrm{SO}\left(V_{2 n}\right)\left(\mathbb{F}_{v}\right)$ is a homomorphism

$$
\underline{\psi}: W D_{\mathbb{F}_{v}} \times \mathrm{SL}_{2}(\mathbb{C}) \rightarrow{ }^{L}\left(\mathrm{SO}\left(V_{2 n}\right)\right)=\mathrm{SO}(2 n, \mathbb{C}) \rtimes W_{\mathbb{F}_{v}}
$$

which commutes the projections $W D_{\mathbb{F}_{v}} \times \mathrm{SL}_{2}(\mathbb{C}) \rightarrow W_{\mathbb{F}_{v}}$ and ${ }^{L}\left(\mathrm{SO}\left(V_{2 n}\right)\right) \rightarrow W_{\mathbb{F}_{v}}$, and such that $\psi\left(W_{\mathbb{F}_{v}}\right)$ projects onto a relatively compact subset of $\mathrm{SO}(2 n, \mathbb{C})$. We put

$$
\begin{aligned}
& \Psi\left(\mathrm{SO}\left(V_{2 n}\right)\left(\mathbb{F}_{v}\right)\right) \\
& \quad=\left\{\mathrm{SO}(2 n, \mathbb{C}) \text {-conjugacy classes of local } A \text {-parameters of } \mathrm{SO}\left(V_{2 n}\right)\left(\mathbb{F}_{v}\right)\right\} .
\end{aligned}
$$

In 33.2 , we have defined a map ${ }^{L}\left(\mathrm{SO}\left(V_{2 n}\right)\right) \rightarrow \mathrm{O}(2 n, \mathbb{C})$. By composing with this map, $\underline{\psi} \in \Psi\left(\mathrm{SO}\left(V_{2 n}\right)\left(\mathbb{F}_{v}\right)\right)$ gives a representation

$$
\psi: W D_{\mathbb{F}_{v}} \times \mathrm{SL}_{2}(\mathbb{C}) \rightarrow \mathrm{O}(2 n, \mathbb{C}) .
$$

We may regard $\psi$ as an orthogonal representation of $W D_{\mathbb{F}_{v}} \times \mathrm{SL}_{2}(\mathbb{C})$. The map $\underline{\psi} \mapsto \psi$ gives an identification

$$
\begin{aligned}
& \Psi\left(\mathrm{SO}\left(V_{2 n}\right)\left(\mathbb{F}_{v}\right)\right) \\
& =\left\{\psi: W D_{\mathbb{F}_{v}} \times \mathrm{SL}_{2}(\mathbb{C}) \rightarrow \mathrm{O}(2 n, \mathbb{C}) \mid \operatorname{det}(\psi)=\chi_{V, v}\right\} /(\mathrm{SO}(2 n, \mathbb{C}) \text {-conjugacy }) .
\end{aligned}
$$

Namely, we regard $\Psi\left(\mathrm{SO}\left(V_{2 n}\right)\left(\mathbb{F}_{v}\right)\right)$ as the set of $\mathrm{SO}(M)$-conjugacy classes of orthogonal representations $(\psi, M)$ of $W D_{\mathbb{F}_{v}} \times \mathrm{SL}_{2}(\mathbb{C})$ with $\operatorname{dim}(M)=2 n$ and $\operatorname{det}(\psi)=$ $\chi_{V, v}$. We say that $\psi \in \Psi\left(\mathrm{SO}\left(V_{2 n}\right)\left(\mathbb{F}_{v}\right)\right)$ is tempered if $\psi \mid \mathrm{SL}_{2}(\mathbb{C})=\mathbf{1}$, i.e., $\psi$ is a tempered representation of $W D_{\mathbb{F}_{v}}$. We denote by $\Psi_{\text {temp }}\left(\mathrm{SO}\left(V_{2 n}\right)\left(\mathbb{F}_{v}\right)\right)$ the subset of $\Psi\left(\mathrm{SO}\left(V_{2 n}\right)\left(\mathbb{F}_{v}\right)\right)$ consisting of the classes of tempered representations. Also we put $\Psi\left(\operatorname{SO}\left(V_{2 n}\right)\left(\mathbb{F}_{v}\right)\right) / \sim_{\epsilon}$ to be the set of equivalence classes of orthogonal representations $(\psi, M)$ of $W D_{\mathbb{F}_{v}} \times \mathrm{SL}_{2}(\mathbb{C})$ with $\operatorname{dim}(M)=2 n$ and $\operatorname{det}(\psi)=\chi_{V, v}$. Then there exists a canonical surjection

$$
\Psi\left(\mathrm{SO}\left(V_{2 n}\right)\left(\mathbb{F}_{v}\right)\right) \rightarrow \Psi\left(\mathrm{SO}\left(V_{2 n}\right)\left(\mathbb{F}_{v}\right)\right) / \sim_{\epsilon}
$$

such that the order of each fiber is one or two. Denote by $\Psi_{\text {temp }}\left(\operatorname{SO}\left(V_{2 n}\right)\left(\mathbb{F}_{v}\right)\right) / \sim_{\epsilon}$ the image of $\Psi_{\text {temp }}\left(\mathrm{SO}\left(V_{2 n}\right)\left(\mathbb{F}_{v}\right)\right)$.

On the other hand, we put

$$
\begin{aligned}
& \Psi\left(\mathrm{O}\left(V_{2 n}\right)\left(\mathbb{F}_{v}\right)\right) \\
& \quad=\left\{\mathrm{O}(2 n, \mathbb{C}) \text {-conjugacy classes of local } A \text {-parameters of } \mathrm{SO}\left(V_{2 n}\right)\left(\mathbb{F}_{v}\right)\right\} .
\end{aligned}
$$

We call an element in $\Psi\left(\mathrm{O}\left(V_{2 n}\right)\left(\mathbb{F}_{v}\right)\right)$ an $A$-parameter of $\mathrm{O}\left(V_{2 n}\right)\left(\mathbb{F}_{v}\right)$. Then we have a canonical identification $\Psi\left(\mathrm{O}\left(V_{2 n}\right)\left(\mathbb{F}_{v}\right)\right)=\Psi\left(\mathrm{SO}\left(V_{2 n}\right)\left(\mathbb{F}_{v}\right)\right) / \sim_{\epsilon}$. Under this identification, we put $\Psi_{\text {temp }}\left(\mathrm{O}\left(V_{2 n}\right)\left(\mathbb{F}_{v}\right)\right)=\Psi_{\text {temp }}\left(\mathrm{SO}\left(V_{2 n}\right)\left(\mathbb{F}_{v}\right)\right) / \sim_{\epsilon}$.

Let $\psi \in \Psi\left(\mathrm{O}\left(V_{2 n}\right)\left(\mathbb{F}_{v}\right)\right)=\Psi\left(\mathrm{SO}\left(V_{2 n}\right)\left(\mathbb{F}_{v}\right)\right) / \sim_{\epsilon}$ be a local $A$-parameter. We put

$$
\mathcal{S}_{\psi}=\pi_{0}\left(\operatorname{Cent}\left(\operatorname{Im}(\psi), \mathrm{O}_{2 n}(\mathbb{C})\right) /\left\{ \pm \mathbf{1}_{2 n}\right\}\right)
$$

and

$$
\mathcal{S}_{\psi}^{+}=\pi_{0}\left(\operatorname{Cent}\left(\operatorname{Im}(\psi), \mathrm{SO}_{2 n}(\mathbb{C})\right) /\left\{ \pm \mathbf{1}_{2 n}\right\}\right)
$$


6.3. Local $A$-packets. We denote by $\operatorname{Irr}_{\text {unit }}\left(\mathrm{O}\left(V_{2 n}\right)\right)\left(\mathbb{F}_{v}\right)$ the set of equivalence classes of irreducible unitary representations of $\mathrm{O}\left(V_{2 n}\right)\left(\mathbb{F}_{v}\right)$. By Theorems 2.2.1 and 2.2.4 in $\left[\mathrm{Ar}\right.$, there exists a finite set $\Pi_{\psi}$ with maps

$$
\Pi_{\psi} \rightarrow \operatorname{Irr}_{\text {unit }}\left(\mathrm{O}\left(V_{2 n}\right)\left(\mathbb{F}_{v}\right)\right)
$$

and

$$
\iota_{c}: \Pi_{\psi} \rightarrow \widehat{\mathcal{S}_{\psi}},
$$

which satisfy certain character identities. Using the multiplicity function

$$
m_{1}: \operatorname{Irr}_{\text {unit }}\left(\mathrm{O}\left(V_{2 n}\right)\left(\mathbb{F}_{v}\right)\right) \rightarrow \mathbb{Z}_{\geq 0}
$$

which gives the order of the fibers in $\Pi_{\psi}$, we may regard $\Pi_{\psi}$ as a multiset on $\operatorname{Irr}_{\text {unit }}\left(\mathrm{O}\left(V_{2 n}\right)\left(\mathbb{F}_{v}\right)\right)$. We call $\Pi_{\psi}$ the local $A$-packets for $\mathrm{O}\left(V_{2 n}\right)\left(\mathbb{F}_{v}\right)$ associated to $\psi$. Note that $m_{1}(\sigma)=m_{1}(\sigma \otimes$ det $)$ by $\mathrm{Ar}$, Theorem 2.2.4]. If $\psi$ is tempered, $\Pi_{\psi}$ coincides with the $L$-packet described in $\$ 3.6$. In particular, if $\psi$ is tempered, then $\Pi_{\psi}$ is multiplicity-free.

We denote by $\Pi_{\psi}^{0}$ the image of $\Pi_{\psi}$ under the canonical map

$$
\operatorname{Irr}_{\text {unit }}\left(\mathrm{O}\left(V_{2 n}\right)\left(\mathbb{F}_{v}\right)\right) \rightarrow \operatorname{Irr}_{\text {unit }}\left(\mathrm{O}\left(V_{2 n}\right)\left(\mathbb{F}_{v}\right)\right) / \sim_{\text {det }} \rightarrow \operatorname{Irr}_{\text {unit }}\left(\mathrm{SO}\left(V_{2 n}\right)\left(\mathbb{F}_{v}\right)\right) / \sim_{\epsilon} .
$$

Namely, $\Pi_{\psi}^{0}$ is a multiset on $\operatorname{Irr}_{\text {unit }}\left(\operatorname{SO}\left(V_{2 n}\right)\left(\mathbb{F}_{v}\right)\right) / \sim_{\epsilon}$ with the multiplicity function

$$
m_{0}: \operatorname{Irr}_{\text {unit }}\left(\operatorname{SO}\left(V_{2 n}\right)\left(\mathbb{F}_{v}\right)\right) / \sim_{\epsilon} \rightarrow \mathbb{Z}_{\geq 0}
$$

such that

$$
m_{0}([\sigma])=m_{1}(\sigma)=m_{1}(\sigma \otimes \operatorname{det})
$$

where $[\sigma] \in \operatorname{Irr}_{\text {unit }}\left(\mathrm{SO}\left(V_{2 n}\right)\left(\mathbb{F}_{v}\right)\right) / \sim_{\epsilon}$ is the image of $\sigma \in \operatorname{Irr}_{\text {unit }}\left(\mathrm{O}\left(V_{2 n}\right)\left(\mathbb{F}_{v}\right)\right)$. We call $\Pi_{\psi}^{0}$ the local $A$-packets for $\mathrm{SO}\left(V_{2 n}\right)\left(\mathbb{F}_{v}\right)$ associated to $\psi$. Moreover there exists a map

$$
\iota_{c}: \Pi_{\psi}^{0} \rightarrow \widehat{\mathcal{S}_{\psi}^{+}}
$$

which satisfies certain character identities and such that the diagram

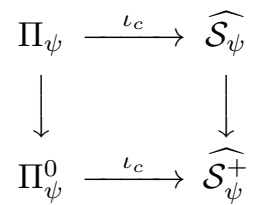

is commutative.

Recall that the local $A$-packets $\Pi_{\psi}$ and $\Pi_{\psi}^{0}$ are multisets. However, it is expected that the $A$-packets are sets.

Conjecture 6.1. Let $\psi \in \Psi\left(\mathrm{O}\left(V_{2 n}\right)\left(\mathbb{F}_{v}\right)\right)=\Psi\left(\mathrm{SO}\left(V_{2 n}\right)\left(\mathbb{F}_{v}\right)\right) / \sim_{\epsilon}$ be a local $A$ parameter, and $\Pi_{\psi}$ and $\Pi_{\psi}^{0}$ be the local A-packets. Then for $\sigma \in \operatorname{Irr}_{\text {unit }}\left(\mathrm{O}\left(V_{2 n}\right)\right)$ and $[\sigma] \in \operatorname{Irr}_{\text {unit }}\left(\mathrm{SO}\left(V_{2 n}\right)\left(\mathbb{F}_{v}\right)\right) / \sim_{\epsilon}$, we have

$$
m_{1}(\sigma) \leq 1 \text { and } m_{0}([\sigma]) \leq 1 .
$$

In other words, $\Pi_{\psi}$ and $\Pi_{\psi}^{0}$ are subsets of

$$
\operatorname{Irr}_{\text {unit }}\left(\mathrm{O}\left(V_{2 n}\right)\right) \text { and } \operatorname{Irr}_{\text {unit }}\left(\mathrm{SO}\left(V_{2 n}\right)\left(\mathbb{F}_{v}\right)\right) / \sim_{\epsilon},
$$

respectively.

Proposition 6.2. Conjecture 6.1 for $\psi$ holds for the following cases:

- when $\psi=\phi$ is a tempered A-parameter;

- when $\mathbb{F}_{v}$ is non-archimedean;

- when $\mathbb{F}_{v}=\mathbb{C}$ is the complex field. 
Proof. For a tempered $A$-parameter $\psi=\phi$, the local $A$-packet $\Pi_{\psi}$ coincides with the local $L$-packet $\Pi_{\phi}$, which are (multiplicity-free) sets. When $\mathbb{F}_{v}$ is non-archimedean, Conjecture 6.1 is proven by Mœglin [M2. See also Xu's paper [X, Theorem 8.12]. When $\mathbb{F}_{v}=\mathbb{C}$, it is proven by Møglin-Renard [MR1].

Remark 6.3. By Proposition 6.2, only when $\mathbb{F}_{v}=\mathbb{R}$ is the real number field and $\psi$ is non-tempered, Conjecture 6.1 for $\psi$ is not verified. However, it is known by AMR that a part of local $A$-packets (in the archimedean case) coincides with Adams-Johnson $A$-packets, which are (multiplicity-free) sets.

Let $\psi \in \Psi\left(\mathrm{O}\left(V_{2 n}\right)\left(\mathbb{F}_{v}\right)\right)=\Psi\left(\mathrm{SO}\left(V_{2 n}\right)\left(\mathbb{F}_{v}\right)\right) / \sim_{\epsilon}$. Then $\psi$ gives a local $L$ parameter $\phi_{\psi}$ defined by

$$
\phi_{\psi}(w)=\psi\left(w,\left(\begin{array}{cc}
|w|_{\mathbb{F}_{v}}^{\frac{1}{2}} & 0 \\
0 & |w|_{\mathbb{F}_{v}}^{-\frac{1}{2}}
\end{array}\right)\right)
$$

for $w \in W D_{\mathbb{F}_{v}}$. Here, $|w|_{\mathbb{F}_{v}}$ is the extension to $W D_{\mathbb{F}_{v}}$ of the absolute value on $W_{\mathbb{F}_{v}}$, which is trivial on $\mathrm{SL}_{2}(\mathbb{C})$. We put

$$
\mathcal{S}_{\phi_{\psi}}=\pi_{0}\left(\operatorname{Cent}\left(\operatorname{Im}\left(\phi_{\psi}\right), \mathrm{O}_{2 n}(\mathbb{C})\right) /\left\{ \pm \mathbf{1}_{2 n}\right\}\right)
$$

and

$$
\mathcal{S}_{\phi_{\psi}}^{+}=\pi_{0}\left(\operatorname{Cent}\left(\operatorname{Im}\left(\phi_{\psi}\right), \mathrm{SO}_{2 n}(\mathbb{C})\right) /\left\{ \pm \mathbf{1}_{2 n}\right\}\right) .
$$

Then we have $L$-packets

$$
\Pi_{\phi_{\psi}} \subset \operatorname{Irr}_{\text {unit }}\left(\mathrm{O}\left(V_{2 n}\right)\left(\mathbb{F}_{v}\right)\right) \text { and } \Pi_{\phi_{\psi}}^{0} \subset \operatorname{Irr}_{\text {unit }}\left(\mathrm{SO}\left(V_{2 n}\right)\left(\mathbb{F}_{v}\right)\right) / \sim_{\epsilon}
$$

and bijections

$$
\iota_{c}: \Pi_{\phi_{\psi}} \rightarrow \widehat{\mathcal{S}_{\phi_{\psi}}} \text { and } \iota_{c}: \Pi_{\phi_{\psi}}^{0} \rightarrow \widehat{\mathcal{S}_{\phi_{\psi}}^{+}}
$$

Moreover we have canonical surjections

$$
\mathcal{S}_{\psi} \rightarrow \mathcal{S}_{\phi_{\psi}} \quad \text { and } \quad \mathcal{S}_{\psi}^{+} \rightarrow \mathcal{S}_{\phi_{\psi}}^{+} .
$$

Proposition 7.4.1 in $\underline{\mathrm{Ar}}$ s says that $\Pi_{\phi_{\psi}}^{0}$ is contained in $\Pi_{\psi}^{0}$ and the diagram

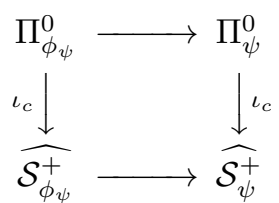

is commutative. We prove an analogue of this statement.

Proposition 6.4. Assume Conjecture 6.1. Let $\psi \in \Psi\left(\mathrm{O}\left(V_{2 n}\right)\left(\mathbb{F}_{v}\right)\right)$. We denote by $\phi_{\psi}$ the L-parameter given by $\psi$. Let $\Pi_{\psi}$ be the local A-packet of $\psi$, which is a multiset of $\operatorname{Irr}_{\text {unit }}\left(\mathrm{O}\left(V_{2 n}\right)\left(\mathbb{F}_{v}\right)\right)$, and $\Pi_{\phi_{\psi}}$ be the local L-packet of $\phi_{\psi}$, which is a subset of $\operatorname{Irr}_{\text {unit }}\left(\mathrm{O}\left(V_{2 n}\right)\left(\mathbb{F}_{v}\right)\right)$. Then $\Pi_{\phi_{\psi}}$ is contained in $\Pi_{\psi}$ and the diagram

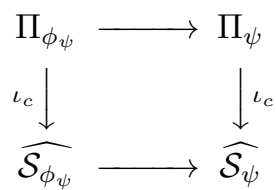

is commutative. 
Proof. The first assertion follows from the result for $\Pi_{\phi_{\psi}}^{0}$ and $\Pi_{\psi}^{0}$. We write $\iota_{c}^{L}=$ $\iota_{c}: \Pi_{\phi_{\psi}} \rightarrow \widehat{\mathcal{S}_{\phi_{\psi}}}$ for the left arrow in the diagram, and $\iota_{c}^{A}=\iota_{c}: \Pi_{\psi} \rightarrow \widehat{\mathcal{S}_{\psi}}$ for the right arrow in the diagram. Let $a \in \mathcal{S}_{\psi}$ and $s \in \mathrm{O}_{2 n}(\mathbb{C})$ be a (suitable) semi-simple representative of $a$. We denote by $\psi^{a}$ the $(-1)$-eigenspace of $s$ in $\psi$, which is a representation of $W D_{\mathbb{F}_{v}} \times \mathrm{SL}_{2}(\mathbb{C})$. For the last assertion, we may assume that $d=\operatorname{dim}\left(\psi^{a}\right)$ is odd. Put $\psi^{\prime}=\psi \oplus \psi^{a} \oplus\left(\psi^{a}\right)^{\vee}$. Then we have

$$
\phi_{\psi^{\prime}}=\phi_{\psi} \oplus \phi_{\psi}^{a} \oplus\left(\phi_{\psi}^{a}\right)^{\vee} \text {. }
$$

Take $\sigma \in \Pi_{\phi_{\psi}}$. Let $\tau$ be an irreducible representation of $\mathrm{GL}_{d}\left(\mathbb{F}_{v}\right)$ corresponding to $\phi_{\psi}^{a}$. We denote the normalized intertwining operator of $\operatorname{Ind}_{P}^{\mathrm{O}\left(V_{2 n+2 d}\right)\left(\mathbb{F}_{v}\right)}(\tau \otimes \sigma)$ by $R_{c}(w, \tau \otimes \sigma)$, where $P=M N$ is a suitable parabolic subgroup of $\mathrm{O}\left(V_{2 n+2 d}\right)\left(\mathbb{F}_{v}\right)$ with the Levi factor $M \cong \mathrm{GL}_{d}\left(\mathbb{F}_{v}\right) \times \mathrm{O}\left(V_{2 n}\right)\left(\mathbb{F}_{v}\right)$, and $w$ is a suitable representative of an element in the relative Weyl group $W(M)$ of $M$. Let $\sigma^{\prime}$ be an irreducible constituent of $\operatorname{Ind}_{P}^{\mathrm{O}\left(V_{2 n+2 d}\right)\left(\mathbb{F}_{v}\right)}(\tau \otimes \sigma)$. Then $\sigma^{\prime} \in \Pi_{\phi_{\psi^{\prime}}} \subset \Pi_{\psi^{\prime}}$. Regarding $\sigma^{\prime}$ as an element in $\Pi_{\phi_{\psi^{\prime}}}$, by Theorems 2.2.4 and 2.4.4 in [Ar] together with Conjecture 6.1. $R_{c}(w, \sigma \otimes \tau)$ induces a scalar operator on $\sigma^{\prime}$ with eigenvalue $\iota_{c}^{L}\left(\sigma^{\prime}\right)(a)$. On the other hand, regarding $\sigma^{\prime}$ as an element in $\Pi_{\psi^{\prime}}$, by the same theorems and conjecture, $R_{c}(w, \sigma \otimes \tau)$ induces a scalar operator on $\sigma^{\prime}$ with eigenvalue $\iota_{c}^{A}\left(\sigma^{\prime}\right)(a)$. Hence

$$
\iota_{c}^{L}\left(\sigma^{\prime}\right)(a)=\iota_{c}^{A}\left(\sigma^{\prime}\right)(a) .
$$

Since $\iota_{c}^{L}\left(\sigma^{\prime}\right)(a)=\iota_{c}^{L}(\sigma)(a)$ and $\iota_{c}^{A}\left(\sigma^{\prime}\right)(a)=\iota_{c}^{A}(\sigma)(a)$, we have

$$
\iota_{c}^{L}(\sigma)(a)=\iota_{c}^{A}(\sigma)(a),
$$

as desired.

We remark on unramified representations. Suppose that $\mathrm{O}\left(V_{2 n}\right)\left(\mathbb{F}_{v}\right)$ is unramified, or equivalently, that $v$ is non-archimedean and $c, d \in \mathfrak{o}_{v}^{\times}$, where $\mathfrak{o}_{v}$ is the ring of integers of $\mathbb{F}_{v}$. We say that $\psi \in \Psi\left(\mathrm{O}\left(V_{2 n}\right)\left(\mathbb{F}_{v}\right)\right)$ is unramified if $\psi \mid W D_{\mathbb{F}_{v}}$ is trivial on $I_{\mathbb{F}_{v}} \times \mathrm{SL}_{2}(\mathbb{C})$, where $I_{\mathbb{F}_{v}}$ is the inertia subgroup of $W_{\mathbb{F}_{v}}$.

Corollary 6.5. If $\psi \in \Psi\left(\mathrm{O}\left(V_{2 n}\right)\left(\mathbb{F}_{v}\right)\right)$ is unramified, then $\Pi_{\psi}$ has a unique unramified representation $\sigma$. It satisfies $\iota_{c}(\sigma)=\mathbf{1}$.

Proof. The uniqueness follows from [M1, §4.4, Proposition] and Lemma 2.4, The unique unramified representation $\sigma \in \Pi_{\psi}$ belongs to the subset $\Pi_{\phi_{\psi}}$. By Proposition 6.4 and Desideratum 3.12 (4), we have $\iota_{c}(\sigma)=1$.

6.4. Hypothetical Langlands group and its substitute. We denote the set of irreducible unitary cuspidal automorphic representations of $\mathrm{GL}_{m}(\mathbb{A})$ by $\mathcal{A}_{\text {cusp }}\left(\mathrm{GL}_{m}\right)$. Let $\mathcal{L}_{\mathbb{F}}$ be the hypothetical Langlands group of $\mathbb{F}$. It is expected that there is a surjection $\mathcal{L}_{\mathbb{F}} \rightarrow W_{\mathbb{F}}$, where $W_{\mathbb{F}}$ is the Weil group of $\mathbb{F}$, and there exists a canonical bijection

$$
\mathcal{A}_{\text {cusp }}\left(\mathrm{GL}_{m}\right) \longleftrightarrow\left\{m \text {-dimensional irreducible unitary representations of } \mathcal{L}_{\mathbb{F}}\right\} \text {. }
$$

We want to use $\mathcal{L}_{\mathbb{F}}$ as a global analogue of Weil-Deligne group $W D_{\mathbb{F}_{v}}$. Namely, for a connected reductive group $G$, we want to define a global $A$-parameters of $G$ by a homomorphism

$$
\psi: \mathcal{L}_{\mathbb{F}} \times \mathrm{SL}_{2}(\mathbb{C}) \rightarrow{ }^{L} G=\widehat{G} \rtimes W_{\mathbb{F}}
$$

which commutes the projections $\mathcal{L}_{\mathbb{F}} \rightarrow W_{\mathbb{F}}$ and ${ }^{L} G \rightarrow W_{\mathbb{F}}$. 
In this paper, we do not assume the existence of $\mathcal{L}_{\mathbb{F}}$. So we have to modify the definition of global $A$-parameters. For the definition of global $A$-parameters, we use elements in $\mathcal{A}_{\text {cusp }}\left(\mathrm{GL}_{m}\right)$ instead of $m$-dimensional irreducible unitary representations of $\mathcal{L}_{\mathbb{F}}$. For this reason, we will define not $\Psi_{2}\left(\mathrm{SO}\left(V_{2 n}\right)\right)$ but only $\Psi_{2}\left(\mathrm{SO}\left(V_{2 n}\right)\right) / \sim_{\epsilon}$ as well as $\Psi_{2}\left(\mathrm{O}\left(V_{2 n}\right)\right)$ in the next subsection.

6.5. Global $A$-parameters and their localization. Let $V_{2 n}$ be the orthogonal space associated to $(d, c)$ for some $c, d \in \mathbb{F}^{\times}$. We denote by $\chi_{V}: \mathbb{A}^{\times} / \mathbb{F}^{\times} \rightarrow \mathbb{C}^{\times}$ the discriminant character of $V_{2 n}$. A discrete global $A$-parameter for $\mathrm{SO}\left(V_{2 n}\right)$ and $\mathrm{O}\left(V_{2 n}\right)$ is a symbol

$$
\Sigma=\Sigma_{1}\left[d_{1}\right] \boxplus \cdots \boxplus \Sigma_{l}\left[d_{l}\right]
$$

where

- $1 \leq l \leq 2 n$ is an integer;

- $\Sigma_{i} \in \mathcal{A}_{\text {cusp }}\left(\mathrm{GL}_{m_{i}}\right)$;

- $d_{i}$ is a positive integer such that $\sum_{i=1}^{l} m_{i} d_{i}=2 n$;

- if $d_{i}$ is odd, then $L\left(s, \Sigma_{i}, \operatorname{Sym}^{2}\right)$ has a pole at $s=1$;

- if $d_{i}$ is even, then $L\left(s, \Sigma_{i}, \wedge^{2}\right)$ has a pole at $s=1$;

- if we denote the central character of $\Sigma_{i}$ by $\omega_{i}$, then $\omega_{1}^{d_{1}} \cdots \omega_{l}^{d_{l}}=\chi_{V}$;

- if $i \neq j$ and $\Sigma_{i} \cong \Sigma_{j}$, then $d_{i} \neq d_{j}$.

Two global $A$-parameters $\Sigma=\Sigma_{1}\left[d_{1}\right] \boxplus \cdots \boxplus \Sigma_{l}\left[d_{l}\right]$ and $\Sigma^{\prime}=\Sigma_{1}^{\prime}\left[d_{1}^{\prime}\right] \boxplus \cdots \boxplus \Sigma_{l^{\prime}}^{\prime}\left[d_{l^{\prime}}^{\prime}\right]$ are said to be equivalent if $l=l^{\prime}$ and there exists a permutation $\sigma \in \mathfrak{S}_{l}$ such that $d_{i}^{\prime}=$ $d_{\sigma(i)}$ and $\Sigma_{i}^{\prime} \cong \Sigma_{\sigma(i)}$ for each $i$. We denote by $\Psi_{2}\left(\mathrm{O}\left(V_{2 n}\right)\right)=\Psi_{2}\left(\mathrm{SO}\left(V_{2 n}\right)\right) / \sim_{\epsilon}$ the set of equivalence classes of discrete global $A$-parameters for $\mathrm{SO}\left(V_{2 n}\right)$ and $\mathrm{O}\left(V_{2 n}\right)$. Let $\Psi_{2}^{\epsilon}\left(\mathrm{O}\left(V_{2 n}\right)\right)$ be the subset of $\Psi_{2}\left(\mathrm{O}\left(V_{2 n}\right)\right)$ consisting of $\Sigma=\boxplus_{i=1}^{l} \Sigma_{i}\left[d_{i}\right]$ as above such that $m_{i} d_{i}$ is odd for some $i$. Also, we put

$$
\Psi_{2, \text { temp }}\left(\mathrm{O}\left(V_{2 n}\right)\right)=\left\{\Sigma=\Sigma_{1}\left[d_{1}\right] \boxplus \cdots \boxplus \Sigma_{l}\left[d_{l}\right] \in \Psi_{2}\left(\mathrm{O}\left(V_{2 n}\right)\right) \mid d_{i}=1 \text { for any } i\right\},
$$

and $\Psi_{2, \text { temp }}^{\epsilon}\left(\mathrm{O}\left(V_{2 n}\right)\right)=\Psi_{2, \text { temp }}\left(\mathrm{O}\left(V_{2 n}\right)\right) \cap \Psi_{2}^{\epsilon}\left(\mathrm{O}\left(V_{2 n}\right)\right)$. We define $\Psi_{2}^{\epsilon}\left(\mathrm{SO}\left(V_{2 n}\right)\right) / \sim_{\epsilon}$ and $\Psi_{2, \text { temp }}\left(\mathrm{SO}\left(V_{2 n}\right)\right) / \sim_{\epsilon}$ similarly.

Let $\Sigma=\Sigma_{1}\left[d_{1}\right] \boxplus \cdots \boxplus \Sigma_{l}\left[d_{l}\right] \in \Psi_{2}\left(\mathrm{O}\left(V_{2 n}\right)\right)$. For each place $v$ of $\mathbb{F}$, we denote the $m_{i}$-dimensional representation of $W D_{\mathbb{F}_{v}}$ corresponding to $\Sigma_{i, v} \in \operatorname{Irr}\left(\mathrm{GL}_{m_{i}}\left(\mathbb{F}_{v}\right)\right)$ by $\phi_{i, v}$. Because of the lack of the generalized Ramanujan conjecture, $\phi_{i, v}$ is not necessarily a tempered representation. We define a representation $\Sigma_{v}: W D_{\mathbb{F}_{v}} \times$ $\mathrm{SL}_{2}(\mathbb{C}) \rightarrow \mathrm{GL}_{2 n}(\mathbb{C})$ by

$$
\Sigma_{v}=\left(\phi_{1, v} \otimes S_{d_{1}}\right) \oplus \cdots \oplus\left(\phi_{l, v} \otimes S_{d_{l}}\right),
$$

where $S_{d}$ is the unique irreducible algebraic representation of $\mathrm{SL}_{2}(\mathbb{C})$ of dimension $d$. We call $\Sigma_{v}$ the localization of $\Sigma$ at $v$. By [Ar, Proposition 1.4.2], the representation $\Sigma_{v}$ factors through $\mathrm{O}_{2 n}(\mathbb{C}) \hookrightarrow \mathrm{GL}_{2 n}(\mathbb{C})$. In particular, if $\Sigma \in \Psi_{2, \text { temp }}\left(\mathrm{O}\left(V_{2 n}\right)\right)$, then $\Sigma_{v} \in \Phi\left(\mathrm{O}\left(V_{2 n}\right)\left(\mathbb{F}_{v}\right)\right)$.

Let $\Sigma=\Sigma_{1}\left[d_{1}\right] \boxplus \cdots \boxplus \Sigma_{l}\left[d_{l}\right] \in \Psi_{2}\left(\mathrm{O}\left(V_{2 n}\right)\right)$ be a global $A$-parameter with $\Sigma_{i} \in$ $\mathcal{A}_{\text {cusp }}\left(\mathrm{GL}_{m_{i}}\right)$, and $\Sigma_{v}=\left(\phi_{1, v} \otimes S_{d_{1}}\right) \oplus \cdots \oplus\left(\phi_{l, v} \otimes S_{d_{l}}\right)$ be the localization at $v$. We also write $\Sigma_{i, v}=\phi_{i, v} \otimes S_{d_{i}}$. We put

$$
A_{\Sigma}=\bigoplus_{i=1}^{l}(\mathbb{Z} / 2 \mathbb{Z}) a_{\Sigma_{i}\left[d_{i}\right]} \cong(\mathbb{Z} / 2 \mathbb{Z})^{l} .
$$

Namely, $A_{\Sigma}$ is a free $(\mathbb{Z} / 2 \mathbb{Z})$-module of rank $l$ and $\left\{a_{\Sigma_{1}\left[d_{1}\right]}, \ldots, a_{\Sigma_{l}\left[d_{l}\right]}\right\}$ is a basis of $A_{\Sigma}$ with $a_{\Sigma_{i}\left[d_{i}\right]}$ associated to $\Sigma_{i}\left[d_{i}\right]$. We define $A_{\Sigma}^{+}$by the kernel of the map 
$A_{\Sigma} \ni a_{\Sigma_{i}\left[d_{i}\right]} \mapsto(-1)^{m_{i} d_{i}} \in\{ \pm 1\}$. Also, we put

$$
z_{\Sigma}=\sum_{i=1}^{l} a_{\Sigma_{i}\left[d_{i}\right]} \in A_{\Sigma}^{+} \subset A_{\Sigma} .
$$

We define the global component groups $\mathcal{S}_{\Sigma}$ and $\mathcal{S}_{\Sigma}^{+}$by

$$
\mathcal{S}_{\Sigma}=A_{\Sigma} /\left\langle z_{\phi}\right\rangle \text { and } \mathcal{S}_{\Sigma}^{+}=A_{\Sigma}^{+} /\left\langle z_{\phi}\right\rangle .
$$

On the other hand, as in 6.2 , we put

$$
\mathcal{S}_{\Sigma_{v}}=\pi_{0}\left(\operatorname{Cent}\left(\operatorname{Im}\left(\Sigma_{v}\right), \mathrm{O}_{2 n}(\mathbb{C})\right) /\left\{ \pm \mathbf{1}_{2 n}\right\}\right)
$$

and

$$
\mathcal{S}_{\Sigma_{v}}^{+}=\pi_{0}\left(\operatorname{Cent}\left(\operatorname{Im}\left(\Sigma_{v}\right), \mathrm{SO}_{2 n}(\mathbb{C})\right) /\left\{ \pm \mathbf{1}_{2 n}\right\}\right) .
$$

Then we have a map

$$
\mathcal{S}_{\Sigma} \rightarrow \mathcal{S}_{\Sigma_{v}}, a_{\Sigma_{i}\left[d_{i}\right]} \mapsto-\mathbf{1}_{\Sigma_{i, v}}
$$

for each place $v$, where $-\mathbf{1}_{\Sigma_{i, v}}$ is the image of the element in $\operatorname{Cent}\left(\operatorname{Im}\left(\Sigma_{v}\right), \mathrm{O}_{2 n}(\mathbb{C})\right)$ which acts on $\Sigma_{i, v}$ by $-\mathrm{id}$ and acts on $\Sigma_{j, v}$ trivially for any $j \neq i$. Hence we obtain the diagonal maps

$$
\Delta: \mathcal{S}_{\Sigma} \rightarrow \prod_{v} \mathcal{S}_{\Sigma_{v}} \quad \text { and } \quad \Delta: \mathcal{S}_{\Sigma}^{+} \rightarrow \prod_{v} \mathcal{S}_{\Sigma_{v}}^{+}
$$

Let $\Sigma \in \Psi_{2}\left(\mathrm{O}\left(V_{2 n}\right)\right)$ be a global $A$-parameter and $\psi=\Sigma_{v}$ be the localization at $v$. We emphasize that $\psi$ does not necessarily belong to $\Psi\left(\mathrm{O}\left(V_{2 n}\right)\left(\mathbb{F}_{v}\right)\right)$ defined in 6.2. We can decompose

$$
\psi=\left.\left.\left.\left.\psi_{1}|\cdot|\right|_{\mathbb{F}_{v}} ^{s_{1}} \oplus \cdots \oplus \psi_{r}|\cdot|\right|_{\mathbb{F}_{v}} ^{s_{r}} \oplus \psi_{0} \oplus \psi_{r}^{\vee}|\cdot|\right|_{\mathbb{F}_{v}} ^{-s_{r}} \oplus \cdots \oplus \psi_{1}^{\vee}|\cdot|\right|_{\mathbb{F}_{v}} ^{-s_{1}},
$$

where

- $\psi_{i}$ is an irreducible representation of $W D_{\mathbb{F}_{v}} \times \mathrm{SL}_{2}(\mathbb{C})$ of dimension $d_{i}$ such that $\psi_{i}\left(W_{\mathbb{F}_{v}}\right)$ is bounded;

- $\psi_{0} \in \Psi\left(\mathrm{O}\left(V_{2 n_{0}}\right)\left(\mathbb{F}_{v}\right)\right)$;

- $d_{1}+\cdots+d_{r}+n_{0}=n$ and $s_{1} \geq \cdots \geq s_{r}>0$.

We define a representation $\phi_{\psi_{i}}$ of $W D_{\mathbb{F}_{v}}$ by

$$
\phi_{\psi_{i}}(w)=\psi_{i}\left(w,\left(\begin{array}{cc}
|w|_{\mathbb{F}_{v}}^{\frac{1}{2}} & 0 \\
0 & |w|_{\mathbb{F}_{v}}^{-\frac{1}{2}}
\end{array}\right)\right),
$$

and we denote by $\tau_{\psi_{i}}$ the irreducible representation of $\mathrm{GL}_{d_{i}}\left(\mathbb{F}_{v}\right)$ corresponding to $\phi_{\psi_{i}}$. Let $\Pi_{\psi_{0}}$ be the local $A$-packet associated to $\psi_{0}$, which is a multiset of $\operatorname{Irr}_{\text {unit }}\left(\mathrm{O}\left(V_{2 n_{0}}\right)\left(\mathbb{F}_{v}\right)\right)$. For $\sigma_{0} \in \Pi_{\psi_{0}}$, we put

$$
I\left(\sigma_{0}\right)=\operatorname{Ind}_{P\left(\mathbb{F}_{v}\right)}^{\mathrm{O}\left(V_{2 n}\right)\left(\mathbb{F}_{v}\right)}\left(\left.\left.\tau_{\psi_{1}}|\cdot|\right|_{\mathbb{F}_{v}} ^{s_{1}} \otimes \cdots \otimes \tau_{\psi_{r}}|\cdot|\right|_{\mathbb{F}_{v}} ^{s_{r}} \otimes \sigma_{0}\right)
$$

where $P$ is a parabolic subgroup of $\mathrm{O}\left(V_{2 n}\right)$ with Levi subgroup $M_{P}=\mathrm{GL}_{d_{1}} \times \cdots \times$ $\mathrm{GL}_{d_{r}} \times \mathrm{O}\left(V_{2 n_{0}}\right)$. Note that $I\left(\sigma_{0}\right)$ depends on not only $\sigma_{0}$ but also $\psi$.

To establish the global main result (Theorem 7.1 below), we need the following conjecture (see $\mathrm{Ar}$, Conjecture 8.3.1]).

Conjecture 6.6. Let $\Sigma \in \Psi_{2}\left(\mathrm{O}\left(V_{2 n}\right)\right)$ be a global A-parameter. We decompose $\Sigma_{v}$ as above, so that $\psi_{0} \in \Psi\left(\mathrm{O}\left(V_{2 n_{0}}\right)\left(\mathbb{F}_{v}\right)\right)$. Then the induced representation $I\left(\sigma_{0}\right)$ is irreducible for $\sigma_{0} \in \Pi_{\psi_{0}}$. Moreover, if $I\left(\sigma_{0}\right) \cong I\left(\sigma_{0}^{\prime}\right)$ for $\sigma_{0}, \sigma_{0}^{\prime} \in \Pi_{\psi_{0}}$, then $\sigma_{0} \cong \sigma_{0}^{\prime}$. 
Proposition 6.7. Conjecture 6.6 holds for the following cases:

- when $\Sigma \in \Psi_{2, \text { temp }}\left(\mathrm{O}\left(V_{2 n}\right)\right)$;

- when $\mathbb{F}_{v}$ is non-archimedean;

- when $\mathbb{F}_{v}=\mathbb{C}$ is the complex field.

Proof. The second case is [M3, Proposition 5.1]. The third case is proven in [MR1, Théorème 6.6, Remarque 6.7]. So we shall prove the first case.

The first case follows from a result of Heiermann $[\mathrm{H}$. Let $\Sigma$ be an element in $\Psi_{2 \text {,temp }}\left(\mathrm{O}\left(V_{2 n}\right)\right), \phi=\Sigma_{v}$ be the localization of $\Sigma$ at $v$, and $\phi_{0}=\psi_{0}$ be as above. Note that $\phi \in \Phi\left(\mathrm{O}\left(V_{2 n}\right)\left(\mathbb{F}_{v}\right)\right)$. Then the $L$-packet $\Pi_{\phi}$ is equal to the set of the Langlands quotients of the standard module $I\left(\sigma_{0}\right)$, where $\sigma_{0}$ runs over $\Pi_{\phi_{0}}$. Heiermann's result $[\mathrm{H}]$ (together with the Clifford theory) asserts that if $\phi \in \Phi_{\text {gen }}\left(\mathrm{O}\left(V_{2 n}\right)\left(\mathbb{F}_{v}\right)\right)$, then $I\left(\sigma_{0}\right)$ is irreducible for any $\sigma_{0} \in \Pi_{\phi_{0}}$. Namely, to show the irreducibility of $I\left(\sigma_{0}\right)$, it suffices to prove that $L(s, \phi, \mathrm{Ad})$ is regular at $s=1$.

We decompose

$$
\phi=\left.\left.\left.\left.\phi_{1}|\cdot|\right|_{\mathbb{F}_{v}} ^{s_{1}} \oplus \cdots \oplus \phi_{r}|\cdot|\right|_{\mathbb{F}_{v}} ^{s_{r}} \oplus \phi_{0} \oplus \phi_{r}^{\vee}|\cdot|\right|_{\mathbb{F}_{v}} ^{-s_{r}} \oplus \cdots \oplus \phi_{1}^{\vee}|\cdot|\right|_{\mathbb{F}_{v}} ^{-s_{1}},
$$

where

- $\phi_{i}$ is an irreducible tempered representation of $W D_{\mathbb{F}_{v}}$ of dimension $d_{i}$;

- $\phi_{0} \in \Phi_{\text {temp }}\left(\mathrm{O}\left(V_{2 n_{0}}\right)\left(\mathbb{F}_{v}\right)\right)$;

- $d_{1}+\cdots+d_{r}+n_{0}=n$ and $s_{1} \geq \cdots \geq s_{r}>0$.

Then $L(s, \phi, \mathrm{Ad})$ is equal to

$$
\begin{gathered}
L\left(s, \phi_{0}, \mathrm{Ad}\right)\left(\prod_{i=1}^{r} L\left(s, \phi_{i}, \operatorname{Ad}_{\mathrm{GL}}\right) L\left(s+s_{i}, \phi_{0} \otimes \phi_{i}\right) L\left(s-s_{i}, \phi_{0} \otimes \phi_{i}^{\vee}\right)\right. \\
\left.\times L\left(s+2 s_{i}, \phi_{i}, \wedge^{2}\right) L\left(s-2 s_{i}, \phi_{i}^{\vee}, \wedge^{2}\right)\right) \\
\times\left(\prod_{1 \leq i<j \leq r} L\left(s+s_{i}+s_{j}, \phi_{i} \otimes \phi_{j}\right) L\left(s+s_{i}-s_{j}, \phi_{i} \otimes \phi_{j}^{\vee}\right)\right. \\
\left.\times L\left(s-s_{i}+s_{j}, \phi_{i}^{\vee} \otimes \phi_{j}\right) L\left(s-s_{i}-s_{j}, \phi_{i}^{\vee} \otimes \phi_{j}^{\vee}\right)\right),
\end{gathered}
$$

where $\mathrm{Ad}_{\mathrm{GL}}$ is the adjoint representation of $\mathrm{GL}_{m}(\mathbb{C})$ on $\operatorname{Lie}\left(\mathrm{GL}_{m}(\mathbb{C})\right)$ for a suitable $m$.

Since $\phi$ corresponds to an irreducible representation of $\mathrm{GL}_{2 n}\left(\mathbb{F}_{v}\right)$, which is a local constituent of $\Sigma=\boxplus_{i=1}^{l} \Sigma_{i}$, we have $\left|s_{i}\right|<1 / 2$ for any $i$. See e.g., [JS, (2.5) Corollary] and [RS, Appendix]. Note that for tempered representations, all $L$ functions appearing in the above equation are regular for $\operatorname{Re}(s)>0$. Hence we conclude that $L(s, \phi, \mathrm{Ad})$ is regular at $s=1$.

Since $I\left(\sigma_{0}\right)$ is a standard module, the last assertion of Conjecture 6.6 follows from the Langlands classification.

Go back to the general situation. Let $\Sigma \in \Psi_{2}\left(\mathrm{O}\left(V_{2 n}\right)\right)$ be a global $A$-parameter, and $\psi=\Sigma_{v}, \psi_{0}$ and $I\left(\sigma_{0}\right)$ for $\sigma_{0} \in \Pi_{\psi_{0}}$ be as above. We define the local $A$-packet 
$\Pi_{\psi}$ associated to $\psi=\Sigma_{v}$, which is a multiset on $\operatorname{Irr}\left(\mathrm{O}\left(V_{2 n}\right)\left(\mathbb{F}_{v}\right)\right)$, by

$$
\Pi_{\psi}=\bigsqcup_{\sigma_{0} \in \Pi_{\psi_{0}}}\left\{\sigma \mid \sigma \text { is an irreducible constituent of } I\left(\sigma_{0}\right)\right\} .
$$

Namely, $\Pi_{\psi}$ is the disjoint union of the multisets of the Jordan-Hölder series of $I\left(\sigma_{0}\right)$. Similarly, we can define the local $A$-packet $\Pi_{\psi}^{0}$ associated to $\psi=\Sigma_{v}$, which is a multiset on $\operatorname{Irr}\left(\operatorname{SO}\left(V_{2 n}\right)\left(\mathbb{F}_{v}\right)\right) / \sim_{\epsilon}$. Since $\mathcal{S}_{\psi}=\mathcal{S}_{\psi_{0}}$ and $\mathcal{S}_{\psi}^{+}=\mathcal{S}_{\psi_{0}}^{+}$, we can define maps

$$
\iota_{c}: \Pi_{\psi} \rightarrow \widehat{\mathcal{S}_{\psi}} \quad \text { and } \quad \iota_{c}: \Pi_{\psi}^{0} \rightarrow \widehat{\mathcal{S}_{\psi}^{+}}
$$

by

$$
\iota_{c}(\sigma):=\iota_{c}\left(\sigma_{0}\right) \quad \text { and } \quad \iota_{c}([\sigma]):=\iota_{c}\left(\left[\sigma_{0}\right]\right)
$$

if $\sigma$ is an irreducible constituent of $I\left(\sigma_{0}\right)$.

6.6. Global $A$-packets. Let $\mathcal{H}\left(\mathrm{O}\left(V_{2 n}\right)\right)=\otimes_{v}^{\prime} \mathcal{H}\left(\mathrm{O}\left(V_{2 n}\right)\left(\mathbb{F}_{v}\right)\right)\left(\operatorname{resp} . \mathcal{H}\left(\mathrm{SO}\left(V_{2 n}\right)\right)=\right.$ $\left.\otimes_{v}^{\prime} \mathcal{H}\left(\mathrm{SO}\left(V_{2 n}\right)\left(\mathbb{F}_{v}\right)\right)\right)$ be the global Hecke algebra on $\mathrm{O}\left(V_{2 n}\right)(\mathbb{A})\left(\operatorname{resp} . \mathrm{SO}\left(V_{2 n}\right)(\mathbb{A})\right)$ with respect to the maximal compact subgroup $K=\prod_{v} K_{v}\left(\operatorname{resp} . K_{0}=\prod_{v} K_{0, v}\right)$ fixed in 6.1 Namely, $\mathcal{H}\left(\mathrm{O}\left(V_{2 n}\right)\left(\mathbb{F}_{v}\right)\right)$ (resp. $\mathcal{H}\left(\mathrm{SO}\left(V_{2 n}\right)\left(\mathbb{F}_{v}\right)\right)$ ) is the $\mathbb{C}$-algebra of smooth, left and right $K_{v}$-finite (resp. $K_{0, v}$-finite) functions of compact support on $\mathrm{O}\left(V_{2 n}\right)\left(\mathbb{F}_{v}\right)$ (resp. $\left.\mathrm{SO}\left(V_{2 n}\right)\left(\mathbb{F}_{v}\right)\right)$. We denote by $\mathcal{H}^{\epsilon}\left(\mathrm{SO}\left(V_{2 n}\right)\left(\mathbb{F}_{v}\right)\right)$ the subspace of functions in $\mathcal{H}\left(\mathrm{SO}\left(V_{2 n}\right)\left(\mathbb{F}_{v}\right)\right)$ which are invariant under $\epsilon_{v}$. We put $\mathcal{H}^{\epsilon}\left(\mathrm{SO}\left(V_{2 n}\right)\right)=\otimes_{v}^{\prime} \mathcal{H}^{\epsilon}\left(\mathrm{SO}\left(V_{2 n}\right)\left(\mathbb{F}_{v}\right)\right)$. We say that two admissible representations of $\mathrm{SO}\left(V_{2 n}\right)(\mathbb{A})$ of the forms

$$
\sigma_{0}=\otimes_{v}^{\prime} \sigma_{0, v} \quad \text { and } \quad \sigma_{0}^{\prime}=\otimes_{v}^{\prime} \sigma_{0, v}^{\prime}
$$

are $\epsilon$-equivalent if $\sigma_{0, v} \sim_{\epsilon_{v}} \sigma_{0, v}^{\prime}$ for each $v$. The $\epsilon$-equivalence class of $\sigma_{0}$ is denoted by $\left[\sigma_{0}\right]=\otimes_{v}^{\prime}\left[\sigma_{0, v}\right]$. For $f \in \mathcal{H}^{\epsilon}\left(\operatorname{SO}\left(V_{2 n}\right)\right)$ and any $\sigma_{0}$ as above, the operator

$$
\sigma_{0}(f): \sigma_{0} \rightarrow \sigma_{0}, \sigma_{0}(f) v=\int_{\mathrm{SO}\left(V_{2 n}\right)(\mathbb{A})} f(h) \sigma_{0}(h) v d h
$$

depends only on the class $\left[\sigma_{0}\right]$.

Let $\Sigma \in \Psi_{2}\left(\mathrm{O}\left(V_{2 n}\right)\right)$ be a global $A$-parameter. We attach a global $A$-packet

$$
\Pi_{\Sigma}=\left\{\sigma=\otimes_{v}^{\prime} \sigma_{v} \mid \sigma_{v} \in \Pi_{\Sigma_{v}}, \iota_{c}\left(\sigma_{v}\right)=1 \text { for almost all } v\right\}
$$

of equivalence classes of irreducible representations of $\mathrm{O}\left(V_{2 n}\right)(\mathbb{A})$, and a global $A$ packet

$$
\Pi_{\Sigma}^{0}=\left\{\left[\sigma_{0}\right]=\otimes_{v}^{\prime}\left[\sigma_{0, v}\right] \mid\left[\sigma_{0, v}\right] \in \Pi_{\Sigma_{v}}^{0}, \iota_{c}\left(\left[\sigma_{0, v}\right]\right)=\mathbf{1} \text { for almost all } v\right\}
$$

of $\epsilon$-equivalence classes of irreducible representations of $\mathrm{SO}\left(V_{2 n}\right)(\mathbb{A})$. Note that an element $\sigma \in \Pi_{\Sigma}$ and a representative $\sigma_{0}$ of $\left[\sigma_{0}\right] \in \Pi_{\Sigma}^{0}$ are not necessarily unitary. For $\sigma \in \Pi_{\Sigma}$ and $\left[\sigma_{0}\right] \in \Pi_{\Sigma}^{0}$, the operators $\sigma(f)$ and $\sigma_{0}\left(f_{0}\right)$ are well-defined for $f \in \mathcal{H}\left(\mathrm{O}\left(V_{2 n}\right)\right)$ and $f_{0} \in \mathcal{H}^{\epsilon}\left(\mathrm{SO}\left(V_{2 n}\right)\right)$, respectively.

For $\sigma=\otimes_{v}^{\prime} \sigma_{v} \in \Pi_{\Sigma}$ and $\left[\sigma_{0}\right]=\otimes_{v}^{\prime}\left[\sigma_{0, v}\right] \in \Pi_{\Sigma}^{0}$, we define characters $\iota_{c}(\sigma)$ of $\prod_{v} \mathcal{S}_{\Sigma_{v}}$ and $\iota_{c}\left(\left[\sigma_{0}\right]\right)$ of $\prod_{v} \mathcal{S}_{\Sigma_{v}}^{+}$by

$$
\iota_{c}(\sigma)=\prod_{v} \iota_{c}\left(\sigma_{v}\right) \quad \text { and } \quad \iota_{c}\left(\left[\sigma_{0}\right]\right)=\prod_{v} \iota_{c}\left(\left[\sigma_{0, v}\right]\right)
$$

respectively. 
6.7. Arthur's multiplicity formula for $\mathrm{SO}\left(V_{2 n}\right)$. We say that a function

$$
\varphi: \mathrm{O}\left(V_{2 n}\right)(\mathbb{A}) \rightarrow \mathbb{C}
$$

is an automorphic form on $\mathrm{O}\left(V_{2 n}\right)(\mathbb{A})$ if $\varphi$ satisfies the following conditions:

- $\varphi$ is left $\mathrm{O}\left(V_{2 n}\right)(\mathbb{F})$-invariant;

- $\varphi$ is smooth and of moderate growth;

- $\varphi$ is right $K$-finite, where $K=\prod_{v} K_{v}$ is the maximal compact subgroup of $\mathrm{O}\left(V_{2 n}\right)(\mathbb{A})$ fixed in $\$ 6.1$.

- $\varphi$ is $\mathfrak{z}$-finite, where $\mathfrak{z}$ is the center of the universal enveloping algebra of $\mathrm{Lie}\left(\mathrm{O}\left(V_{2 n}\right)\left(\mathbb{F}_{\infty}\right)\right) \otimes_{\mathbb{R}} \mathbb{C}$.

We define automorphic forms on $\mathrm{SO}\left(V_{2 n}\right)(\mathbb{A})$ similarly. For more precision, see [BJ, §4.2]. Let $\mathcal{A}\left(\mathrm{O}\left(V_{2 n}\right)\right)$ be the space of automorphic forms on $\mathrm{O}\left(V_{2 n}\right)(\mathbb{A})$. We denote by $\mathcal{A}_{2}\left(\mathrm{O}\left(V_{2 n}\right)\right)$ the subspace of $\mathcal{A}\left(\mathrm{O}\left(V_{2 n}\right)\right)$ consisting of square-integrable automorphic forms on $\mathrm{O}\left(V_{2 n}\right)(\mathbb{A})$. Similarly, we define $\mathcal{A}\left(\mathrm{SO}\left(V_{2 n}\right)\right)$ and $\mathcal{A}_{2}\left(\mathrm{SO}\left(V_{2 n}\right)\right)$. We call $\mathcal{A}_{2}\left(\mathrm{O}\left(V_{2 n}\right)\right)$ (resp. $\mathcal{A}_{2}\left(\mathrm{SO}\left(V_{2 n}\right)\right)$ ) the automorphic discrete spectrum of $\mathrm{O}\left(V_{2 n}\right)$ (resp. $\left.\mathrm{SO}\left(V_{2 n}\right)\right)$.

The Hecke algebras $\mathcal{H}\left(\mathrm{O}\left(V_{2 n}\right)\right)$ and $\mathcal{H}\left(\mathrm{SO}\left(V_{2 n}\right)\right)$ act on $\mathcal{A}\left(\mathrm{O}\left(V_{2 n}\right)\right)$ and $\mathcal{A}\left(\mathrm{SO}\left(V_{2 n}\right)\right)$, respectively, by

$$
\begin{aligned}
(f \cdot \varphi)(g) & =\int_{\mathrm{O}\left(V_{2 n}\right)(\mathbb{A})} \varphi(g x) f(x) d x, \\
\left(f_{0} \cdot \varphi_{0}\right)\left(g_{0}\right) & =\int_{\mathrm{SO}\left(V_{2 n}\right)(\mathbb{A})} \varphi_{0}\left(g_{0} x_{0}\right) f_{0}\left(x_{0}\right) d x_{0},
\end{aligned}
$$

for $f \in \mathcal{H}\left(\mathrm{O}\left(V_{2 n}\right)\right), \varphi \in \mathcal{A}\left(\mathrm{O}\left(V_{2 n}\right)\right), f_{0} \in \mathcal{H}\left(\mathrm{SO}\left(V_{2 n}\right)\right)$ and $\varphi_{0} \in \mathcal{A}\left(\mathrm{SO}\left(V_{2 n}\right)\right)$. These actions preserve $\mathcal{A}_{2}\left(\mathrm{O}\left(V_{2 n}\right)\right)$ and $\mathcal{A}_{2}\left(\mathrm{SO}\left(V_{2 n}\right)\right)$, respectively.

Arthur's multiplicity formula for $\mathrm{SO}\left(V_{2 n}\right)$ is formulated as follows:

Theorem 6.8 (Arthur's multiplicity formula ( $\mathrm{Ar}$, Theorem 1.5.2])). Let $V_{2 n}$ be the orthogonal space over a number field $\mathbb{F}$ associated to $(d, c)$ for some $c, d \in \mathbb{F}^{\times}$. Then for each $\Sigma \in \Psi_{2}\left(\mathrm{SO}\left(V_{2 n}\right)\right) / \sim_{\epsilon}$, there exists a character

$$
\varepsilon_{\Sigma}: \mathcal{S}_{\Sigma} \rightarrow\{ \pm 1\}
$$

defined explicitly in terms of symplectic $\varepsilon$-factors such that

$$
\mathcal{A}_{2}\left(\mathrm{SO}\left(V_{2 n}\right)\right) \cong \bigoplus_{\Sigma \in \Psi_{2}\left(\operatorname{SO}\left(V_{2 n}\right)\right) / \sim_{\epsilon}} m_{\Sigma} \bigoplus_{\left[\sigma_{0}\right] \in \Pi_{\Sigma}^{0}\left(\varepsilon_{\Sigma}\right)}\left[\sigma_{0}\right]
$$

as $\mathcal{H}^{\epsilon}\left(\mathrm{SO}\left(V_{2 n}\right)\right)$-modules. Here for $\Sigma=\boxplus_{i=1}^{l} \Sigma_{i}\left[d_{i}\right] \in \Psi_{2}\left(\mathrm{SO}\left(V_{2 n}\right)\right) / \sim_{\epsilon}$ with $\Sigma_{i} \in$ $\mathcal{A}_{\text {cusp }}\left(\mathrm{GL}_{m_{i}}\right)$, we put

$$
m_{\Sigma}= \begin{cases}1 & \text { if } \Sigma \in \Psi_{2}^{\epsilon}\left(\mathrm{SO}\left(V_{2 n}\right)\right) / \sim_{\epsilon}, \text { i.e., } m_{i} d_{i} \text { is odd for some } i \\ 2 & \text { otherwise }\end{cases}
$$

and

$$
\Pi_{\Sigma}^{0}\left(\varepsilon_{\Sigma}\right)=\left\{\left[\sigma_{0}\right] \in \Pi_{\Sigma}^{0}\left|\iota_{c}\left(\left[\sigma_{0}\right]\right) \circ \Delta=\varepsilon_{\Sigma}\right| \mathcal{S}_{\Sigma}^{+}\right\} .
$$

Moreover, if $\Sigma \in \Psi_{2, \text { temp }}\left(\mathrm{SO}\left(V_{2 n}\right)\right)$, then $\varepsilon_{\Sigma}$ is the trivial representation of $\mathcal{S}_{\Sigma}$.

For the definition of $\varepsilon_{\Sigma}$, see the remark after [Ar, Theorem 1.5.2]. 
Remark 6.9. In fact, Arthur described a spectral decomposition of

$$
L_{\text {disc }}^{2}\left(\mathrm{SO}\left(V_{2 n}\right)(\mathbb{F}) \backslash \mathrm{SO}\left(V_{2 n}\right)(\mathbb{A})\right) .
$$

However it is well understood (by Harish-Chadra, Langlands, etc.) that $\mathcal{A}_{2}\left(\mathrm{SO}\left(V_{2 n}\right)\right)$ is a dense subspace of $L_{\text {disc }}^{2}\left(\mathrm{SO}\left(V_{2 n}\right)(\mathbb{F}) \backslash \mathrm{SO}\left(V_{2 n}\right)(\mathbb{A})\right)$. So we shall work with $\mathcal{A}_{2}\left(\mathrm{SO}\left(V_{2 n}\right)\right)$ in this paper.

Remark 6.10. Taïbi [Ta] proved the multiplicity formula for $\mathrm{SO}\left(V_{2 n}\right)$ when $\mathrm{SO}\left(V_{2 n}\right)\left(\mathbb{F}_{\infty}\right)$ is compact and $\mathrm{SO}\left(V_{2 n}\right)\left(\mathbb{F}_{v}\right)$ is quasi-split at all finite places $v$ of $\mathbb{F}$.

6.8. Arthur's multiplicity formula for $\mathrm{SO}\left(V_{2 n}\right)(\mathbb{A}) \cdot \mathrm{O}\left(V_{2 n}\right)(\mathbb{F})$. Theorem 6.8 follows from a more precise result. In this subsection, we recall this result.

Let $\epsilon \in \mathrm{O}\left(V_{2 n}\right)(\mathbb{F})$ be as in 6.1 . We may consider $\mathcal{H}\left(\mathrm{SO}\left(V_{2 n}\right)\right) \rtimes\langle\epsilon\rangle$, where $\epsilon$ acts on $\mathcal{H}\left(\mathrm{SO}\left(V_{2 n}\right)\right)$ by

$$
\left(\epsilon f \epsilon^{-1}\right)(x):=f\left(\epsilon^{-1} x \epsilon\right)
$$

for $f \in \mathcal{H}\left(\mathrm{SO}\left(V_{2 n}\right)\right)$. We say that $\left(\sigma, \mathcal{V}_{\sigma}\right)$ is an $\left(\mathcal{H}\left(\mathrm{SO}\left(V_{2 n}\right)\right), \epsilon\right)$-module if

- $\left(\sigma, \mathcal{V}_{\sigma}\right)$ is an $\mathcal{H}\left(\mathrm{SO}\left(V_{2 n}\right)\right)$-module;

- there is an automorphism $\sigma(\epsilon)$ on $\mathcal{V}_{\sigma}$ such that $\sigma(\epsilon)^{2}=\mathbf{1}_{\mathcal{V}_{\sigma}}$;

- $\sigma\left(\epsilon f \epsilon^{-1}\right) \circ \sigma(\epsilon)=\sigma(\epsilon) \circ \sigma(f)$ for any $f \in \mathcal{H}\left(\mathrm{SO}\left(V_{2 n}\right)\right)$.

We define an action of $\epsilon$ on $\mathcal{A}_{2}\left(\mathrm{SO}\left(V_{2 n}\right)\right)$ by

$$
(\epsilon \cdot \varphi)\left(h_{0}\right)=\varphi\left(\epsilon^{-1} h_{0} \epsilon\right) .
$$

It makes $\mathcal{A}_{2}\left(\mathrm{SO}\left(V_{2 n}\right)\right)$ an $\left(\mathcal{H}\left(\mathrm{SO}\left(V_{2 n}\right)\right), \epsilon\right)$-module.

For an $\mathcal{H}\left(\mathrm{SO}\left(V_{2 n}\right)\right)$-module $\left(\sigma, \mathcal{V}_{\sigma}\right)$, we define an $\mathcal{H}\left(\mathrm{SO}\left(V_{2 n}\right)\right)$-module $\left(\sigma^{\epsilon}, \mathcal{V}_{\sigma^{\epsilon}}\right)$ by $\mathcal{V}_{\sigma}=\mathcal{V}_{\sigma^{\epsilon}}$ and

$$
\sigma^{\epsilon}(f) v=\sigma\left(\epsilon^{-1} f \epsilon\right) v
$$

for $f \in \mathcal{H}\left(\operatorname{SO}\left(V_{2 n}\right)\right)$ and $v \in \mathcal{V}_{\sigma}$.

Lemma 6.11. If $\mathcal{V}_{0} \subset \mathcal{A}_{2}\left(\mathrm{SO}\left(V_{2 n}\right)\right)$ is an irreducible $\mathcal{H}\left(\mathrm{SO}\left(V_{2 n}\right)\right)$-summand isomorphic to $\sigma$, then the subspace

$$
\mathcal{V}_{0}^{\epsilon}=\left\{\epsilon \cdot \varphi \mid \varphi \in \mathcal{V}_{0}\right\}
$$

is an irreducible $\mathcal{H}\left(\mathrm{SO}\left(V_{2 n}\right)\right)$-summand isomorphic to $\sigma^{\epsilon}$.

Proof. Obvious.

Now, we recall a result of Arthur $\mathrm{Ar}$ which states a decomposition of $\mathcal{A}_{2}\left(\mathrm{SO}\left(V_{2 n}\right)\right)$ as an $\left(\mathcal{H}\left(\mathrm{SO}\left(V_{2 n}\right)\right), \epsilon\right)$-module by using global $A$-parameters. Let $\Sigma=\boxplus_{i=1}^{l} \Sigma_{i}\left[d_{i}\right] \in \Psi_{2}\left(\mathrm{SO}\left(V_{2 n}\right)\right) / \sim_{\epsilon}$ and $\left[\sigma_{0}\right] \in \Pi_{\Sigma}^{0}$. We take a representative $\sigma_{0}$ which occurs in $\mathcal{A}_{2}\left(\mathrm{SO}\left(V_{2 n}\right)\right)$, and we denote by $\mathcal{V}_{0}$ a subspace of $\mathcal{A}_{2}\left(\mathrm{SO}\left(V_{2 n}\right)\right)$ which realizes $\sigma_{0}$. We distinguish 3 cases as follows:

(A) Suppose that $\Sigma \in \Psi_{2}^{\epsilon}\left(\mathrm{SO}\left(V_{2 n}\right)\right) / \sim_{\epsilon}$. In this case, we have $m_{\Sigma}=1$. Hence $\mathcal{V}_{0}$ is stable under the action of $\epsilon$, and so that $\sigma_{0}^{\epsilon} \cong \sigma_{0}$. The space $\mathcal{V}_{0}$ realizes a distinguished extension of $\sigma_{0}$ to an $\left(\mathcal{H}\left(\mathrm{SO}\left(V_{2 n}\right)\right), \epsilon\right)$-module.

(B) Suppose that $\Sigma \notin \Psi_{2}^{\epsilon}\left(\mathrm{SO}\left(V_{2 n}\right)\right) / \sim_{\epsilon}$ and $\sigma_{0}^{\epsilon} \not \sigma_{0}$. In this case, $m_{\Sigma}=2$ and $\mathcal{V}_{0}^{\epsilon} ¥ \mathcal{V}_{0}$. This shows that both $\sigma_{0}$ and $\sigma_{0}^{\epsilon}$ occur in $\mathcal{A}_{2}\left(\operatorname{SO}\left(V_{2 n}\right)\right)$. This explains why $m_{\Sigma}=2$. 
(C) Suppose that $\Sigma \notin \Psi_{2}^{\epsilon}\left(\mathrm{SO}\left(V_{2 n}\right)\right) / \sim_{\epsilon}$ and $\sigma_{0}^{\epsilon} \cong \sigma_{0}$. Then there are exactly two extensions $\sigma_{1}$ and $\sigma_{2}$ of $\sigma_{0}$ to $\left(\mathcal{H}\left(\mathrm{SO}\left(V_{2 n}\right)\right), \epsilon\right)$-modules. Moreover, $\mathrm{Ar}$, Theorem 4.2.2 (a)] implies that both $\sigma_{1}$ and $\sigma_{2}$ occur in $\mathcal{A}_{2}\left(\mathrm{SO}\left(V_{2 n}\right)\right)$. This explains why $m_{\Sigma}=2$.

In case (A), there are exactly two extensions $\sigma_{1}$ and $\sigma_{2}$ of $\sigma_{0}$ to $\left(\mathcal{H}\left(\mathrm{SO}\left(V_{2 n}\right)\right), \epsilon\right)$ modules. The above argument shows that exactly one of $\sigma_{1}$ or $\sigma_{2}$ occurs in $\mathcal{A}_{2}\left(\mathrm{SO}\left(V_{2 n}\right)\right)$. The following theorem determines which extension occurs.

Theorem 6.12 ([ㅍ, Theorem 4.2.2]). Let $\Sigma \in \Psi_{2}^{\epsilon}\left(\mathrm{SO}\left(V_{2 n}\right)\right) / \sim_{\epsilon}$ and $\left[\sigma_{0}\right] \in \Pi_{\Sigma}^{0}$. Assume that an $\mathcal{H}\left(\mathrm{SO}\left(V_{2 n}\right)\right)$-module $\sigma_{0}=\otimes_{v}^{\prime} \sigma_{0, v}$ occurs in $\mathcal{A}_{2}\left(\mathrm{SO}\left(V_{2 n}\right)\right)$, so that $\iota_{c}\left(\left[\sigma_{0}\right]\right) \circ \Delta=\varepsilon_{\Sigma} \mid \mathcal{S}_{\Sigma}^{+}$by Arthur's multiplicity formula. For each place $v$ of $\mathbb{F}$, take an extension $\sigma_{v}$ of $\sigma_{0, v}$ to an $\left(\mathcal{H}\left(\mathrm{SO}\left(V_{2 n}\right)\left(\mathbb{F}_{v}\right)\right), \epsilon_{v}\right)$-module such that $\iota_{c}\left(\sigma_{v}\right)=\mathbf{1}$ for almost all $v$. Put $\sigma=\otimes_{v}^{\prime} \sigma_{v}$. Let $\iota_{c}(\sigma)$ be the character of $\prod_{v} \mathcal{S}_{\Sigma_{v}}$ defined by

$$
\iota_{c}(\sigma)=\prod_{v} \iota_{c}\left(\sigma_{v}\right)
$$

Then as an $\left(\mathcal{H}\left(\mathrm{SO}\left(V_{2 n}\right)\right), \epsilon\right)$-module, $\sigma$ occurs in $\mathcal{A}_{2}\left(\mathrm{SO}\left(V_{2 n}\right)\right)$ if and only if $\iota_{c}(\sigma) \circ$ $\Delta=\varepsilon_{\Sigma}$.

\section{Arthur's multiplicity FORMUla FOR $\mathrm{O}\left(V_{2 n}\right)$}

In this section, we prove Arthur's multiplicity formula for $\mathrm{O}\left(V_{2 n}\right)$, which is the third main theorem in this paper.

7.1. Statements. Let $\sigma=\otimes_{v}^{\prime} \sigma_{v}$ and $\sigma^{\prime}=\otimes_{v}^{\prime} \sigma_{v}^{\prime}$ be two admissible representations of $\mathcal{H}\left(\mathrm{O}\left(V_{2 n}\right)\right)$. We say that $\sigma$ and $\sigma^{\prime}$ are nearly equivalent if $\sigma_{v} \cong \sigma_{v}^{\prime}$ for almost all $v$. In this case, we write $\sigma \sim_{\text {ne }} \sigma^{\prime}$. Similarly, let $\left[\sigma_{0}\right]=\otimes_{v}^{\prime}\left[\sigma_{0, v}\right]$ and $\left[\sigma_{0}^{\prime}\right]=\otimes_{v}^{\prime}\left[\sigma_{0, v}^{\prime}\right]$ be two equivalence classes of admissible representations of $\mathcal{H}^{\epsilon}\left(\mathrm{SO}\left(V_{2 n}\right)\right)$. We say that $\left[\sigma_{0}\right]$ and $\left[\sigma_{0}^{\prime}\right]$ are $\epsilon$-nearly equivalent if $\left[\sigma_{0, v}\right]=\left[\sigma_{0, v}^{\prime}\right]$, i.e., $\sigma_{0, v}^{\prime} \cong \sigma_{0, v}$ or $\sigma_{0, v}^{\epsilon}$ for almost all $v$. In this case, we write $\left[\sigma_{0}\right] \sim_{\text {ne }}\left[\sigma_{0}^{\prime}\right]$.

By a full near equivalence class in $\mathcal{A}_{2}\left(\mathrm{O}\left(V_{2 n}\right)\right)$, we mean a maximal $\mathcal{H}\left(\mathrm{O}\left(V_{2 n}\right)\right)$ submodule $\mathcal{V}$ of $\mathcal{A}_{2}\left(\mathrm{O}\left(V_{2 n}\right)\right)$ such that

- all irreducible constituents of $\mathcal{V}$ are nearly equivalent to each other;

- any irreducible $\mathcal{H}\left(\mathrm{O}\left(V_{2 n}\right)\right)$-submodule of $\mathcal{A}_{2}\left(\mathrm{O}\left(V_{2 n}\right)\right)$ which is orthogonal to $\mathcal{V}$ is not nearly equivalent to the constituents of $\mathcal{V}$.

We define an $\epsilon$-near equivalence class in $\mathcal{A}_{2}\left(\mathrm{SO}\left(V_{2 n}\right)\right)$ similarly.

Let $\Sigma=\boxplus_{i=1}^{l} \Sigma_{i}\left[d_{i}\right] \in \Psi_{2}\left(\mathrm{O}\left(V_{2 n}\right)\right)$. For almost all places $v$ of $\mathbb{F}$, we denote the Satake parameter of $\Sigma_{i, v}$ by $c_{i, v}$, which is a semi-simple conjugacy class of $\mathrm{GL}_{m_{i}}(\mathbb{C})$. We say that a full near equivalence class $\mathcal{V}$ in $\mathcal{A}_{2}\left(\mathrm{O}\left(V_{2 n}\right)\right)$ is determined by $\Sigma$ if for any irreducible submodule $\sigma=\otimes_{v}^{\prime} \sigma_{v}$ of $\mathcal{V}$ and for almost all places $v$ of $\mathbb{F}$, the Satake parameter of $\sigma_{v}$ is given by

$$
\bigoplus_{i=1}^{l}\left(c_{i, v} \otimes\left(\begin{array}{llll}
q_{v}^{-\frac{d_{i}-1}{2}} & & & \\
& q_{v}^{-\frac{d_{i}-3}{2}} & & \\
& & \ddots & \\
& & & q_{v}^{\frac{d_{i}-1}{2}}
\end{array}\right)\right),
$$

which is a semi-simple conjugacy class of $\mathrm{O}_{2 n}(\mathbb{C})$. 
The global main theorem is Arthur's multiplicity formula for $\mathrm{O}\left(V_{2 n}\right)$, which is formulated as follows:

Theorem 7.1 (Arthur's multiplicity formula for $\mathrm{O}\left(V_{2 n}\right)$ ). $\quad$ (1) The discrete spectrum $\mathcal{A}_{2}\left(\mathrm{O}\left(V_{2 n}\right)\right)$ is a direct sum of the full near equivalence classes $\mathcal{A}_{2, \Sigma}$ determined by $\Sigma \in \Psi_{2}\left(\mathrm{O}\left(V_{2 n}\right)\right)$, i.e.,

$$
\mathcal{A}_{2}\left(\mathrm{O}\left(V_{2 n}\right)\right) \cong \bigoplus_{\Sigma \in \Psi_{2}\left(\mathrm{O}\left(V_{2 n}\right)\right)} \mathcal{A}_{2, \Sigma}
$$

(2) The restriction map Res: $\mathcal{A}\left(\mathrm{O}\left(V_{2 n}\right)\right) \rightarrow \mathcal{A}\left(\mathrm{SO}\left(V_{2 n}\right)\right)$ induces a surjective $\left(\mathcal{H}\left(\mathrm{SO}\left(V_{2 n}\right)\right), \epsilon\right)$-homomorphism Res: $\mathcal{A}_{2}\left(\mathrm{O}\left(V_{2 n}\right)\right) \rightarrow \mathcal{A}_{2}\left(\mathrm{SO}\left(V_{2 n}\right)\right)$. The submodule $\mathcal{A}_{2, \Sigma}$ is the inverse image of

$$
m_{\Sigma} \bigoplus_{\left[\sigma_{0}\right] \in \Pi_{\Sigma}^{0}\left(\varepsilon_{\Sigma}\right)}\left[\sigma_{0}\right]
$$

under this map.

(3) If $\Sigma$ is a tempered A-parameter, then we have a decomposition

$$
\mathcal{A}_{2, \Sigma} \cong \bigoplus_{\sigma \in \Pi_{\Sigma}(\mathbf{1})} \sigma
$$

as $\mathcal{H}\left(\mathrm{O}\left(V_{2 n}\right)\right)$-modules, where

$$
\Pi_{\Sigma}(\mathbf{1})=\left\{\sigma \in \Pi_{\Sigma} \mid \iota_{c}(\sigma) \circ \Delta=\mathbf{1}\right\} .
$$

(4) Assume Conjectures 6.1 and 6.6. Then

$$
\mathcal{A}_{2, \Sigma} \cong \bigoplus_{\sigma \in \Pi_{\Sigma}\left(\varepsilon_{\Sigma}\right)} \sigma
$$

where

$$
\Pi_{\Sigma}\left(\varepsilon_{\Sigma}\right)=\left\{\sigma \in \Pi_{\Sigma} \mid \iota_{c}(\sigma) \circ \Delta=\varepsilon_{\Sigma}\right\} .
$$

Also, we will show the following:

Proposition 7.2. (1) If $\Sigma \in \Psi_{2}\left(\mathrm{O}\left(V_{2 n}\right)\right)$ is tempered, then for any $\sigma \in \Pi_{\Sigma}(\mathbf{1})$, we have

$$
\operatorname{dim}_{\mathbb{C}} \operatorname{Hom}_{\mathcal{H}\left(\mathrm{O}\left(V_{2 n}\right)\right)}\left(\sigma, \mathcal{A}_{2}\left(\mathrm{O}\left(V_{2 n}\right)\right)\right)=1 .
$$

In other words, the tempered part of the automorphic discrete spectrum of $\mathrm{O}\left(V_{2 n}\right)$

$$
\mathcal{A}_{2, \text { temp }}\left(\mathrm{O}\left(V_{2 n}\right)\right):=\bigoplus_{\Sigma \in \Psi_{2, \text { temp }}\left(\mathrm{O}\left(V_{2 n}\right)\right)} \bigoplus_{\sigma \in \Pi_{\Sigma}(\mathbf{1})} \sigma
$$

is multiplicity-free as an $\mathcal{H}\left(\mathrm{O}\left(V_{2 n}\right)\right)$-module.

(2) Assume Conjectures 6.1 and 6.6. Then for an irreducible $\mathcal{H}\left(\mathrm{O}\left(V_{2 n}\right)\right)$ module $\sigma$, we have

$$
\operatorname{dim}_{\mathbb{C}} \operatorname{Hom}_{\mathcal{H}\left(\mathrm{O}\left(V_{2 n}\right)\right)}\left(\sigma, \mathcal{A}_{2}\left(\mathrm{O}\left(V_{2 n}\right)\right)\right) \leq 1 .
$$

In other words, if Conjectures 6.1 and 6.6 hold, then $\mathcal{A}_{2}\left(\mathrm{O}\left(V_{2 n}\right)\right)$ is multiplicity-free as an $\mathcal{H}\left(\mathrm{O}\left(V_{2 n}\right)\right)$-module.

Remark 7.3. As we have seen in Propositions 6.2 and 6.7 when $\mathbb{F}$ is totally complex, both of Conjectures 6.1 and 6.6 hold. Therefore we can apply Theorem 7.1 and Proposition 7.2 in this case. 
The rest of this section is devoted to the proofs of Theorem 7.1 and Proposition 7.2 .

7.2. Near equivalence classes and $A$-parameters. In this subsection, we relate $\epsilon$-near equivalence classes in $\mathcal{A}_{2}\left(\mathrm{SO}\left(V_{2 n}\right)\right)$ with elements in $\Psi_{2}\left(\mathrm{SO}\left(V_{2 n}\right)\right) / \sim_{\epsilon}$.

Proposition 7.4. There exists a canonical bijection

$\left\{\epsilon\right.$-near equivalence classes in $\left.\mathcal{A}_{2}\left(\mathrm{SO}\left(V_{2 n}\right)\right)\right\} \longleftrightarrow \Psi_{2}\left(\mathrm{SO}\left(V_{2 n}\right)\right) / \sim_{\epsilon}$.

Proof. Suppose that $\left[\sigma_{0}\right]$ and $\left[\sigma_{0}^{\prime}\right]$ appear in $\mathcal{A}_{2}\left(\mathrm{SO}\left(V_{2 n}\right)\right)$. Let $\Sigma$ and $\Sigma^{\prime}$ be $A$ parameters in $\Psi_{2}\left(\mathrm{SO}\left(V_{2 n}\right)\right) / \sim_{\epsilon}$ associated to $\left[\sigma_{0}\right]$ and $\left[\sigma_{0}^{\prime}\right]$, i.e., $\left[\sigma_{0}\right] \in \Pi_{\Sigma}^{0}$ and $\left[\sigma_{0}^{\prime}\right] \in \Pi_{\Sigma^{\prime}}^{0}$, respectively. We claim that $\left[\sigma_{0}\right] \sim_{n e}\left[\sigma_{0}^{\prime}\right]$ if and only if $\Sigma=\Sigma^{\prime}$. Suppose that $\left[\sigma_{0}\right],\left[\sigma_{0}^{\prime}\right] \in \Pi_{\Sigma}^{0}$. Note that both of $\sigma_{0, v}$ and $\sigma_{0, v}^{\prime}$ are unramified for almost all $v$. By [M1, $\$ 4.4$ Proposition], for such $v$, we have $\left[\sigma_{0, v}\right]=\left[\sigma_{0, v}^{\prime}\right]$. Hence $\left[\sigma_{0}\right] \sim_{\text {ne }}\left[\sigma_{0}^{\prime}\right]$.

Conversely, suppose that $\left[\sigma_{0}\right] \sim_{\text {ne }}\left[\sigma_{0}^{\prime}\right]$. For each place $v$ of $\mathbb{F}$, we decompose

$$
\Sigma_{v}=\left(\phi_{1, v} \otimes S_{d_{1}}\right) \oplus \cdots \oplus\left(\phi_{l, v} \otimes S_{d_{l}}\right), \quad \Sigma_{v}^{\prime}=\left(\phi_{1, v}^{\prime} \otimes S_{d_{1}^{\prime}}\right) \oplus \cdots \oplus\left(\phi_{l, v}^{\prime} \otimes S_{d_{l^{\prime}}^{\prime}}\right),
$$

where $\phi_{i, v}$ and $\phi_{j, v}^{\prime}$ are representations of $W D_{\mathbb{F}_{v}}=W_{\mathbb{F}_{v}} \times \mathrm{SL}_{2}(\mathbb{C})$. Note that for almost all $v, \phi_{i, v}$ and $\phi_{j, v}^{\prime}$ are trivial on $\mathrm{SL}_{2}(\mathbb{C})$. Hence by [M1, $\S 4.2$ Corollaire], we have $\Sigma_{v} \cong \Sigma_{v}^{\prime}$ for almost all $v$. As in $\mathrm{Ar}$, §1.3], $\Sigma$ and $\Sigma^{\prime}$ define isobaric sums of representations $\phi_{\Sigma}$ and $\phi_{\Sigma^{\prime}}$ which belong to the discrete spectrum of GL(2n). It follows by the generalized strong multiplicity 1 theorem of Jacquet-Shalika ([JS2, (4.4) Theorem]) that $\phi_{\Sigma} \cong \phi_{\Sigma^{\prime}}$. Since the map $\Sigma \mapsto \phi_{\Sigma}$ is injective, we conclude that $\Sigma=\Sigma^{\prime}$.

Corollary 7.5. Let $\Sigma, \Sigma^{\prime} \in \Psi_{2}\left(\mathrm{SO}\left(V_{2 n}\right)\right) / \sim_{\epsilon}$. If $\Sigma \neq \Sigma^{\prime}$, then $\Pi_{\Sigma}^{0} \cap \Pi_{\Sigma^{\prime}}^{0}=\emptyset$.

This corollary together with Arthur's multiplicity formula (Theorem 6.8) and the argument in 6.8 gives the multiplicity in $\mathcal{A}_{2}\left(\mathrm{SO}\left(V_{2 n}\right)\right)$.

Corollary 7.6. Assume Conjectures 6.1 and 6.6.

(1) For $\left[\sigma_{0}\right] \in \Pi_{\Sigma}^{0}$, we have

$$
\operatorname{dim}_{\mathbb{C}} \operatorname{Hom}_{\mathcal{H}^{\epsilon}\left(\operatorname{SO}\left(V_{2 n}\right)\right)}\left(\left[\sigma_{0}\right], \mathcal{A}_{2}\left(\operatorname{SO}\left(V_{2 n}\right)\right)\right)= \begin{cases}m_{\Sigma} & \text { if } \iota_{c}\left(\left[\sigma_{0}\right]\right)=\varepsilon_{\Sigma}, \\ 0 & \text { otherwise. }\end{cases}
$$

(2) For any irreducible $\left(\mathcal{H}\left(\mathrm{SO}\left(V_{2 n}\right)\right), \epsilon\right)$-module $\sigma$, we have

$$
\operatorname{dim}_{\mathbb{C}} \operatorname{Hom}_{\left(\mathcal{H}\left(\mathrm{SO}\left(V_{2 n}\right)\right), \epsilon\right)}\left(\sigma, \mathcal{A}_{2}\left(\mathrm{SO}\left(V_{2 n}\right)\right)\right) \leq 1 .
$$

In other words, $\mathcal{A}_{2}\left(\mathrm{SO}\left(V_{2 n}\right)\right)$ is multiplicity-free as an $\left(\mathcal{H}\left(\mathrm{SO}\left(V_{2 n}\right)\right), \epsilon\right)$ module.

In particular, the same properties hold unconditionally for the tempered part of the automorphic discrete spectrum of $\mathrm{SO}\left(V_{2 n}\right)$

$$
\mathcal{A}_{2, \text { temp }}\left(\mathrm{SO}\left(V_{2 n}\right)\right):=\bigoplus_{\Sigma \in \Psi_{2, \text { temp }}\left(\operatorname{SO}\left(V_{2 n}\right)\right) / \sim_{\epsilon}\left[\sigma_{0}\right] \in \Pi_{\Sigma}^{0}\left(\varepsilon_{\Sigma}\right)} m_{\Sigma}\left[\sigma_{0}\right] .
$$

Proof. By Arthur's multiplicity formula (Theorem 6.8), we have

$$
\mathcal{A}_{2}\left(\mathrm{SO}\left(V_{2 n}\right)\right) \cong \bigoplus_{\Sigma \in \Psi_{2}\left(\operatorname{SO}\left(V_{2 n}\right)\right) / \sim_{\epsilon}\left[\sigma_{0}\right] \in \Pi_{\Sigma}^{0}\left(\varepsilon_{\Sigma}\right)} m_{\Sigma}\left[\sigma_{0}\right]
$$


as $\mathcal{H}^{\epsilon}\left(\mathrm{SO}\left(V_{2 n}\right)\right)$-modules. Since $\Pi_{\Sigma}^{0} \cap \Pi_{\Sigma^{\prime}}^{0}=\emptyset$ for $\Sigma \neq \Sigma^{\prime}$ by Corollary 7.5 , and $\Pi_{\Sigma}^{0}$ is a multiplicity-free set by Conjectures 6.1 and 6.6 , we have

$$
\operatorname{dim}_{\mathbb{C}} \operatorname{Hom}_{\mathcal{H}^{\epsilon}\left(\operatorname{SO}\left(V_{2 n}\right)\right)}\left(\left[\sigma_{0}\right], \mathcal{A}_{2}\left(\operatorname{SO}\left(V_{2 n}\right)\right)\right)= \begin{cases}m_{\Sigma} & \text { if } \iota_{c}\left(\left[\sigma_{0}\right]\right)=\varepsilon_{\Sigma} \\ 0 & \text { otherwise }\end{cases}
$$

This is (1). The proof of (2) is similar. See also the arguments (A), (B) and (C) in 6.8

7.3. Restriction of automorphic forms. Now we compare $\mathcal{A}_{2}\left(\mathrm{O}\left(V_{2 n}\right)\right)$ with $\mathcal{A}_{2}\left(\mathrm{SO}\left(V_{2 n}\right)\right)$. To do this, we consider the restriction map

$$
\text { Res: } \mathcal{A}\left(\mathrm{O}\left(V_{2 n}\right)\right) \rightarrow \mathcal{A}\left(\mathrm{SO}\left(V_{2 n}\right)\right), \varphi \mapsto \varphi \mid \mathrm{SO}\left(V_{2 n}\right)(\mathbb{A}) \text {. }
$$

Lemma 7.7. We have $\operatorname{Res}\left(\mathcal{A}_{2}\left(\mathrm{O}\left(V_{2 n}\right)\right)\right) \subset \mathcal{A}_{2}\left(\mathrm{SO}\left(V_{2 n}\right)\right)$.

Proof. Let $\varphi \in \mathcal{A}_{2}\left(\mathrm{O}\left(V_{2 n}\right)\right)$. Then

$$
\int_{\mathrm{O}\left(V_{2 n}\right)(\mathbb{F}) \backslash \mathrm{O}\left(V_{2 n}\right)(\mathbb{A})}|\varphi(g)|^{2} d g=\int_{\mu_{2}(\mathbb{F}) \backslash \mu_{2}(\mathbb{A})}\left(\int_{\mathrm{SO}\left(V_{2 n}\right)(\mathbb{F}) \backslash \mathrm{SO}\left(V_{2 n}\right)(\mathbb{A})}\left|\varphi\left(h \epsilon_{t}\right)\right|^{2} d h\right) d t
$$

is finite. By Fubini's theorem, we see that for almost every $t \in \mu_{2}(\mathbb{F}) \backslash \mu_{2}(\mathbb{A})$ (with respect to the Haar measure of $\left.\mu_{2}(\mathbb{F}) \backslash \mu_{2}(\mathbb{A})\right)$, the function $h \mapsto\left|\varphi\left(h \epsilon_{t}\right)\right|^{2}$ is integrable on $\mathrm{SO}\left(V_{2 n}\right)(\mathbb{F}) \backslash \mathrm{SO}\left(V_{2 n}\right)(\mathbb{A})$.

Since $\varphi(g)$ is right $K$-finite, there exists a finite set $S$ of finite places of $\mathbb{F}$ containing all infinite places of $\mathbb{F}$ such that $\varphi$ is right $\epsilon_{t}$-invariant for any $t \in \prod_{v \notin S} \mu_{2}\left(\mathbb{F}_{v}\right)$. Since $\mu_{2}\left(\mathbb{F}_{\infty}\right)$ is finite, we see that $\prod_{v \notin S} \mu_{2}\left(\mathbb{F}_{v}\right)$ is open in $\mu_{2}(\mathbb{A})$ (not only in $\left.\mu_{2}\left(\mathbb{A}_{\text {fin }}\right)\right)$. This implies that

$$
\int_{\mathrm{SO}\left(V_{2 n}\right)(\mathbb{F}) \backslash \mathrm{SO}\left(V_{2 n}\right)(\mathbb{A})}\left|\varphi\left(h \epsilon_{t}\right)\right|^{2} d h<\infty
$$

for some (hence any) $t \in \prod_{v \notin S} \mu_{2}\left(\mathbb{F}_{v}\right)$. In particular, $\operatorname{Res}(\varphi)$ is square-integrable.

Proposition 7.8. We have $\operatorname{Res}\left(\mathcal{A}_{2}\left(\mathrm{O}\left(V_{2 n}\right)\right)\right)=\mathcal{A}_{2}\left(\mathrm{SO}\left(V_{2 n}\right)\right)$.

Proof. Let $\varphi_{0} \in \mathcal{A}_{2}\left(\mathrm{SO}\left(V_{2 n}\right)\right)$. Since $\varphi_{0}$ is $K_{0}$-finite, there exists a compact open subgroup $K_{1}$ of $K_{0} \cap \mathrm{SO}\left(V_{2 n}\right)\left(\mathbb{A}_{\text {fin }}\right)$ such that $\varphi_{0}$ is right $K_{1}$-invariant. We may assume that $K_{1}$ is of the form $K_{1}=\prod_{v<\infty} K_{1, v}$ for some compact open subgroup $K_{1, v}$ of $K_{0, v}$ such that $\epsilon_{v}^{-1} K_{1, v} \epsilon_{v}=K_{1, v}$ for any $v<\infty$. Moreover, we can find a finite set $S$ of places of $\mathbb{F}$ containing all infinite places of $\mathbb{F}$ such that $K_{1, v}=K_{0, v}$ for any $v \notin S$. We fix a complete system $B$ of representatives of

$$
\mu_{2}(\mathbb{F}) \backslash\left(\prod_{v \in S} \mu_{2}\left(\mathbb{F}_{v}\right)\right) .
$$

We may assume that $B$ contains the identity element $1 \in \prod_{v \in S} \mu_{2}\left(\mathbb{F}_{v}\right)$.

We regard $\varphi_{0}$ as a function on $\mathrm{O}\left(V_{2 n}\right)(\mathbb{F}) \cdot \mathrm{SO}\left(V_{2 n}\right)(\mathbb{A})$, which is left $\mathrm{O}\left(V_{2 n}\right)(\mathbb{F})$ invariant. For $t \in \mu_{2}(\mathbb{A})$, we define a function $\varphi_{t}: \mathrm{O}\left(V_{2 n}\right)(\mathbb{F}) \cdot \mathrm{SO}\left(V_{2 n}\right)(\mathbb{A}) \rightarrow \mathbb{C}$ by

$$
\varphi_{t}(h)= \begin{cases}\varphi_{0}(h) & \text { if }\left(t_{v}\right)_{v \in S} \in B \\ \varphi_{0}(h \epsilon) & \text { if }\left(t_{v}\right)_{v \in S} \notin B\end{cases}
$$


for $h \in \mathrm{O}\left(V_{2 n}\right)(\mathbb{F}) \cdot \mathrm{SO}\left(V_{2 n}\right)(\mathbb{A})$. Then we see that

$$
\varphi_{t a}(h)=\varphi_{t}(h), \quad \varphi_{-t}(h)=\varphi_{t}(h \epsilon)
$$

for $t \in \mu_{2}(\mathbb{A})$ and $a \in \prod_{v \notin S} \mu_{2}\left(\mathbb{F}_{v}\right)$ since $\epsilon^{2}=\mathbf{1}_{V_{2 n}}$. Moreover, $\varphi_{t}$ is right $K_{1^{-}}$ invariant for any $t \in \mu_{2}(\mathbb{A})$.

Now we define a function $\varphi: \mathrm{O}\left(V_{2 n}\right)(\mathbb{A}) \rightarrow \mathbb{C}$ by

$$
\varphi(g)=\varphi_{\operatorname{det}(g)}\left(g \epsilon_{\operatorname{det}(g)}^{-1}\right)
$$

for $g \in \mathrm{O}\left(V_{2 n}\right)(\mathbb{A})$. Then we have $\operatorname{Res}(\varphi)=\varphi_{0}$. We show that $\varphi \in \mathcal{A}_{2}\left(\mathrm{O}\left(V_{2 n}\right)\right)$.

Let $\gamma \in \mathrm{O}\left(V_{2 n}\right)(\mathbb{F})$. If $\operatorname{det}(\gamma)=1$, we have

$$
\varphi(\gamma g)=\varphi_{\operatorname{det}(g)}\left(\gamma g \epsilon_{\operatorname{det}(g)}^{-1}\right)=\varphi_{\operatorname{det}(g)}\left(g \epsilon_{\operatorname{det}(g)}^{-1}\right)=\varphi(g) .
$$

If $\operatorname{det}(\gamma)=-1$, we have

$$
\varphi(\gamma g)=\varphi_{-\operatorname{det}(g)}\left(\gamma g \epsilon_{-\operatorname{det}(g)}^{-1}\right)=\varphi_{\operatorname{det}(g)}\left(g \epsilon_{-\operatorname{det}(g)}^{-1} \epsilon\right)=\varphi(g) .
$$

Hence $\varphi$ is left $\mathrm{O}\left(V_{2 n}\right)(\mathbb{F})$-invariant. It is easy to see that $\varphi$ is right $\left(K_{1} \cdot \prod_{v \notin S} K_{v}\right)$ invariant and is a $C^{\infty}$-function on $\mathrm{O}\left(V_{2 n}\right)\left(\mathbb{F}_{\infty}\right)$. Hence $\varphi$ is a smooth function on $\mathrm{O}\left(V_{2 n}\right)(\mathbb{A})$.

We denote the space spanned by $k \cdot \varphi$ for $k \in K$ (resp. for $k \in K_{0}$ ) by $K \varphi$ (resp. $\left.K_{0} \varphi\right)$. Since any $\varphi^{\prime} \in K \varphi$ is right $\prod_{v \notin S} K_{v}$-invariant, the finiteness of $\operatorname{dim}(K \varphi)$ is equivalent to that of $\operatorname{dim}\left(K_{0} \varphi\right)$. So we shall prove that $\operatorname{dim}\left(K_{0} \varphi\right)<\infty$. Let

$$
\varphi^{\prime}=\sum_{i=1}^{r} c_{i}\left(k_{i} \cdot \varphi\right) \in K_{0} \varphi
$$

with $c_{i} \in \mathbb{C}$ and $k_{i} \in K_{0}$. Then for $a \in \prod_{v \in S} \mu_{2}\left(\mathbb{F}_{v}\right)$ and $x \in \operatorname{SO}\left(V_{2 n}\right)(\mathbb{A})$, we have

$$
\left(\epsilon_{a} \cdot \varphi^{\prime}\right)(x)=\varphi^{\prime}\left(x \epsilon_{a}\right)=\sum_{i=1}^{r} c_{i} \varphi\left(x \epsilon_{a} k_{i}\right)=\sum_{i=1}^{r} c_{i} \varphi\left(x\left(\epsilon_{a} k_{i} \epsilon_{a}^{-1}\right) \epsilon_{a}\right) .
$$

Since $\epsilon_{a} k_{i} \epsilon_{a}^{-1} \in K_{0}$, we have

$$
\left(\epsilon_{a} \cdot \varphi^{\prime}\right) \mid \mathrm{SO}\left(V_{2 n}\right)(\mathbb{A}) \in K_{0}\left(\left(\epsilon_{a} \cdot \varphi\right) \mid \mathrm{SO}\left(V_{2 n}\right)(\mathbb{A})\right) .
$$

Hence we may consider the map

$$
\Phi: K_{0} \varphi \rightarrow \bigoplus_{a} K_{0}\left(\left(\epsilon_{a} \cdot \varphi\right) \mid \mathrm{SO}\left(V_{2 n}\right)(\mathbb{A})\right), \varphi^{\prime} \mapsto \oplus_{a}\left(\left(\epsilon_{a} \cdot \varphi^{\prime}\right) \mid \operatorname{SO}\left(V_{2 n}\right)(\mathbb{A})\right)_{a},
$$

where $a$ runs over $\prod_{v \in S} \mu_{2}\left(\mathbb{F}_{v}\right)$. Since any $\varphi^{\prime} \in K_{0} \varphi$ is right $\prod_{v \notin S} K_{v}$-invariant and the map

$$
\prod_{v \in S} \mu_{2}\left(\mathbb{F}_{v}\right) \rightarrow \mathrm{SO}\left(V_{2 n}\right)(\mathbb{A}) \backslash \mathrm{O}\left(V_{2 n}\right)(\mathbb{A}) / \prod_{v \notin S} K_{v}, a \mapsto \epsilon_{a}
$$

is bijective, we see that $\Phi$ is injective. Since $\left(\epsilon_{a} \cdot \varphi^{\prime}\right) \mid \operatorname{SO}\left(V_{2 n}\right)(\mathbb{A}) \in \mathcal{A}\left(\operatorname{SO}\left(V_{2 n}\right)\right)$, it is $K_{0}$-finite. Hence we have $\operatorname{dim}\left(K_{0} \varphi\right)<\infty$, and so we get the $K$-finiteness of $\varphi$. Similarly, we obtain the $\mathfrak{z}$-finiteness of $\varphi$. Note that $\operatorname{Lie}\left(\mathrm{O}\left(V_{2 n}\right)\left(\mathbb{F}_{\infty}\right)\right)=$ $\operatorname{Lie}\left(\operatorname{SO}\left(V_{2 n}\right)\left(\mathbb{F}_{\infty}\right)\right)$.

Now we show that $\|\varphi\|_{L^{2}\left(\mathrm{O}\left(V_{2 n}\right)\right)}<\infty$, where $\|\cdot\|_{L^{2}\left(\mathrm{O}\left(V_{2 n}\right)\right)}\left(\right.$ resp. $\left.\|\cdot\|_{L^{2}\left(\mathrm{SO}\left(V_{2 n}\right)\right)}\right)$ is the $L^{2}$-norm on $\mathrm{O}\left(V_{2 n}\right)(\mathbb{F}) \backslash \mathrm{O}\left(V_{2 n}\right)(\mathbb{A})\left(\right.$ resp. $\left.\mathrm{SO}\left(V_{2 n}\right)(\mathbb{F}) \backslash \mathrm{SO}\left(V_{2 n}\right)(\mathbb{A})\right)$. Let $\Omega$ be 
the characteristic function on $\prod_{v \notin S} \mu_{2}\left(\mathbb{F}_{v}\right) \times B \subset \mu_{2}(\mathbb{A})$. Then we have

$$
\begin{aligned}
\|\varphi\|_{L^{2}\left(\mathrm{O}\left(V_{2 n}\right)\right)}^{2} & =\int_{\mathrm{O}\left(V_{2 n}\right)(\mathbb{F}) \backslash \mathrm{O}\left(V_{2 n}\right)(\mathbb{A})}|\varphi(g)|^{2} d g \\
& =\int_{\mu_{2}(\mathbb{F}) \backslash \mu_{2}(\mathbb{A})}\left(\int_{\mathrm{SO}\left(V_{2 n}\right)(\mathbb{F}) \backslash \mathrm{SO}\left(V_{2 n}\right)(\mathbb{A})}\left|\varphi\left(h \epsilon_{t}\right)\right|^{2} d h\right) d t \\
& =\int_{\mu_{2}(\mathbb{A})} \Omega(t) \cdot\left(\int_{\mathrm{SO}\left(V_{2 n}\right)(\mathbb{F}) \backslash \mathrm{SO}\left(V_{2 n}\right)(\mathbb{A})}\left|\varphi\left(h \epsilon_{t}\right)\right|^{2} d h\right) d t \\
& =\int_{\mu_{2}(\mathbb{A})} \Omega(t) \cdot\left(\int_{\mathrm{SO}\left(V_{2 n}\right)(\mathbb{F}) \backslash \mathrm{SO}\left(V_{2 n}\right)(\mathbb{A})}\left|\varphi_{0}(h)\right|^{2} d h\right) d t \\
& \left.=\operatorname{vol}\left(\prod_{v \notin S} \mu_{2}\left(\mathbb{F}_{v}\right) \times B\right) \cdot\left\|\varphi_{0}\right\|_{L^{2}\left(\mathrm{SO}\left(V_{2 n}\right)\right)}^{2}\right) \\
& =2^{-1}\left\|\varphi_{0}\right\|_{L^{2}\left(\mathrm{SO}\left(V_{2 n}\right)\right)}^{2}<\infty .
\end{aligned}
$$

We have shown that $\varphi$ is a smooth, $K$-finite, $\mathfrak{z}$-finite, square-integrable function on $\mathrm{O}\left(V_{2 n}\right)(\mathbb{F}) \backslash \mathrm{O}\left(V_{2 n}\right)(\mathbb{A})$. Such functions are of moderate growth (see [BJ, §4.3]). Therefore we conclude that $\varphi \in \mathcal{A}_{2}\left(\mathrm{O}\left(V_{2 n}\right)\right)$. This completes the proof.

Note that Res: $\mathcal{A}_{2}\left(\mathrm{O}\left(V_{2 n}\right)\right) \rightarrow \mathcal{A}_{2}\left(\mathrm{SO}\left(V_{2 n}\right)\right)$ is an $\left(\mathcal{H}\left(\mathrm{SO}\left(V_{2 n}\right)\right), \epsilon\right)$-homomorphism. Proposition 7.8 says that this homomorphism is surjective. Also we need the following lemma.

Lemma 7.9. Assume Conjectures 6.1 and 6.6. Let $\sigma$ be an irreducible $\mathcal{H}\left(\mathrm{O}\left(V_{2 n}\right)\right)$ submodule of $\mathcal{A}_{2}\left(\mathrm{O}\left(V_{2 n}\right)\right)$. Then $\operatorname{Res}(\sigma)$ is non-zero and irreducible as an $\left(\mathcal{H}\left(\mathrm{SO}\left(V_{2 n}\right)\right), \epsilon\right)$-module.

Proof. It is clear that $\operatorname{Res}(\sigma)$ is non-zero. Decompose $\sigma \cong \otimes_{v}^{\prime} \sigma_{v}$. If $\sigma_{v} \otimes \operatorname{det} \approx \sigma_{v}$ for any $v$, then $\sigma$ is irreducible as an $\left(\mathcal{H}\left(\mathrm{SO}\left(V_{2 n}\right)\right), \epsilon\right)$-module. Hence so is $\operatorname{Res}(\sigma)$.

We may assume that $\sigma_{v} \otimes \operatorname{det} \cong \sigma_{v}$ for some $v$. This is in the case (B) as in 6.8 We decompose

$$
\sigma \cong \bigoplus_{\lambda \in \Lambda} \sigma_{\lambda}
$$

into a direct sum of irreducible $\left(\mathcal{H}\left(\mathrm{SO}\left(V_{2 n}\right)\right), \epsilon\right)$-modules. Here, $\Lambda$ is an index set. Then $\operatorname{Res}(\sigma) \cong \oplus_{\lambda \in \Lambda_{0}} \sigma_{\lambda}$ for some non-empty subset $\Lambda_{0}$ of $\Lambda$. As an $\mathcal{H}^{\epsilon}\left(\mathrm{SO}\left(V_{2 n}\right)\right)$ module, each $\sigma_{\lambda}$ is a direct sum of two copies of an irreducible $\mathcal{H}^{\epsilon}\left(\mathrm{SO}\left(V_{2 n}\right)\right)$-module $\left[\sigma_{0}\right]$. Hence $\operatorname{Res}(\sigma) \cong\left[\sigma_{0}\right]^{\oplus 2\left|\Lambda_{0}\right|}$ as $\mathcal{H}^{\epsilon}\left(\mathrm{SO}\left(V_{2 n}\right)\right)$-modules. By Corollary 7.6 (which we have shown using Conjectures 6.1 and (6.6), we have

$$
\operatorname{dim}_{\mathbb{C}} \operatorname{Hom}_{\mathcal{H}^{\epsilon}\left(\mathrm{SO}\left(V_{2 n}\right)\right)}\left(\left[\sigma_{0}\right], \mathcal{A}_{2}\left(\mathrm{SO}\left(V_{2 n}\right)\right)\right) \leq 2 .
$$

This implies that $\left|\Lambda_{0}\right|=1$. Hence $\operatorname{Res}(\sigma)$ is irreducible as an $\left(\mathcal{H}\left(\operatorname{SO}\left(V_{2 n}\right)\right), \epsilon\right)$ module.

Remark 7.10. For an irreducible $(\mathcal{H}(\mathrm{SO}(2 n)), \epsilon)$-submodule $\sigma_{0}$ of $\mathcal{A}_{2}\left(\mathrm{SO}\left(V_{2 n}\right)\right)$, the induction

is defined by the space of functions

$$
\operatorname{Ind}_{\mathrm{O}\left(V_{2 n}\right)(\mathbb{F}) \cdot \mathrm{SO}\left(V_{2 n}\right)(\mathbb{A})}^{\mathrm{O}\left(V_{2 n}\right)\left(\sigma_{0}\right)}
$$

$$
\Phi_{*}: \mathrm{O}\left(V_{2 n}\right)(\mathbb{A}) \rightarrow \sigma_{0}, g \mapsto \Phi_{g}
$$

such that 
- $\Phi_{h g}(x)=\Phi_{g}(x h)$ for $h \in \mathrm{O}\left(V_{2 n}\right)(\mathbb{F}) \cdot \mathrm{SO}\left(V_{2 n}\right)(\mathbb{A}), g \in \mathrm{O}\left(V_{2 n}\right)(\mathbb{A})$ and $x \in \mathrm{SO}\left(V_{2 n}\right)(\mathbb{A})$

- for each $x \in \operatorname{SO}\left(V_{2 n}\right)(\mathbb{A})$, the function $g \mapsto \Phi_{g}(x) \in \mathbb{C}$ is a smooth function on $\mathrm{O}\left(V_{2 n}\right)(\mathbb{A})$.

The Hecke algebra $\mathcal{H}\left(\mathrm{O}\left(V_{2 n}\right)\right)$ acts on $\Phi_{*}$ by

$$
\left(f \cdot \Phi_{*}\right)_{g}(x)=\int_{\mathrm{O}\left(V_{2 n}\right)(\mathbb{A})} f(y) \Phi_{g y}(x) d y
$$

for $f \in \mathcal{H}\left(\mathrm{O}\left(V_{2 n}\right)\right)$. Then by (the proof of) Proposition 7.8 together with Lemma 7.9, we see that the map $\Phi_{*} \mapsto\left[g \mapsto \Phi_{g}(1)\right]$ gives an $\mathcal{H}\left(\mathrm{O}\left(V_{2 n}\right)\right)$-isomorphism

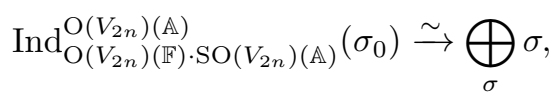

where $\sigma$ runs over the set of irreducible $\mathcal{H}\left(\mathrm{O}\left(V_{2 n}\right)\right)$-submodules $\sigma$ of $\mathcal{A}_{2}\left(\mathrm{O}\left(V_{2 n}\right)\right)$ such that $\operatorname{Res}(\sigma)=\sigma_{0}$.

Now we relate the near equivalence classes in $\mathcal{A}_{2}\left(\mathrm{O}\left(V_{2 n}\right)\right)$ with the $\epsilon$-near equivalence classes in $\mathcal{A}_{2}\left(\mathrm{SO}\left(V_{2 n}\right)\right)$.

Proposition 7.11. The map Res: $\mathcal{A}_{2}\left(\mathrm{O}\left(V_{2 n}\right)\right) \rightarrow \mathcal{A}_{2}\left(\mathrm{SO}\left(V_{2 n}\right)\right)$ induces a bijection \{near equivalence classes in $\left.\mathcal{A}_{2}\left(\mathrm{O}\left(V_{2 n}\right)\right)\right\}$

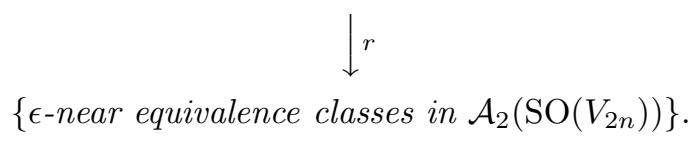

Proof. Let $\Phi$ be a near equivalence class in $\mathcal{A}_{2}\left(\mathrm{O}\left(V_{2 n}\right)\right)$. Then $\operatorname{Res}(\Phi)$ will a priori meet several $\epsilon$-near equivalence classes. Suppose that for an irreducible $\mathcal{H}\left(\mathrm{O}\left(V_{2 n}\right)\right)$ module $\sigma$ belonging to $\Phi, \operatorname{Res}(\sigma)$ contains an irreducible $\mathcal{H}\left(\operatorname{SO}\left(V_{2 n}\right)\right)$-module $\sigma_{0}$ and $\left[\sigma_{0}\right]$ belongs to an $\epsilon$-near equivalence class $\Phi_{0}$. Then we claim that

$$
\operatorname{Res}(\Phi) \subset \Phi_{0} .
$$

Indeed, suppose that $\sigma^{\prime} \in \Phi, \operatorname{Res}\left(\sigma^{\prime}\right) \supset \sigma_{0}^{\prime}$, and $\left[\sigma_{0}^{\prime}\right]$ belongs to an $\epsilon$-near equivalence class $\Phi_{0}^{\prime}$. Then for almost all $v$, we have $\sigma_{v}^{\prime} \cong \sigma_{v}$ so that $\sigma_{0, v}^{\prime} \cong \sigma_{0, v}$ or $\sigma_{0, v}^{\epsilon}$. This means that $\left[\sigma_{0}\right] \sim_{\text {ne }}\left[\sigma_{0}^{\prime}\right]$ so that $\Phi_{0}=\Phi_{0}^{\prime}$. Therefore if we define $r(\Phi)=\Phi_{0}$, then $r$ is well-defined.

For the injectivity, if $r(\Phi)=r\left(\Phi^{\prime}\right)$, then for $\sigma \in \Phi$ and $\sigma^{\prime} \in \Phi^{\prime}$, one has $\sigma_{v}^{\prime} \cong \sigma_{v}$ or $\sigma_{v}^{\prime} \otimes \operatorname{det}$ for almost all $v$. Since $\sigma_{v}$ and $\sigma_{v}^{\prime}$ are unramified with respect to $K_{v}$ for almost all $v$, we must have $\sigma_{v}^{\prime} \cong \sigma_{v}$ for almost all $v$ (see Lemma 2.4). Hence we have $\Phi=\Phi^{\prime}$.

For the surjectivity, we decompose

$$
\mathcal{A}_{2}\left(\mathrm{O}\left(V_{2 n}\right)\right) \cong \bigoplus_{\lambda} \sigma_{\lambda}
$$

into a direct sum of irreducible $\mathcal{H}\left(\mathrm{O}\left(V_{2 n}\right)\right)$-modules. Then we may decompose

$$
\sigma_{\lambda} \cong \bigoplus_{\kappa} \sigma_{\lambda, \kappa}
$$

into a direct sum of irreducible $\mathcal{H}\left(\mathrm{SO}\left(V_{2 n}\right)\right)$-modules. Since Res: $\mathcal{A}_{2}\left(\mathrm{O}\left(V_{2 n}\right)\right) \rightarrow$ $\mathcal{A}_{2}\left(\mathrm{SO}\left(V_{2 n}\right)\right)$ is a surjective $\mathcal{H}\left(\mathrm{SO}\left(V_{2 n}\right)\right)$-homomorphism, any irreducible $\mathcal{H}\left(\mathrm{SO}\left(V_{2 n}\right)\right)$-submodule $\sigma_{0}$ of $\mathcal{A}_{2}\left(\mathrm{SO}\left(V_{2 n}\right)\right)$ is isomorphic to some $\sigma_{\lambda, \kappa}$ via Res. 
Then we have $\operatorname{Res}\left(\sigma_{\lambda}\right) \supset \sigma_{0}$. This shows that If $\Phi_{0}\left(\operatorname{resp} . \Phi_{\lambda}\right)$ is the $\epsilon$-near equivalence class of $\sigma_{0}$ (resp. the near equivalence class of $\sigma_{\lambda}$ ), then $r\left(\Phi_{\lambda}\right)=\Phi_{0}$.

7.4. Proof of Theorem [7.1. In this subsection, we will complete the proof of Theorem 7.1 and show Proposition 7.2

Recall that $\Psi_{2}\left(\mathrm{O}\left(V_{2 n}\right)\right)=\Psi_{2}\left(\mathrm{SO}\left(V_{2 n}\right)\right) / \sim_{\epsilon}$. By Propositions 7.4 and 7.11 , we obtain a canonical bijection

$$
\text { \{near equivalence classes in } \left.\mathcal{A}_{2}\left(\mathrm{O}\left(V_{2 n}\right)\right)\right\} \longleftrightarrow \Psi_{2}\left(\mathrm{O}\left(V_{2 n}\right)\right) \text {. }
$$

In other words, we obtain a decomposition

$$
\mathcal{A}_{2}\left(\mathrm{O}\left(V_{2 n}\right)\right)=\bigoplus_{\Sigma \in \Psi_{2}\left(\mathrm{O}\left(V_{2 n}\right)\right)} \mathcal{A}_{2, \Sigma}
$$

where $\mathcal{A}_{2, \Sigma}$ is the direct sum over the near equivalence class corresponding to $\Sigma$. By the construction, $\mathcal{A}_{2, \Sigma}$ is the inverse image of

$$
m_{\Sigma} \bigoplus_{\left[\sigma_{0}\right] \in \Pi_{\Sigma}^{0}\left(\varepsilon_{\Sigma}\right)}\left[\sigma_{0}\right]
$$

under Res: $\mathcal{A}_{2}\left(\mathrm{O}\left(V_{2 n}\right)\right) \rightarrow \mathcal{A}_{2}\left(\mathrm{SO}\left(V_{2 n}\right)\right)$. Moreover, we have

$$
\mathcal{A}_{2, \Sigma}=\bigoplus_{\sigma \in \Pi_{\Sigma}} m(\sigma) \sigma
$$

for some $m(\sigma) \in \mathbb{Z}_{\geq 0}$. We have to show that

$$
m(\sigma)= \begin{cases}1 & \text { if } \iota_{c}(\sigma) \circ \Delta=\varepsilon_{\Sigma}, \\ 0 & \text { otherwise. }\end{cases}
$$

Consider the restriction map Res: $\mathcal{A}_{2}\left(\mathrm{O}\left(V_{2 n}\right)\right) \rightarrow \mathcal{A}_{2}\left(\mathrm{SO}\left(V_{2 n}\right)\right)$. This is an $\left(\mathcal{H}\left(\mathrm{SO}\left(V_{2 n}\right)\right), \epsilon\right)$-equivariant homomorphism. By Arthur's multiplicity formulas for $\mathrm{SO}\left(V_{2 n}\right)$ and $\mathrm{SO}\left(V_{2 n}\right)(\mathbb{A}) \cdot \mathrm{O}\left(V_{2 n}\right)(\mathbb{F})$ (Theorems 6.8 and 6.12), we see that if $\sigma \in \Pi_{\Sigma}$ occurs in $\mathcal{A}_{2}\left(\mathrm{O}\left(V_{2 n}\right)\right)$, then $\operatorname{Res}(\sigma) \neq 0$ so that $\iota_{c}(\sigma) \circ \Delta=\varepsilon_{\Sigma}$. In other words, if $\sigma \in \Pi_{\Sigma}$ satisfies $\iota_{c}(\sigma) \circ \Delta \neq \varepsilon_{\Sigma}$, then $\sigma$ does not occur in $\mathcal{A}_{2}\left(\mathrm{O}\left(V_{2 n}\right)\right)$, i.e., $m(\sigma)=0$.

Now we consider the case when $\iota_{c}(\sigma) \circ \Delta=\varepsilon_{\Sigma}$.

Proposition 7.12. Assume Conjectures 6.1] and 6.6. Let $\sigma=\otimes_{v} \sigma_{v} \in \Pi_{\Sigma}$ such that $\iota_{c}(\sigma) \circ \Delta=\varepsilon_{\Sigma}$. Then there exists an $\mathcal{H}\left(\mathrm{O}\left(V_{2 n}\right)\right)$-subspace $\mathcal{A}_{\sigma}$ of $\mathcal{A}_{2}\left(\mathrm{O}\left(V_{2 n}\right)\right)$ on which $\mathcal{H}\left(\mathrm{O}\left(V_{2 n}\right)\right)$ acts by $\sigma$.

Proof. Let $\left[\sigma_{0}\right] \in \Pi_{\Sigma}^{0}$ be an element satisfying $\sigma_{0, v} \subset \sigma_{v} \mid \mathrm{SO}\left(V_{2 n}\right)\left(\mathbb{F}_{v}\right)$ for each $v$. By Arthur's multiplicity formula (Theorem 6.8), we see that $\left[\sigma_{0}\right]$ occurs in $\mathcal{A}_{2}\left(\mathrm{SO}\left(V_{2 n}\right)\right.$ ) as an $\mathcal{H}^{\epsilon}\left(\mathrm{SO}\left(V_{2 n}\right)\right)$-module. We may assume that $\sigma_{0}$ occurs in $\mathcal{A}_{2}\left(\mathrm{SO}\left(V_{2 n}\right)\right)$ as an $\mathcal{H}\left(\mathrm{SO}\left(V_{2 n}\right)\right)$-module. Since Res: $\mathcal{A}_{2}\left(\mathrm{O}\left(V_{2 n}\right)\right) \rightarrow \mathcal{A}_{2}\left(\mathrm{SO}\left(V_{2 n}\right)\right)$ is surjective (Proposition (7.8), and $\mathcal{A}_{2}\left(\mathrm{O}\left(V_{2 n}\right)\right)$ is a direct sum of irreducible $\mathcal{H}\left(\mathrm{O}\left(V_{2 n}\right)\right)$-modules, we can find an irreducible $\mathcal{H}\left(\mathrm{O}\left(V_{2 n}\right)\right)$-module $\sigma^{\prime}=\otimes_{v} \sigma_{v}^{\prime}$ such that $\sigma^{\prime}$ occurs in $\mathcal{A}_{2}\left(\mathrm{O}\left(V_{2 n}\right)\right)$ and $\operatorname{Res}\left(\sigma^{\prime}\right) \supset \sigma_{0}$. By (the proof of) Proposition 7.11, we see that $\sigma^{\prime} \in \Pi_{\Sigma}$ and

$$
\sigma^{\prime} \cong \sigma \otimes \operatorname{det}_{S}
$$

for some finite set $S$ of places of $\mathbb{F}$. Here, $\operatorname{det}_{S}$ is the determinant for places in $S$ and trivial outside $S$. We consider 3 cases (A), (B) and (C) as in 6.8 separately. 
We consider the case (A). Suppose that $\Sigma \in \Psi_{2}^{\epsilon}\left(\mathrm{O}\left(V_{2 n}\right)\right)$. Then $\left[\mathcal{S}_{\Sigma}: \mathcal{S}_{\Sigma}^{+}\right]=2$ and $\left[\mathcal{S}_{\Sigma, v}: \mathcal{S}_{\Sigma, v}^{+}\right]=2$ for any place $v$ of $\mathbb{F}$. By Conjectures 6.1 and 6.6, we see that

$$
\iota_{c}\left(\sigma^{\prime}\right)=\iota_{c}(\sigma) \otimes\left(\prod_{v \in S} \eta_{0, v}\right),
$$

where $\eta_{0, v}$ is the non-trivial character of $\mathcal{S}_{\Sigma, v} / \mathcal{S}_{\Sigma, v}^{+}$. Hence we have

$$
\iota_{c}\left(\sigma^{\prime}\right) \circ \Delta=\left(\iota_{c}(\sigma) \circ \Delta\right) \cdot \eta_{0}^{|S|}=\epsilon_{\Sigma} \cdot \eta_{0}^{|S|},
$$

where $\eta_{0}$ is the non-trivial character of $\mathcal{S}_{\Sigma} / \mathcal{S}_{\Sigma}^{+}$. Since $\operatorname{Res}\left(\sigma^{\prime}\right)$ occurs in $\mathcal{A}_{2}\left(\operatorname{SO}\left(V_{2 n}\right)\right)$ as an $\left(\mathcal{H}\left(\mathrm{SO}\left(V_{2 n}\right)\right), \epsilon\right)$-module, by Theorem 6.12, the number of $S$ must be even. Hence if $\varphi^{\prime} \in \mathcal{A}_{2}\left(\mathrm{O}\left(V_{2 n}\right)\right)$, then

$$
\varphi(g):=\operatorname{det}_{S}(g) \cdot \varphi^{\prime}(g) \in \mathcal{A}_{2}\left(\mathrm{O}\left(V_{2 n}\right)\right) .
$$

This implies that $\sigma$ also occurs in $\mathcal{A}_{2}\left(\mathrm{O}\left(V_{2 n}\right)\right)$.

We consider the case (B). Suppose that $\Sigma \notin \Psi_{2}^{\epsilon}\left(\mathrm{O}\left(V_{2 n}\right)\right)$ and $\sigma_{0}^{\epsilon} \neq \sigma_{0}$. Then $\sigma_{v} \otimes \operatorname{det}_{v} \cong \sigma_{v}$ for some $v$. Hence we can take $S$ such that $|S|$ is even, and we see that $\sigma$ also occurs in $\mathcal{A}_{2}\left(\mathrm{O}\left(V_{2 n}\right)\right)$.

We consider the case (C). Suppose that $\Sigma \notin \Psi_{2}^{\epsilon}\left(\mathrm{O}\left(V_{2 n}\right)\right)$ and $\sigma_{0}^{\epsilon} \cong \sigma_{0}$. Then there are two extensions of $\sigma_{0}$ to irreducible $\left(\mathcal{H}\left(\mathrm{SO}\left(V_{2 n}\right)\right), \epsilon\right)$-modules, and both of them occur in $\mathcal{A}_{2}\left(\mathrm{SO}\left(V_{2 n}\right)\right)$. Note that $\operatorname{Res}\left(\sigma^{\prime}\right) \cong \operatorname{Res}\left(\sigma^{\prime} \otimes \operatorname{det}_{S}\right)$ as $\left(\mathcal{H}\left(\mathrm{SO}\left(V_{2 n}\right)\right), \epsilon\right)$ modules if and only if $|S|$ is even. This implies that $\sigma \cong \sigma^{\prime} \otimes \operatorname{det}_{S}$ occurs in $\mathcal{A}_{2}\left(\mathrm{O}\left(V_{2 n}\right)\right)$ for any $S$. This completes the proof.

Under assuming Conjectures 6.1 and 6.6, by a similar argument to Corollary 7.6 , we have

$$
m(\sigma)=\operatorname{dim}_{\mathbb{C}} \operatorname{Hom}_{\mathcal{H}\left(\mathrm{O}\left(V_{2 n}\right)\right)}\left(\sigma, \mathcal{A}_{2}\left(\mathrm{O}\left(V_{2 n}\right)\right)\right) .
$$

Hence Proposition 7.12 says that if $\iota_{c}(\sigma) \circ \Delta=\varepsilon_{\Sigma}$, then $m(\sigma)>0$. To prove $m(\sigma)=1$ for such $\sigma$, it suffices to show Proposition 7.2 .

Proof of Proposition 7.2. Let $\sigma$ be an irreducible $\mathcal{H}\left(\mathrm{O}\left(V_{2 n}\right)\right)$-module. We have to show that

$$
\operatorname{dim}_{\mathbb{C}} \operatorname{Hom}_{\mathcal{H}\left(\mathrm{O}\left(V_{2 n}\right)\right)}\left(\sigma, \mathcal{A}_{2}\left(\mathrm{O}\left(V_{2 n}\right)\right)\right) \leq 1 .
$$

Suppose for the sake of contradiction that $\sigma$ is an irreducible $\mathcal{H}\left(\mathrm{O}\left(V_{2 n}\right)\right)$-module such that

$$
\operatorname{dim}_{\mathbb{C}} \operatorname{Hom}_{\mathcal{H}\left(\mathrm{O}\left(V_{2 n}\right)\right)}\left(\sigma, \mathcal{A}_{2}\left(\mathrm{O}\left(V_{2 n}\right)\right)\right) \geq 2 .
$$

We know that as an $\left(\mathcal{H}\left(\mathrm{SO}\left(V_{2 n}\right)\right), \epsilon\right)$-module, $\sigma$ is a multiplicity-free sum

$$
\sigma=\bigoplus_{\lambda} \sigma_{\lambda}
$$

of irreducible $\left(\mathcal{H}\left(\mathrm{SO}\left(V_{2 n}\right)\right), \epsilon\right)$-modules $\sigma_{\lambda}$. For a fixed non-zero $f_{1}$ in the above Hom space, we have shown that $\operatorname{Res}\left(f_{1}(\sigma)\right)$ is irreducible (Lemma 7.9), so say $\operatorname{Res}\left(f_{1}(\sigma)\right)=\sigma_{\lambda_{1}}$. Consider the natural map

$$
\operatorname{Hom}_{\mathcal{H}\left(\mathrm{O}\left(V_{2 n}\right)\right)}\left(\sigma, \mathcal{A}_{2}\left(\mathrm{O}\left(V_{2 n}\right)\right)\right) \rightarrow \operatorname{Hom}_{\left(\mathcal{H}\left(\mathrm{SO}\left(V_{2 n}\right)\right), \epsilon\right)}\left(\sigma_{\lambda_{1}}, \mathcal{A}_{2}\left(\mathrm{SO}\left(V_{2 n}\right)\right)\right)
$$

given by

$$
f \mapsto \operatorname{Res} \circ f \circ \iota_{1},
$$

where $\iota_{1}: \sigma_{\lambda_{1}} \hookrightarrow \sigma$ is a fixed inclusion. We have shown that the right hand side has dimension 1 (Corollary $7.6(2)$ ), so this map has non-zero kernel, i.e., there is $f_{2}$ in the left hand side such that $0 \neq \operatorname{Res}\left(f_{2}(\sigma)\right)=\sigma_{\lambda_{2}}$, with $\lambda_{1} \neq \lambda_{2}$. This shows 
that $\mathcal{A}_{2}\left(\mathrm{SO}\left(V_{2 n}\right)\right)$ contains $\sigma_{\lambda_{1}} \oplus \sigma_{\lambda_{2}}$ as an $\left(\mathcal{H}\left(\mathrm{SO}\left(V_{2 n}\right)\right), \epsilon\right)$-module. Let $\left[\sigma_{0}\right]$ be the irreducible $\mathcal{H}^{\epsilon}\left(\mathrm{SO}\left(V_{2 n}\right)\right)$-submodule of $\sigma$. Then the above argument implies that

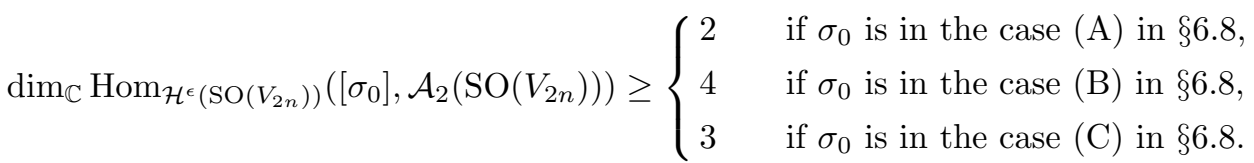

Here, in the case (C), we use the fact that $\sigma \otimes \operatorname{det}_{v}$ occurs in $\mathcal{A}_{2}\left(\mathrm{O}\left(V_{2 n}\right)\right)$ for any place $v$ of $\mathbb{F}$. This contradicts Corollary 7.6 (1). This completes the proof.

By Propositions 7.2 and 7.12 , we see that if $\iota_{c}(\sigma) \circ \Delta=\varepsilon_{\Sigma}$, then $m(\sigma)=1$. This completes the proof of Theorem 7.1 ,

\section{ACKNOWLEDGMENTS}

The authors are grateful to Professor Atsushi Ichino for his helpful comments and in particular for bringing [Ar, Theorem 2.2.4] to their attention. We also thank Dipendra Prasad for telling us his results ([P3, P4]). The first author was supported by JSPS KAKENHI Grant Number 26-1322. The second author was partially supported by a Singapore government MOE Tier 2 grant R-146-000-175112. The paper was completed when the second author visited Kyoto University in December 2015 as Distinguished Visiting Project Professor (Japan Gateway: Kyoto University Top Global Program) of Center for the Promotion of Interdisciplinary Education and Research. The second author would like to thank Atsushi Ichino for his kind invitation and Kyoto University for its generous support.

\section{REFERENCES}

[AGRS] Avraham Aizenbud, Dmitry Gourevitch, Stephen Rallis, and Gérard Schiffmann, Multiplicity one theorems, Ann. of Math. (2) 172 (2010), no. 2, 1407-1434, DOI 10.4007/annals.2010.172.1413. MR2680495

[AMR] N. Arancibia, C. Moeglin, and D. Renard, Paquets d'Arthur des groupes classiques et unitaires, arXiv:1507.01432v2.

[Ar] James Arthur, The endoscopic classification of representations, American Mathematical Society Colloquium Publications, vol. 61, American Mathematical Society, Providence, RI, 2013. Orthogonal and symplectic groups. MR3135650

[At1] H. Atobe, The local theta correspondence and the local Gan-Gross-Prasad conjecture for the symplectic-metaplectic case, arXiv:1502.03528v3.

[At2] H. Atobe, On the uniqueness of generic representations in an L-packet, Int. Math. Res. Not. IMRN, doi: 10.1093/imrn/rnw220.

[AG] H. Atobe and W. T. Gan, Local Theta correspondence of Tempered Representations and Langlands parameters, Invent. math. doi: 10.1007/s00222-017-0730-8.

[BJ] A. Borel and H. Jacquet, Automorphic forms and automorphic representations, Automorphic forms, representations and $L$-functions (Proc. Sympos. Pure Math., Oregon State Univ., Corvallis, Ore., 1977), Proc. Sympos. Pure Math., XXXIII, Amer. Math. Soc., Providence, R.I., 1979, pp. 189-207. With a supplement "On the notion of an automorphic representation" by R. P. Langlands. MR546598

[CL1] Pierre-Henri Chaudouard and Gérard Laumon, Le lemme fondamental pondéré. I. Constructions géométriques (French, with English and French summaries), Compos. Math. 146 (2010), no. 6, 1416-1506, DOI 10.1112/S0010437X10004756. MR2735371

[CL2] Pierre-Henri Chaudouard and Gérard Laumon, Le lemme fondamental pondéré. II. Énoncés cohomologiques (French, with French summary), Ann. of Math. (2) 176 (2012), no. 3, 1647-1781, DOI 10.4007/annals.2012.176.3.6. MR2979859

[CS] W. Casselman and J. Shalika, The unramified principal series of p-adic groups. II. The Whittaker function, Compositio Math. 41 (1980), no. 2, 207-231. MR.581582 
[GGP] Wee Teck Gan, Benedict H. Gross, and Dipendra Prasad, Symplectic local root numbers, central critical $L$ values, and restriction problems in the representation theory of classical groups (English, with English and French summaries), Astérisque 346 (2012), 1-109. Sur les conjectures de Gross et Prasad. I. MR 3202556

[GI1] Wee Teck Gan and Atsushi Ichino, Formal degrees and local theta correspondence, Invent. Math. 195 (2014), no. 3, 509-672, DOI 10.1007/s00222-013-0460-5. MR3166215

[GI2] Wee Teck Gan and Atsushi Ichino, The Gross-Prasad conjecture and local theta correspondence, Invent. Math. 206 (2016), no. 3, 705-799, DOI 10.1007/s00222-016-0662-8. MR3573972

[GP] Benedict H. Gross and Dipendra Prasad, On the decomposition of a representation of $\mathrm{SO}_{n}$ when restricted to $\mathrm{SO}_{n-1}$, Canad. J. Math. 44 (1992), no. 5, 974-1002, DOI 10.4153/CJM-1992-060-8. MR 1186476

[GS] Wee Teck Gan and Gordan Savin, Representations of metaplectic groups I: epsilon dichotomy and local Langlands correspondence, Compos. Math. 148 (2012), no. 6, 16551694, DOI 10.1112/S0010437X12000486. MR2999299

[GT1] Wee Teck Gan and Shuichiro Takeda, On the Howe duality conjecture in classical theta correspondence, Advances in the theory of automorphic forms and their $L$-functions, Contemp. Math., vol. 664, Amer. Math. Soc., Providence, RI, 2016, pp. 105-117, DOI 10.1090/conm/664/13063. MR3502978

[GT2] Wee Teck Gan and Shuichiro Takeda, A proof of the Howe duality conjecture, J. Amer. Math. Soc. 29 (2016), no. 2, 473-493, DOI 10.1090/jams/839. MR.3454380

$[\mathrm{H}] \quad$ Volker Heiermann, A note on standard modules and Vogan L-packets, Manuscripta Math. 150 (2016), no. 3-4, 571-583, DOI 10.1007/s00229-016-0824-4. MR3514747

[JS] H. Jacquet and J. A. Shalika, On Euler products and the classification of automorphic representations. I, Amer. J. Math. 103 (1981), no. 3, 499-558, DOI 10.2307/2374103. MR618323

[Ka1] Tasho Kaletha, Genericity and contragredience in the local Langlands correspondence, Algebra Number Theory 7 (2013), no. 10, 2447-2474, DOI 10.2140/ant.2013.7.2447. MR 3194648

[Ka2] Tasho Kaletha, Rigid inner forms of real and p-adic groups, Ann. of Math. (2) 184 (2016), no. 2, 559-632, DOI 10.4007/annals.2016.184.2.6. MR3548533

[KMSW] T. Kaletha, A. Minguez, S. W. Shin, and P.-J. White, Endoscopic classification of representations: Inner forms of unitary groups, arXiv:1409.3731v3.

[Ku] Stephen S. Kudla, On the local theta-correspondence, Invent. Math. 83 (1986), no. 2, 229-255, DOI 10.1007/BF01388961. MR.818351

[LR] Erez M. Lapid and Stephen Rallis, On the local factors of representations of classical groups, Automorphic representations, $L$-functions and applications: progress and prospects, Ohio State Univ. Math. Res. Inst. Publ., vol. 11, de Gruyter, Berlin, 2005, pp. 309-359, DOI 10.1515/9783110892703.309. MR2192828

[M1] Colette Mœglin, Comparaison des paramètres de Langlands et des exposants à l'intérieur d'un paquet d'Arthur (French, with English summary), J. Lie Theory 19 (2009), no. 4, 797-840. MR2599005

[M2] C. Mœglin, Multiplicité 1 dans les paquets d'Arthur aux places p-adiques (French, with English summary), On certain L-functions, Clay Math. Proc., vol. 13, Amer. Math. Soc., Providence, RI, 2011, pp. 333-374. MR2767522

[M3] C. Møglin, Image des opérateurs d'entrelacements normalisés et pôles des séries d'Eisenstein (French, with English and French summaries), Adv. Math. 228 (2011), no. 2, 1068-1134, DOI 10.1016/j.aim.2011.06.003. MR2822218

[MR1] C. Møglin and D. Renard, Paquets d'Arthur des groups classiques complexes, preprint, available at: https://webusers.imj-prg.fr/ colette.moeglin/GC.pdf

[MR2] C. Mœglin and D. Renard, Sur les paquets d'Arthur des groupes classiques et unitaires non quasi-déployés, preprint, available at: https://webusers.imj-prg.fr/ colette.moeglin/courtenote.pdf

[MVW] Colette Mœglin, Marie-France Vignéras, and Jean-Loup Waldspurger, Correspondances de Howe sur un corps p-adique (French), Lecture Notes in Mathematics, vol. 1291, Springer-Verlag, Berlin, 1987. MR1041060 
[MW1] Colette Mœglin and Jean-Loup Waldspurger, La conjecture locale de Gross-Prasad pour les groupes spéciaux orthogonaux: le cas général (French), Astérisque 347 (2012), 167216. Sur les conjectures de Gross et Prasad. II. MR.3155346

[MW2] C. Møglin and J.-L. Waldspurger, Stabilisation de la formule des traces tordue, Progress in Mathematics, Vol. 316/317, Birkhäuser/Springer, 2017.

[P1] Dipendra Prasad, Trilinear forms for representations of GL(2) and local $\epsilon$-factors, Compositio Math. 75 (1990), no. 1, 1-46. MR.1059954

[P2] Dipendra Prasad, On the local Howe duality correspondence, Internat. Math. Res. Notices 11 (1993), 279-287, DOI 10.1155/S1073792893000315. MR1248702

[P3] Dipendra Prasad, Some applications of seesaw duality to branching laws, Math. Ann. 304 (1996), no. 1, 1-20, DOI 10.1007/BF01446282. MR.1367880

[P4] Dipendra Prasad, Relating invariant linear form and local epsilon factors via global methods, Duke Math. J. 138 (2007), no. 2, 233-261, DOI 10.1215/S0012-7094-07-138237. With an appendix by Hiroshi Saito. MR2318284

[RS] Zeév Rudnick and Peter Sarnak, Zeros of principal L-functions and random matrix theory, Duke Math. J. 81 (1996), no. 2, 269-322, DOI 10.1215/S0012-7094-96-08115-6. A celebration of John F. Nash, Jr. MR.1395406

[S1] Freydoon Shahidi, On certain L-functions, Amer. J. Math. 103 (1981), no. 2, 297-355, DOI 10.2307/2374219. MR610479

[S2] Freydoon Shahidi, A proof of Langlands' conjecture on Plancherel measures; complementary series for p-adic groups, Ann. of Math. (2) 132 (1990), no. 2, 273-330, DOI 10.2307/1971524. MR:1070599

[Ta] O. Taïbi, Arthur's multiplicity formula for certain inner forms of special orthogonal and symplectic groups, Journal of the European Mathematical Society, to appear.

[T] J. Tate, Number theoretic background, Automorphic forms, representations and $L$ functions (Proc. Sympos. Pure Math., Oregon State Univ., Corvallis, Ore., 1977), Proc. Sympos. Pure Math., XXXIII, Amer. Math. Soc., Providence, R.I., 1979, pp. 3-26. MR.546607

[V] David A. Vogan Jr., The local Langlands conjecture, Representation theory of groups and algebras, Contemp. Math., vol. 145, Amer. Math. Soc., Providence, RI, 1993, pp. 305379, DOI 10.1090/conm/145/1216197. MR1216197

[W1] J.-L. Waldspurger, Démonstration d'une conjecture de dualité de Howe dans le cas padique, $p \neq 2$ (French), Festschrift in honor of I. I. Piatetski-Shapiro on the occasion of his sixtieth birthday, Part I (Ramat Aviv, 1989), Israel Math. Conf. Proc., vol. 2, Weizmann, Jerusalem, 1990, pp. 267-324. MR.1159105

[W2] J.-L. Waldspurger, Une formule intégrale reliée à la conjecture locale de Gross-Prasad (French, with English summary), Compos. Math. 146 (2010), no. 5, 1180-1290, DOI 10.1112/S0010437X10004744. MR2684300

[W3] Jean-Loup Waldspurger, Une formule intégrale reliée à la conjecture locale de GrossPrasad, 2e partie: extension aux représentations tempérées (French, with English and French summaries), Astérisque 346 (2012), 171-312. Sur les conjectures de Gross et Prasad. I. MR 3202558

[W4] Jean-Loup Waldspurger, Une variante d'un résultat de Aizenbud, Gourevitch, Rallis et Schiffmann (French, with English and French summaries), Astérisque 346 (2012), 313-318. Sur les conjectures de Gross et Prasad. I. MR 3202559

[W5] Jean-Loup Waldspurger, Calcul d'une valeur d'un facteur $\epsilon$ par une formule intégrale (French), Astérisque 347 (2012), 1-102. Sur les conjectures de Gross et Prasad. II. MR3155344

[W6] Jean-Loup Waldspurger, La conjecture locale de Gross-Prasad pour les représentations tempérées des groupes spéciaux orthogonaux (French), Astérisque 347 (2012), 103-165. Sur les conjectures de Gross et Prasad. II. MR3155345

[X] Bin Xu, On Moglin's Parametrization of Arthur Packets for p-adic Quasisplit $S p(N)$ and $S O(N)$, Canad. J. Math. 69 (2017), no. 4, 890-960, DOI 10.4153/CJM-2016-029-3. MR 3679701 
Department of mathematics, Kyoto University, Kitashirakawa-Oiwake-Cho, SakyoKU, КYOTO, 606-8502, JAPAN

E-mail address: atobe@math.kyoto-u.ac.jp

Department of Mathematics, National University of Singapore, 10 Lower Kent Ridge RoAD, Singapore 119076

E-mail address: matgwt@nus.edu.sg 